\title{
Bo0 8
}

\section{BODDAERT}

M. DAUBENTON 
(7) The dates of publication seem not to have been stated on the cover of the cahiers, but they can be estimated fairly exactly with the help of review's that anoeared from time to time in the 'Journal des Sçavans'.

The following reviews of the 'Planches enlumines' were nublished in the 'Journal des Sçavans' (edition in quatro):-

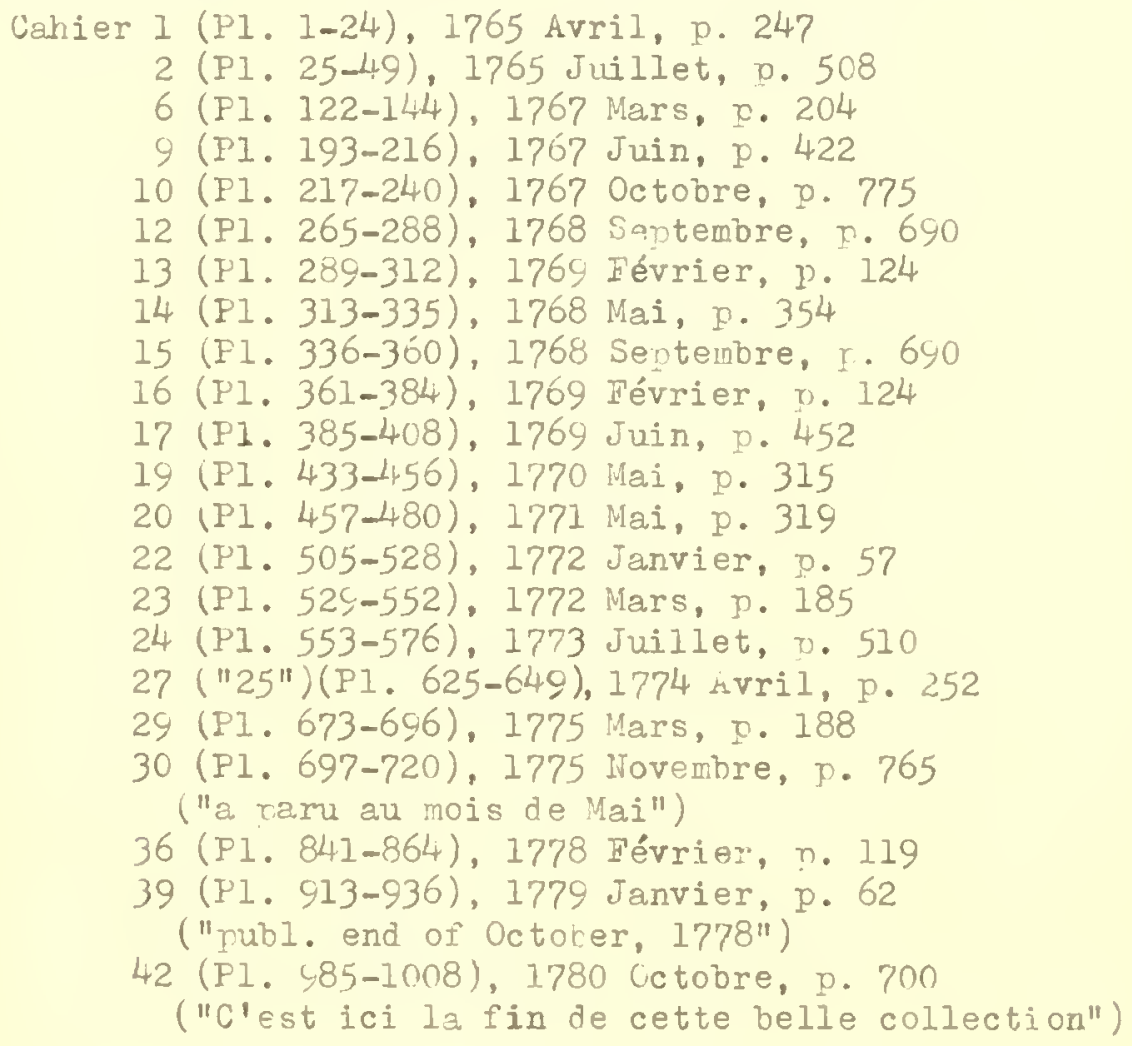

"On the Birds Collected by Pierre Poivre in Canton, Manila, India and Madagiscar (1751-1756)." by Erwin Stresemann Ibis, 94 (1952) m. 522-523. 
H 





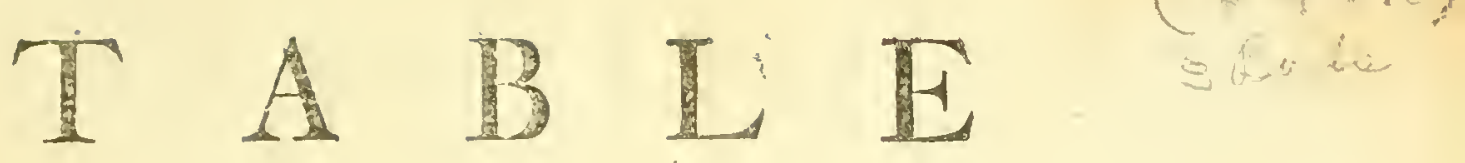

$D E S$

PLANCHES ENLUMINEEZ D'HISTOIRE NATURELLE,

D E

\section{DPAUBENTON.}

Avec les denominations de

M. M. DE BUFFON, BRISSON, EDWARDS, LINNEUS FT LATHAM.

precedé dinne Notice des Principaux Ouvrages

Zoulogiques enluminés.

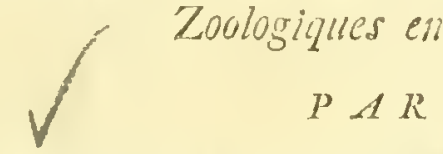

MT. BODDAEIRT, MED. Docr.

Ancien Confeiller de la wille de FLESSINGUE, membre del' Academie Imperiale de NEURNBERG, de la Societé des Sciences d' HARLEM, de VLISSIRGUE, d'U. TRECHT, de la Societé des Scrutateurs de la Natyre de BERLIN, de HALLE, se de la Societé Apiaire de TOBERLAUSNITZ.

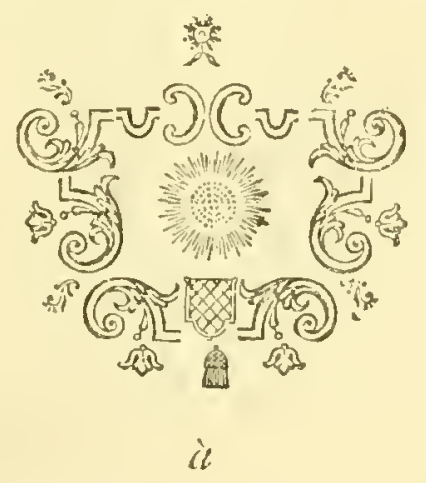

UTREGHT MIDCCLXXXII. 



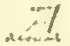

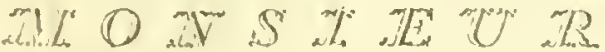 \\ LE MARQUIS DE SAINT SIMON.}

NIENBRE DE PLUSIEURS SOCIETÉS SÇAVANTES.

$(1$ 'eft a vous Monfieur, que je prends la Liberté d'offirir cé petit Effay de mes Occupations litteraires, lacceuil favorable donc vous avez bien voulu recevoir ma Theoric du Globe; ma en. hardi de vous prefenter encor cette Table des Planches enluminćes comme une marque faiblc en verité mais fincere, de m’a re. connaisfance pour toutes les politesfes, done vous ne cesfez de me comblici, Mecene éelairé des Sciences \&r des Arts, vous mavez toujours ouvert votre Bibliotheque ausf ample qu'infruetive, vos lumieres mont guidés fouvent dans le chemin te. nebreux de la Nature; fouvent encor je me rapclle les entrediens fçavants, dans les quels vous me montrais cette grande chaine, ces rapports des Etrès; que vous envifagiés d'un oeil Philofophe. Daignez done accepter cet Ouvrage avec ce front feréin, avec le quel vous regardés toujours tout ce quion vous offre pour lavancoment des 'Sciences des Arts des MIoeurs \& tout ce qui peut contribuer au bonheur de la Socicté, daignez pasler favoraeblment des defauts du Language et du ftilc vous fcavés Monfieur, que le Français n'eft pas ma Langue matemelle; fi vous y trouvez des Phrafes pasfables ou bonnes, je vous les dois, vos écrits vos discourrs inftruetits, ont étés alors les fources ou je les ai puisfé.

Si ce faible effity a le bonheur de vous plaire; Jespere de Monseur vous offrir dans l'Année fuivante, un Onrage plus etendu, fur lOn nithologic ou je raslemble, tout ce M. M. LE CoMte de Brtpfon, Camper, Spallanzani, Bloch, Silberaslach, Realmer, Hateer, Malphigi, Linnć ont donnés fur la Histoire Naturello des Oifeaux en general. 


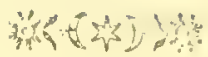

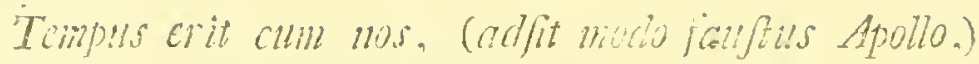

Rité Junn dosijs olinn dicentus bororem.

Tum quoque Sanct Simon nofro celibrabere Cantu,

si mudo non refugis, mang sideberis illis.

Vivéz cependant longtems cn lage a cette charmante Campagne; avee votre illuftre Epoufe; que ros travaux Champetres; foyez couromnez par les dons les plus riches donc Flore \& $P_{0}$ mone comblent leurs cultivateurs habiles; quïls foyent fouvent accompagnés, de ces travaux litteruires qui vous ont acquis deja des I.ouanges fi bien meritées; quel Amour conjugal \& l'Amitié, les deux plus grands bonheurs, dont les mortels puiflent jouir, \& que les Grands comnaisfent fi peu, remplisfent vos heures de loifir; vivés ainfi Monfieur encore une longue fuite d"Années pour votre propre bonheur, pour celui de MADAme, pour la Societé . pour l'encouragement des Arts des Sciences \& pour wos Amis, cntre lesquels, vos politesíes envers moi me donnent la hardiesfe de me compter et done la Continuation me fera toujours chere et respectable.

Je fuis

Monsieuk!

UTRECHT.

ce I Decembre $1 \% 83$.
Votre trèr bumble Es très obeiffout Serviteur

B O D A E R T.

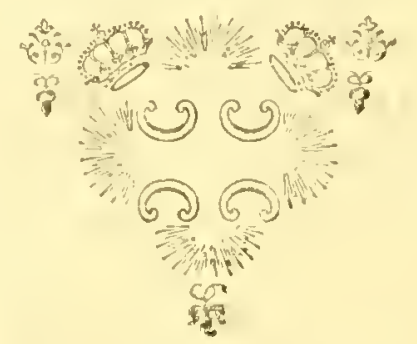

PR E. 

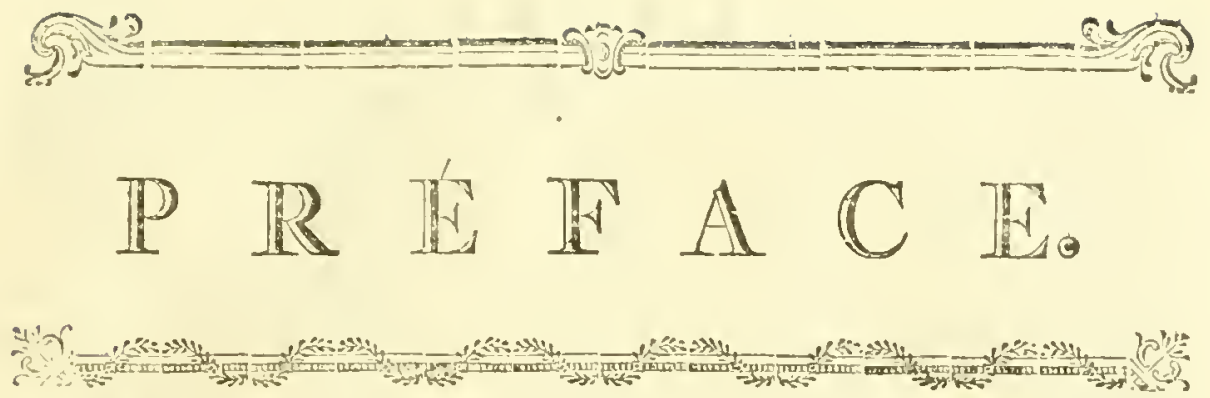

Rur narance plus, IEtule de l'Hiftoire Naturelle, que la Contetuplation des objets; c'elt la, comme M. DE Bufrox a remarqué, le premier point et la bafe de nos Connaisfances, mais puisqu'il eft imposfible de voir touts les objets en Nature, que la defeription la plus étenduc, ne nous fearroit donner des Idées claires de la Figure, de la Couleur et autres attributs des chofes, les Arts viennent au tecours de la Seience, et le Desfcin et la Gravure nous reprefentent les Contours et les parties des Animaux, des Plantes et des Mineraux, mais il refte alors encor une difficulté, la Couleur; qui ne feaurait être rendue, ni par la Grarate noire, ni par la Defuription la plus exacte; parce que nous n'arous pas des nons pour les teintes, pour beancoup des Couleurs du troifieme ranģ, et mûme les denominations de cenx du premier et fécond rang font fouvent vagues, nous nommons ce belles Couleurs, que le 1jpecire folaire nous pretente par le moyen du l'risme, Rouge, Orange, Goune, Verd, Blu, Indigo V'iolez, mais queltes divilions ne peut on fairc de chaque Coulcur; qui quoique fimple et du premier ordre, diferre neannoins d'une autre, le haut ou l'extremité fuperieure, du fpectre, elt d'un rouge Conleur de carmin, a]ris vient l'écarlate, le vernillon, qui fe melant au faune forme ces diverfes teintes mesfotcintes et muaces, qui fe perdent en Jaune et ainfi des autres; Je crois pourtant, que pour les Couleurs premieres la desterité de M. d'Aubenton en poumait venil au bout, de la méme maniere, qu'il à agi avec les picres precicufes; qu'on divife par exemple le rouge qui contient 45. parties des 3 ro. du fpectre, en dinutres partics aliquotes, par excmple en 3. 5. 9. 15 . et y comparant les Penles des plus belles Fleurs, et Fruits, et slors ou pourroit nomner cette Coulenr d'un nom nouveau on d'un nombre; pour les Coulcurs fecoudaires ou melús de deux Coulcurs prinitives; ou pourroit encore les comprer a des fleurs ou des Infectes contus; mais quoique ceja fervit d'un grand fecours, ou ne fiuroir avoir avec precition les Couleurs du troticme rangs; ou celles, qui font produites par le melange de trois Couleurs qu'eft ce par exemple le luteus color, le telaceus, le coomuleus, le glaucus, le pultus, le furmos, done Mr. Pabricius, Linveres, ete Scopour, ont decrits les Infectes? Le triangle de M. Marer, la Pyranide de M. Lagtenstein, la roue de M. PoD., font très beaux dans la Theoric, mais très difficiles a mettre en pratique par la differente gravité fpecifique des masfes des Couleurs, qu'on employe, mais je quitte cette maticre, qui m’a entrainée plus loin, que je m'ctais propofé.

Le meilleur moyen de connaitre les objets eft de les voir en Nature ou eq Granture enluminée; et dans le liecle dans le quel nous vivons, les beaux Arts montrent combien elles peuvent embellir les Sciences; donnons done un cotp d'oeil fur les ouvrages d'Hilloire Naturelle des Aninuux qui meritent G'être confideréz comme des echaircisfements à Wiiltoire Naturelle. 
Le premier ourtage, gue je connais dans ce genue eft cèi de GordonirT Alutanorphofis et Hiforia Naturalis de Jifecits ditediub. 166?. I2Fo. 3 vol. avec des ligures, cet ourrage quoigue partunt de la main di"u u Peintre, n'a d'nutres merices, que la defuription des metamorphofes de quelques Infecies, et ditre un des premiers dans ce Genre; Car la planart des fignres, font très manraifes, et beancoup font inal enluminées; M. Lifter a clonné un ecition, qui niclt jas meilleure.

Environ ce même tems Josstox domnit fon Hiforia animalim in Folio Francf. 1649. avec beancotip des planches, fort mediocres.

En I6\%1. M. PERRAult clommit fes memoires putur l'Hifnire Naturelle des nnimaux, Fol. arec des bonnes figures; ce livre lut traduit en Nllemand in 4to. en 1:53. par 21. IIUTH.

En 1:-70. M. N!Eyer publiait un hel omrrage in Folio en Allemand fous le

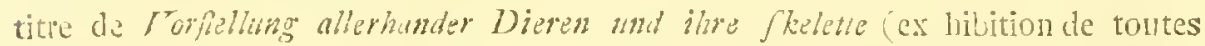
foutes d'animaux et leut liquelctes) les planches font bomnes et bien enluninées.

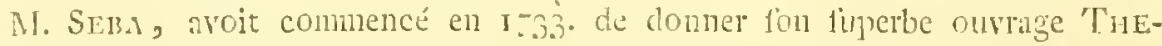
saurus rerum Naturalium en forme d'Atlis; le deux preniers Tomes parurent de fon vivant, mais venant a mouri" en 1 \%go. les desfeins et les planches du tome 3.4 . refterent fous les heritiers, qui n'etaient pas d'accords antre etax, enfin, ils parurent par les foins de M. M. Gavbits et VosmaER, le premier Tome contient les Quadrupedes, le fecond les Amphibies, le troilieme les animans de mer, Liobles, Oursfins, Poisfons, Comuilinges, et Zoophytes, le quatrieme les Papillons et autte Irlectes, les figures en font très bounes, dumoins la plupatt, mis entre les Infectes et Coquillages et ausli les ferpents, on voit fouvent trois ou quatre fois le mime, on en a des exemplaires eniminnez, mais ils ne pewvent plaire aux vrais Connoisleurs, premierement parce que les Serpents, Poisfons etc, y font peints daprès des objets en liqueur, Iti perdent toujours leur belles Couleurs, cn fecond lieu, les planches font trop noires daus les ombres, ce qui nuit benconp a une belle enluniuure.

M. Pallas, donna en 1760. fes Mifellanea Zoologiaa, avec des planches noires, trìs bonnes et il pourtuivit cet interesfant ouvage en I $67 \%$. lons le nom de Spicilegia Źoologica, done il y a paru jusqu'a prelent $1+$ Cahiers in Quarto, avec des figures tris exactes.

M. PENwant publia ell $\overline{-}_{6} \sigma_{3}$ fa Zuologia Britamia in Folio, arec des belles planclies enluminees; et donma ausil le mène ourrige in 3 ro. IV vol. avec des planches noires.

En 1:72. parnt 1a premiere partic de la Zoologia Danica fine Animalium Dania $\mathcal{E}$ Norregice rariorm ac minus notorm Icones, par M. Muluer, je n'en connais que les XI premiers Cahiers, arec des très belles figures, le même ouvrage parut en 1779 in 8 ro.

$\Lambda$ près ces Ouvtages qui traitent du regne Animal en General; je rapporterai ceux qui concetnent cliaque claste.

Entre les Ouvrages qui touchent les quadrujedes, Il y en a quatre qui meritent nutre attention, le preniet et le plus Celebre, elt 1'Hifoirs Naturelle Generale \& particuliere des Animaux, ayec ba defiription du Cabinet du Roi, que M. DE BUTTON, commencait a publier en $1 \% 79$, et qu'il a continné jusque a prefent; car apres avoir fini les Quadrupedes, qui Jui etaient comnues alors, et il lui viment tant des -quadrupedes nouvcax, et les nou- 
reiles decourertes ini ofirirent tant de matiere qu'il jugait a propos de donner des fiplplements. J'Hilioire des quadupedes, eft traitée en XT Volumes in 4to. et II, volumes de supplements $M$. d'Aubrenton, y joignit le itruelute interieure decrivant le fquelette, les parties de la respiration; nutrition et propagation de touts les Animiax que lui et M. de Mertred, araient disfequez, enfin on joignit a chaque Aninal la defeription de fes jarties qui font contervées dans le Cabjinet du Koi ; le même onvrage parur en mềne tems in 12vo. en 31 volumes et 4 volumes de Supplements, peu cle teuss après, il en parut une autre edition en treize volum. in I2vo. l'Ouvrige füt biettut reimprimé à Amfterdan in 4to. avec beancoup des additions de $\mathbf{M}$. le Proffesfeur Altanaxd; que M. Burfon a fait reimprimer dans fes supplements, on en a une traduction Al.LAMANDE par M. Martini de Berlin, une Hollandoile, d'après l'edition d'Amperdan, et on le traduit aculullement en Anglois. Cet ouvrage, a merité les Loungres de toute l'Europe fiavante, quoique il n’aye point echajpé a la critique, firr tout la Theorie, de la Terre le Sylteme des molecules organiques, la deneure de quelcues quadrupedes \&c. Les l'Janches furtout l'Edition Franciafe font tris belles, pourtant pas toutes d'une egale verité. On en a donné des enlmninéez mais je doute fi M. DE Burfon les a arouéz, jen posfede 150 . je ne feais pas, s'il y en font plus.

En 1:-1 - 15.5. les quadrupedes parturent en Coulenr a Tomis, fous le titre de Animale quadrupedi tolti dal Naturale disfegnati, inciff \& miniati col lor peri Colori da InNoc. Alessandrie Piedro Scatagida, Fol. 3 tom. quoique M. Erxlebien le dit en +. Je n'en vu que trois, les Animais font pour la plupart copiées de louvage de M. DE Burron, il y en a quelques Originaux", mais les deux demiere planches defigurent beaucoup l'Ouvrage; ils reprefentent lis Cirafje er le Buffle tirez de Joxstnon.

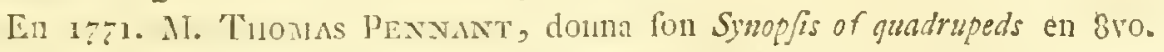
on les quadrupedes lont rangéz fuinnt le Sygeme de M. RAY, il refonda en 1762 cet Otrrage et y a ajouta un grand nombre des Animaux, et quelques nouvelles planches, mediocres, l'Ourrage parut en 2 tomes in 4 to.

En 1.:5. M. Scureber Profesteur en Hiítoire Naturelle a Erlangen publisic un Onvrage Altemaxd fur les quadrujedes intitulé Die Zumgthiere in Abbildungen nach der Notur, mit befchreibungen durch Jon. Chr. DAN Scrirener. Lirl. I.r. il y en a prefent, IV. Tolumes in 4 to. avec des Planches ienluniniltres exactes, crifes des Originaux et des meilteurs, Auteurs; ce Livre eft très efiimable.

11. Paltas, donna en Ir:g. fes Jpecies Animalium ex Glirim ordine, Erlang 4to. avec beaucoup des pianches trìs exacies et bien gravées, onvrage digne du nom de fon Celebre Auteur.

Ce font la les ourriges les plus eflimez avec des planches, qui traittent des guadrupedes; Les Oifenux ne manquerent dans le mème temps des excellents obfervateurs et desfinateurs, le premier qui donnait des figures asfez bonnes, fü M. F. Willulgany dans ton Omizirologia Libri III. Fol. Lond. 17z6; ils font rangís felon le synteme de M. Rax, qui a publie l'Ourrage.

En I 573 . partut Yurrage de M. Triscri en Allemand, fous le Titre VorRe?lung der V'oge! in Duitchland, Berlin, Fol. avec des très belles planches enluninées.

Lin 1738. MI. Eleazar Aibla donnait le Natural Hifory of birds a en III. $\therefore * 2$ 
Folumes in 4to. les Figures font bomes, mais Jes Enluninures tris mediocres.

Enfin le Celebre G. EDwards, commencait en 1--13. fon bel ouvrage fors le titre de Namural IJifory of Birds, qu'il continua jusque en $1: 5$ I ell IV. vol. in fro. arec des Planches très exacies et tris bien enlumincé, ce Livre meritant toutes Jes Eloges dant on le comblait, l'Autem fut encouragé de le pourlinirre fous le titre de Gleanings of Natural Hiflory exhibiting figures of qua-

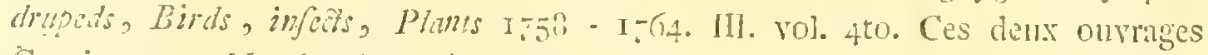
Contienuent $\mathrm{g}$ (1). planches très belles et exades.

Lin 1.31. M. CATESBY avait pubié fon Natura! Fifony of Carolina, Forida and the Bahama Jisunds cu IJ. Volumes Grand Folio, avee des figures très bien colorices des Quadrupedes, Oifeanx, Inleçes Amphibies, et Plantes.

Ces leux Ouvrages, dEDwhos et de CATEss. jartrent cu fuite enfemble duns la belle Collection de Neturnberg en 1.1. vol. in Fol. avec les bonnes Copies des figures originales

La France voyait en 1-60. paratre de Orvenge le julus etendu que on avait avant ceux de M. M. BuFros et LATtan; cetait M. Brisson. qui domait l'OrNmTHotogla five fynopis figens methodicas axism dirifiones, Paris VI. vot. 4 to. avec phus de 220 planches, d'olleaux, dork la plupart font tris vrayes.

Ces Planchescrecutées par la main du feavant $\Lambda$ rtifte MARTINET, nous montrent beancoup d'oifeaux qui n'étrient point decrits auparavant au nombre de Ilhs de 500, quoique ces Gravures ont étćz laites d'après Natme: on voit qlie pluiieurs font destinés d'après les oifeaux empaillez, et quelques un très mediocroment on n'y voit jas cette vie, ces attitudes vives, qui font le beau d'me planclue et que M. Enwards a fi bien filit.

En 1.65. il pant en Florence, J. Gerint, ormithologia edente LAurentro de LAurextus, VI. vol. in Folio, Je n'en ai vu qu wn Exemplaire rare avec des desfeins faits a la Gounche. Car il n'y a que neuf de cette forte, Jes oifenux ne font pas touts peints avec les Conleurs Naturelies.

En 1765. parut ansii le prenier caluer des j]anches en]uminées de M. d'AugENTON, fils du. Celebre M. d’AuBENTon, (qui avait dommé les Anatomies des quadrupedes dans l'Hittoile Naturelle de $M l$. BuгFoN, et fut continué jusque en I:ô et contient 1003 Planches; dome i] y en a 27 d'Injectes, 2 Anplibies, et 3 Coraux, touts les objets font fiats d'après Nature; et a desfeiu de fervir a l'Hiltoire Niturelle de M. liulfor, c'elt la Collecion a plus étendue que mous arons jusqu'a jrefent, des oileaux; et l'emporte ansí fur toutes les autres, par le nombre des Especes; toures les Planches font de la

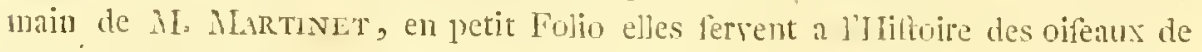
N. Bumfon, qui y renroye a claque oileau qu'il decrit, même on a une edition de lubitoirc Naturelle des oilcans, en petit Folio, faite expresfement pour ces planches. Ta premier destein des illuhires Autenrs était de n'en domner que 600 ou 800 , mais, la quantité des vifenux qui font au Cabinet du Roi, ceux que M. Burgon, trourait dams les Porteleuilses du Cheralier Brucr, et de feu MI Connerson, et autres, firent mouter le Nombre a toos planches, il y en a clonc neuf cens feptante fix planches d"oifeus, dont une grande pant contienuent trois an quatres Especes. Ces p]anches parurent en Caliers, fans ancun ordre fyfematiçue, et ainit pele mêle; et fans ancune deficription, feulement une etiquette arec le nom de l'oifeat, felon M. Brisson au bas de 
la Planche, pareecue M. DE Burron, refervait les Deferiptions pour fon Ourrage, qui parnt en $\mathbf{1}: \mathbf{c}^{\circ}$, jusque a prefent, ct femble lini a um Tome in 4to. pris, qui contiendra les Canards, Jetre's, Manchots, l'ingoins ete M. DE bufrox jugeait a propns, de changer les noms des obican dans fon Ouvrage, aree celui des Plinnches; et de les tirer ou des noms mationaux, ou felon leur Couleurs, er autres attributs. Plufieurs Amateurs avaient lait relier leur planches, felon les nombres comme il paraistaient; 11 . de Burrox, fuivic un toute autre arrangement dans fes defipiptions, ce qui me fit refolver de faire pour mon propre ufige une Table de ces planches, je la conftruifis, felon les noms de 21. DE Butron, et comme les Etiquettes des Planclies fe trourent fur chaqu'une d'elles, je ne jugeai pas necesfaire de les repeter, j'y joignis les noms de M. Brisson, quand ils font differents des Planches, et la planche de cet Auteur, quand l'oifeau y efi donné, ce que M. DE Burron, n'it gutre hait dans fon Hilkoire des Oifeaux, jy joignis en mème tems les

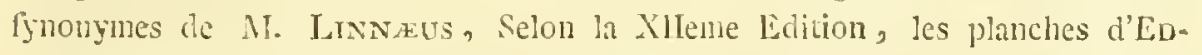
Wards, et la belle nomenclature de M. Lathan, aree les noms Anglois. Le ternier tome de $M$. DE Eutron, n'étant encor publié, j'ai fair ufage des noms de ceux de M. Brisson, quand l'Ourrage de M. Burron, partitra on pourra aifement y fupplier en marge; pour les lnfeces, je les ai notés felon les noms de 2I. Iannzeus et Fabricius fpecres infoclorum. J'ai laisfé en blanc les Infectes, que je ne trouvai pas chez ces grands Entomologues; en attendant les fynonymes que M. DE Nonvreillaro, qui travaille a prefent a certe branche, leur jugera a propos de domer; Javais fait cctte table pour mon plaifir et mon propre ufage, en y parlant arec M. LE Marquis de St. Simon, cet homme fi illuftre par fa naisfance que par fon erudition, me difait qu'il avait commencé une table, felon l'ctiquette des planches; les politesfes et les maryues d'eftimé que ce ficarant Mecene des fiences et des Arts, me arair toujours montré, me firent copier mon MSS, mais cependant je juggeni, que j'en pourrais faire plailir a quelques anis et feavants $\mathrm{cn}$ le faifant inprimer, jen'ai done fait tirer 50 Exemplaires; pour les difiribuer aux Amateurs; Il ne me refle a dire fur cette table, que j'al fait ufige de l'édition en I6 vol. in 12vo. de l'Hiftoire des oifenux; et qu'elle reparaitra peut être a la fin de mon Esfay orminhologique done je fuis occupé acituellement.

Un tel Ouvrage, que ces Planches enluminées; le plus grand que l'Europe litteraire a vu' jusqu'a prefent, lut recu, comme il meritait, avec beancoup d'empresiement; et d'approbation, les fcavants de Leipsicu neammoins en firent une Critique fevcre (voyez Conninentariz de rebus in fiemtia Naturali et Meticina gefis, vol. XXI. pag. 12. - 17. la plupart des fearants ornithologues fe tenaient au jugement d'Horace.

Hic moeret liber aera Sofis et trans mare currit, (voir p.xvi. pour la correction.)
Et longun noto, fcriptori porriget cenum, Sunt delicia tamen, quibus ignovise debenus.

En r 7 I. on vit paraitre a Rome, Cent-trente figures d'Oifeaux gravéz et Coloriez, en grand Folio, et on voyait avec fuprife, que dans ce fiecle des fciences et des Arts, on fcavait aller en arriere, au lieu d'avancer dans la carriere, les Oifeaux font tres unals desfinés en attitudes forcées et contre Nature, et 
cncor plus nual coloriés, daus le gout de ces Eannpes enluminés des faints, qu'on fait a Nurnberg, pour les Enfants et le bas peuple.

Les Ampinmes ne jouisfent pas du privilege d'čte repréfentés en Eftampes coloriés; nous n'arons tans cette branclie, que le bel Ouvrage de W. Röser Hilloria Naturalis Ranarum Nofratim en Latin et en Allemand, arec des bellès planches tris hien enluninces, chaque płanche eft double, donc l'ume ne inontre que les contours, 11. Tosmuer, a donné trois deferiptions aree des planches exactes et viayes du Serpent a fonnette, de deur Lezards Serpens, et de deus coleuvres a platte queve; et MI. VAx LIER, a decrit et douné, en Conleurs quatre ferpents qui fe trouvent dans le Païs de Drenhe.

Les premiel Ourrage des Poisfons, qui merite d'ère cité, eft celni de MrLuUGnry hiforia Piscium Oxf. 1686 Fol. avec des bonnes figntes noires.

M. KLEIN homme infatignble en Hitoire Niturelle, publiait fon Miforia Piscium. Misfus I - V. Gedani 1741 - I749. il y a quelques amphibies, les figures font pour la plupart très bonnes.

En I.49 on donna daus la grande Collection de Nurnberg les Poisfons et les Amplibies, que M. Catesßr, avait donné dans l'Ouvrage, que nous arions cité ci devant, cette Copie eft asfez bien faite, les deficriptio:s font en Alle. mand et en Latin, les Conleurs font pasfables, que l'on doit attribuer, en parlie au mauvais papier, qui fait fouvent beatcoup de tort aux Ouvrages Allemands d'ailleurs de leaticoup de merite; mon Exemplaire comfine en $\%$ o planclies, je ne feais, fil y cn part de plus.

En 1.57 M. Vosmare, doma l'Ouvrage de MT. Rexirn, Paisfons, Ecren yises 8 Crabes de divergos Couleurs (t Figures, que l'on trouve autour des Jiles Molucgues el fur les Cotes des Terres Aufrales, in Folio avec des planches enInmincez, je fuis occujé a donner une nouvelle edition de cet Ouvragie; en Francois et in Hollanduis arec des deferiptions et citations.

M. Schaefrea publiait en 3760 Cinq Pcisfons de la Baricre en planclies: euluninees, font bonnes arce une ronne defeription.

En т700-1770. Je donni quelques Poisfons et Amplibies, qui fe tronverent dans le Cabinet de leu M. Sculosser a Amperdam, in grand 4 to. avec des planches enluminées apris les fort bons desfeius, diun jenne pcintre a $\Lambda$ m. fterdan, que $\mathrm{M}$, Sculosser, avait dresfé dans ce genre de Peinture, ie les donnai en forme de Lettres, le premier liut le Chatodon Aigus, le fecond une rare Torme Cartilagineufe que M. PENNANT a donné après dans les 'Tranfictions Mhilofopliques de 17ax. Le troilicine une grande Grenouille blewe cu destus et Couleur docre cn desfous, la quatrieme un Chatodon il bandes et deux Epines a la machoire inleriente.

Enfiu ausii l'Hifoire Niturelle des Poisfons eut fon Buffon dans M. Buocus fiarant Medecin a liekun, qui donna un Guvrage Nlenand fur les Poisfois, dont it parnisfent 15 Calijers, avec des Planches enluminées, d'une vericé exacte et une defcription la quelle hat homneur a fon illutre Auteur; qui me mande, qu'on le traduit en Françis; ce fera une fuite au bel Ourrage de M. De Bufrox, qui ne le fera point tort.

Les Infegtes fi remarquables, par leur Mctanorplofe, leur maniere de vivre leur éclat chlonisfant, firent longtems le funct des l'eintres et giveurs. J'ni

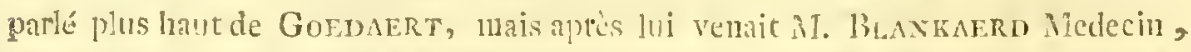
qui dounait un petit trates en ôro. Sous le Titre de Schoux:burg yan Runfen, 
zlingers, en vizgen, Amtterdam 1656. avec des bonnes figures en luminées i) y a quelques Infectes esotiques 111:is très pen, les desfins font fort bien faits et l'enluminure de quelques Exemplaires tris bonnes, ce livre eft fortrare a prélent.

A la fin du XVIr. fiecle Mlie MERtaN, partit pour Surinam, afin d'y obferrer les infectes de ces Contrées, elle avait de ja publie les Infectes de l'Alle. magne tous le Titre: Ruspen Vermandelung and Blumen nahrung. l'Metanor. phofe et nutrition des Chenilles, NornberG I6:9-16:5. 2 vol. in 4to. re. venue de Surinam elle donna fon grand Ourrage, fifoive des Infedes de Surinam, Amferd:un 1:-5. forme d'Aths. On joignit les planches de ceux de l'Furoye avec letr deferiptions dans cet Ouvage, en placant, trois ou quatre Planches fur une feuille, Mlle Mliruas, et fa fille aince firent quelques Copies en miniature et d'autres a dit gouache, et la fille enluminait quelques Exemplaires imprimés; on comprend aifement que ces desins et Exemplaires enluminés font très rares et fort payez font mers; J'enai vu d'autres enluminés d'après les Pajillons, qui font conferrez dans les Cabinets, de cette maniere jai enluminé mon exemplaire, et les Chenilles et plantes après un Exemplaire enluminé par la fille de M. Merrax, l'Ouvrage de Mlie Meruan, eft un des meilleurs, que nons arons dans ce genre.

En 1720. M. ALmis, domait fon Natural Hiffory of Englifeh infects, with 100 Copjeryłates Lond. 4to. Je ne le connais, que par le titre.

11. Eruscir, publiait de 1720-1728. fes defcription; des Injectes d'Allemagno cil Allanand, 4to. 13 Caliiers, cet Ouvrage eft trés inediocre.

Enfin parut l'Ouvrage de M, Reaunue, Memoires pour fervir a l'Hifoire des Infedes, Paris 17j+-1742. VI. vol. 4to. avec des lorts bonnes Planches noires; on en lit wue Copie a Amfterdam en 12 vol. Sro. avec des Planches, quelquelois au desfous du mediocre; la defiription eft de M. Reaunur, c'ett a dire an desfus de la louange er de la Crilique.

l'An. 1776. M. Rüse1, Peintre a Nornberg, donna fon premier Cahier des delices d'lnjeiles, en avec des tries belles plinches enlumines, il pourfuivit cet Ouvrage vrayenent fuperise, jusqu'a fir mort, qui arriva en 1761. il y en a 4 vol. dont le premier traité des l'apjillons de joul et de nuit, le feçond des Searabés, Grillons Sauterelles, le troifieme des Licreyisfes, Polypes, et le Quatrieme, contient quelques Papilions d'Europe et exotiques; font beafils KLEEMaN, en a domné une fuite de 40 Panches, cet Ouvrage elt traduit en Hollandois.

N. DE Geer Chambellan du feu Roi de Suede publiait en 1752 , le premier tome de les menoires pour Servir a l'lliloire des In/ectes, qui fut fuivi après quelques annces de V. Autres, et le Vll. Parut après la mort die l'Auteur, les defcriptions des Infecies font très exacles et belles, quoique point ecritcs avec cette beauté de fiyle, qüọn admire dans les memoires de M. DE REAUMur, les figures font fonvent très mediocres.

N. Albin donnait en Iбj6. fon Hiftoire des Araigneses en Anglais, in 4to. je ne l'ai point vue.

En 1757. M. CLERcs, domma me trìs belle defcription des Araignées an fuedois et en Latin avec des tris bonnes figures enluminces in $4^{\text {to. }}$

M. I. E. VoEt, melecin a la Haye, commencait en 1766. fon Hifoire Naturelle des Coleopteres en Hollandais, Français, et Latin, avec des très belles figures enluminées, d'ayrès les meillents Originaux, qui fe trouvent dans 
- ics Cabinets de la Frollanke; il y a jusqu’a prefent to Planches de la jremiere parlie, et j'en ai 40 du fecond. Les noms des Inferes y font arbitaires; fans citations ou fynonymes, et les deferiptions ne contiemnent, que les Couleurs.

M. Cramer, marcland a Anferdan, posiedant un tris beau Cabinet

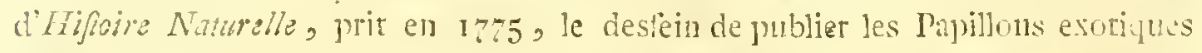
the fia Collection, et de toutes celles de la Hollanie, quili pouvait voir, il en publiait un très bel Ouvrage, qui parut en caliers au nombre de 36 , qui font + vol. in ftu. Les Papillons font très bien desínez, mais les Couleurs ne fon pas tunjours fidelles er les figures, quelques lois très roides.

Euviron ou peu avane M. Breny a I.ondres, donia un trìs bel Ouvrase , fous le titre de Illuflrations of Inferis Lond. 16. 4 to. 2 vol. aree des Manches fiperiemement enlunines.

En 1-бz M. M. Sepp. pere et fils, libraires a Amfterdan, nnntrerent ce que le burin et le Pincenu pencent produire, manies jar une uniin artiste, il commencerent, un Ourrage des l'apillons, fous le titre de Befichouming der wonderen Gods in de min/t geachts Shepzelen of Nederlandiche Infeiten, cet Ouvtage fe continue encore, on a to Cahiers in 4 to. Je n'ay jannais vu quelque chofe de pareil en eniuminure; ils font touts d'une verité et d'un Art ámant; on y troure la metanorphofe de cliaque infecie avec fon ocuf aggrandi et la plante dont il fe nourrit.

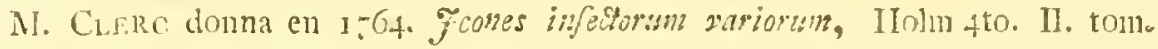
les Planches font d'une grrande beanté, c'eft dommage, qu'on a employé la cerufe au lieu du blane de coquille, de la tout ce qui devait itre blanc eft noiratre et l'argent qui brille fur quelques Plalenes fe noircit du noins duns l'exemplaire que j'ai ru.

11. Schneffer Miniftre Jutherien a Ratisbonne puthiait en torn le pre-

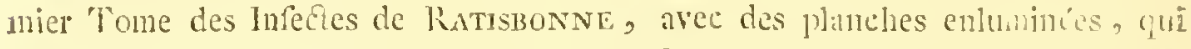
fut fuivi de deux autres in fto. les Infectes y font mis this ontre, en t.n voit que queiques un font enluminéz a la hâte, on n'y a aucune cicliription ou citition.

En I:30. M. C. Stoll, qui a continuć l'Ourrage des Papil'ons exoti jues après la mort de N. Cramer, publiait fon prenier Callier des Pumsijes et Cicades, dout il y a jaru de fa quatre livraifons, les figures font fijericurement enluminées, par la main Artiste de M. Sepp, et furpasfent beaucoup les Papillons exotiques, mais les deferiptions font confufes.

En 1782. M. PAllas, domia le premier cahier de fes Gones infeciorum Rusfice avec des planclies enluminées très bonnes, il fun̂it de nommer l'Auteur, pour juger favorablement de j'Ourrige.

Les, Cog̨uillages, qui font an jourdhui les delices des Naturaliftes n'onr point manqués des grarures; foit noires, foit enluninics.

En $1605-1692.11$. Lister, publia fon fynop/is methodica conchyliorzun in Folio, chaque coquille eft gravé, dans une jlanche ajart, et munerotéc, mais, puisque que beaucoup de ces planches, quil font an nombre de 1059. dans mon Exemplaite, furent égurées après la mort de l'Autenr cr même je crois de fon vivant, on n'eut point d'Exemplaire complet, mine celui de la Bibliotleque du Roi et de la Bibliotleque de Leide lunt defects (voyez le Catalogue de M. Davila et la Bibliotheque de M. Dr Bore, enlin M. Hutchisson trouwa la plupart des planches, de forte qu'il n'y unamuent, ques cinq 
ou fix, dans la nouvelle edition qui parut en 1rro. C'eft wil fort bon Onvrage, quant \&ux planches, mais tâns defcriptions.

11. Rumpir Conleiller a Ambone, avait decrit les Coquillages d'Aniborne, Boero, Uning. Cet Ouvrage fut plibilé par M. Schynvoet a Amferdum, qui y fupplea les plus belles pieces du Cabinet de M. D'A Quet de Delfe, eet ouvrage parut en $171 x$, quoique que quelques Exemplaires portent 1 :05, les figures font pour plupart trìs bonnes et exacies. Je n'en connas qu'un Exemplaire cnluniné, qui eft dans la posfesion de.ll. Vosmafre, latendant du Cabinet, du Prince Stadhouder, on en a une traduction Allemande par N1. Muldrar, avec des additions de M. CuEnsitz, le plus grand Conchylioiogue de notre fiecle.

En $\quad$ i +2 M. D’Argensville publiait fon Hifoire Naturelle echircie daus deux de fes Principales parsies, la Conchyliologia, et la Lithologie Paris, fto. Ein 1757. il donna la Conchyliologie a part, beaucoup atugnentée, avec des très belles Planches gravées aux depens de plufieurs Perfonnes de dititinciion; la Zoomorphote, que l'Autenr a ajouté a certe edition eft très infiructive a caufe des figures, des Animaux qui vivent dans les Coquilles.

En 17-8. parut une troilieme edition en trois vojunes in 4 to. avec beaucoup des nouvelles planches, mais trìs inferienre a ceux de la leconde; et les noms propres y font très fautils ct a peine reconnaistables.

Ein 1742. parut le bel Ourrage de M. Gualthiert, fous le titre de Nico-

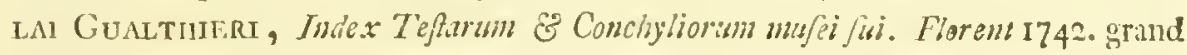
Folio, les figures font très bonnes et vrayes, et ont comme ceux de Lister l'avantage d'être gravés deux lois, deforte qu'on voit l'embonclure, des univalves et la charniere des bivalves; mais le texte efe feulement une defiription, des Couleurs des Coquilles, lin1s nonls, funs citations, outre qu'on y voit guelque fois laster et Rumpuns.

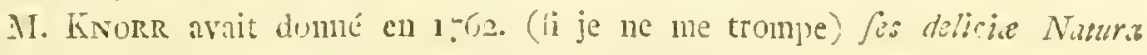
felecte en 11. vol. grand Folio, avec des Planches enlmminées ect Ouvilg ontient des Q Zuatrupedes asfez mediocres, quelque Poissons et Torines quelques Zooplaytes, et un nombre des Coybiiles, mais très peu des rares. Les figures font asfez bonnes et naturelles.

En 1744. il avait commencé un Ouvrage fur les Coquilles in qto. qui fe putbliait par caliers; nvec une courte defeription Allemande et Francaile. Nais étant decedé pendant le cours de 1'Ouvrage; il fut interrompu quelque tenus, mais fes heritiers aidez de M. Houttuis, medecin a Amferdam, (Celebre, par un tres ample commentaire fur le fytteme de la Nature de N. Lisweus, qu'il cont: nue encor ct dont il y a deja $3^{6} \mathrm{vol}$. en gr. $3 \mathrm{vo}$. avec des très bomes planches;) reprirent l'Ouvrage, gui confifte en VI parties, claqqu'une de go planches, excejte le tixieme, qui en a fo. M. Houtrun, fournishit les plus rares, que tun propre Cabinet et ceus des autres Amateurs lui offraient, et qui lont le $\mathrm{V}$ et VI Tome; les figures font asfez naturelles et bien enluminées, mais trìs peu font placés de maniere, qu'on voye la bouche ou l'Ouverture, ce qui lait douter des genres et les deficriptions, ne donnent que les noms que les Coçuilles unt ches les Amateurs, fans eıtitions d'auteurs; NI. SchröTer pasteur a Weinas, en a domé des fynonymes de linnzus, Murtisi, et Runpu, mais quelque fois tris fututives, voyez fon Jouraal pour les Armateurs

$$
x * \text { औ }
$$


de la Canchylioloris at de lin Lithologie, (Ouymage Nlemand tom. IV. et quelquics corrections tom. V.) M. Hovtruin, a donné cet ouvrage en Holl indris.

Entin pratut le plus bel Ourrage, que l'lurope a vu dans cette branche;

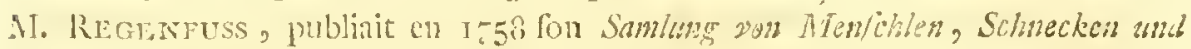
andern Schalticran (Colleciion des Coupulles bivalves univalves et autres), en grand Folio. Je n'en posiede que le a rolume, qui Contient ra l'yanehes.

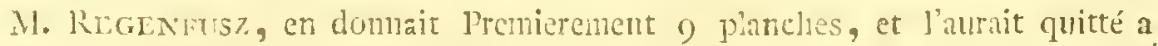
caufe des frais; mais le stind Necene, des Arts et de la Science Natureile le

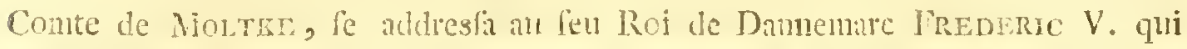
aprella M. Regtonusz, a Coppenhague, et prit toutes les depenles firr foun compte, un feavant Danois en fit la defeription, en Allemand te frangais, et il fut inprimé aree des tris helles vignettes en bleu et rouge; l'ourrage fut pret a paraitre, guand un ordre du Roi le fujprimait, et defendit la prblication; quoigue la deftription lit bonne, M. Craner vice chanchelue à Kiel et predicateur de la Cour, aidé de M. M. Ascanius, Spengerer, et fit tout de il. Lóratzinstein firent une nouvelie defcription benucoup meilleure, et lOuvrage parut, quoique les Exemplaires en font très rares, et tris chers.

I.ce fecond.'Tome, n'a jas paru, il n'y a que les VI. premieres planches flans les Cahinets de quelques Amateurs; la mort de Madame Recienorosz, que entuminait les figures, y methit prenierentent un abtracle, et on ćamait encor fumonté, quand un Courtifa pertuada le Roi Cirrasteen VIl. que les repenfes étaient trop gratudes; ct oque l'Ouvrage ne fe terminait pas avee le $\mathrm{T}$. Tome, ete. la desfus le Roi ordonnait qu'on fe faisirait de touts Jes Exemj)laires; qui apparemment ne reparaitront plus. —- Quand an Planclies, ils tont fuperieurenent executés imprimés en Couleurs et corrigés et finis parle Pincens.

Feu Martisi un des plus lenrants IIommes, que l'Allemagne a vu dans l'Ilifoire Naturel]e, publinit fon Cubinet des Coquiliages en Allemand, aree des Planches enlmuines très bonnes et vrayes, et uine Hiftoire Complette de chaque coquillage; l'Ouvrage parut in fto. en r 760 . et l'Auteur en donna de tems en tens des parties, jusqu'au tom. 11I. quand une mort fubite l'enleva au Sciences, mais ha fuite, par un bonleur très rare, fut mandée a denx Conchyl-

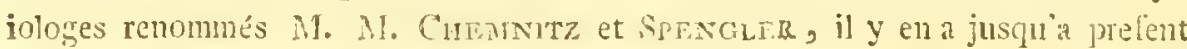
VI. Tomes c'eft l'Ourrage le plus complet, que nous avons dans ce sente, et les planches font tris belles, quoique beatcoup inferieures à ceux de 11 . Remenusz.

On trouve ausfi une asfez grande Cliantité des Coguillages, enluminés dans un Ourrage qui le pullié a HAlte, fous le Titte de Naururfors/cher, il y en a deja 20 vol. et dans les belles occupations des Naturaliftes asfociés de Berlin donc il y 8 rol. in 6 ro.

Sur les Ourfins de Mer nous n'avons, qu'un Onvrage compiet, dans la feconde edition de J. Tr. KuEn, Naturalis dispofutio Echinodermatum que M. LFSKE, m des plus feavants Naturalifies de l'Allemagne à amsmenté de 93 Especes; Ourrage magnifique jublié en rars, a Teipzich in 4to. plejn des bounes figures, des bounes deforiptions, et des idées neuves et juftes.

Les Zooplytes, donc l'Hiftoire eft encor au berceau, n'ont que trib peu des figures, et feulement un Uurrage enluminć, M. l'ALtas, l'Aritote du Nord, 
arait donné l'esperarce, qu’il cn domerait un Ouvrage enluminé in Folio, mais fes voyages et fes autres occupations fcavantes, l'ont empeché jusque prelènt.

Les Figures que leu M. Donatr a donné dans fon lifforia Naturale marina dill Adriatico faggio Vonez 1750. 4. font très pell, et pell correctes.

En 1755. M. Exurs marchand a Londres, obferva les Zooplyytes au bords de lin mer de Cormall et autres rivages de l'Angleterre, il était accompagné du Celebre LuR r.t un des plus grands Peintres de l'Angleterre, de ces feavantes recherches et destins, naquit l'Ouvrage intitulé An Effay towards a Naturab Hillory of the Corallines, Lond. I 555 . 4to. avec 38 planches très belles; on en a quelques Exemplaires enluminés; c'eft Ouvrage elt traduit en Français,

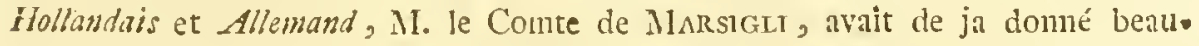
coup des figures de ces Animaux plantes, dans fon 1 ifoire de la Mer Adria. tique 1725. Fol. avec beancoup des bonnes figures et quelqu'unes Mediocres.

Feu M. Baster favant Medecin a Ziriczée en Zeelande un des premiers Heros pour l'Animalité des Zoophytes, publinit fes opuscula fibcefiza in 4 to. II. parties Harlesm 1759-65. on y trouve beaucoup des Sertulaires et autres, que ce fcavant avait oblèrvé fur les cotes de la Zeelande, les figures font rutdes; on en a quelques Exemplaires enluminús, je poslede les deshins Originaux qui ne font pas finies de main de Maitre.

M. Catteshy en a dommés quelques uns enluninés dans fon bel Ouvrage que j'ai cité plus haut, M. KNorR, donnait benucoup des Gorgonia et des Madrepores dans les delicice natur.e.

Ce font la les Ouvrages les plus interesfants de l'Hiftoire Naturelle avec des planches, que je ai vu, ou que je posfede; je n’ai jugé que des Planches; lans toncher aux Ouvrages même done je me referve a parler dans mon introduction l'étude de l'Hiltoire Natureile; et je n'ai parlé que des Ourrages que j'aì même vu, je me fotunets cependant volontiers au jugement des feavants en ce genre, et je ferai content, fi cette courte notice pourra être utile, a cenx, qui commencent l'útude de l'Hifoire Naturelle; et qui voudraient former une bibli. otheque interresfante.

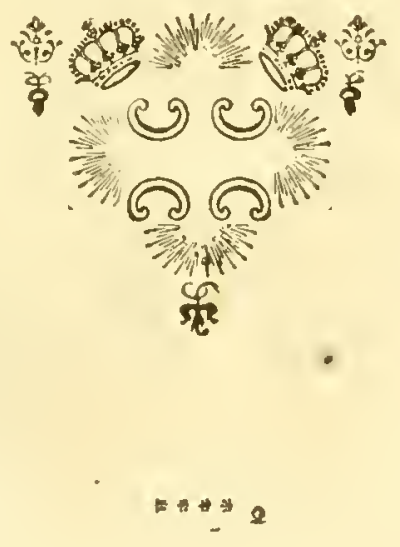




\section{CORRECTIONS.}

lag. 17. Jigne. des fous Menibre ete.

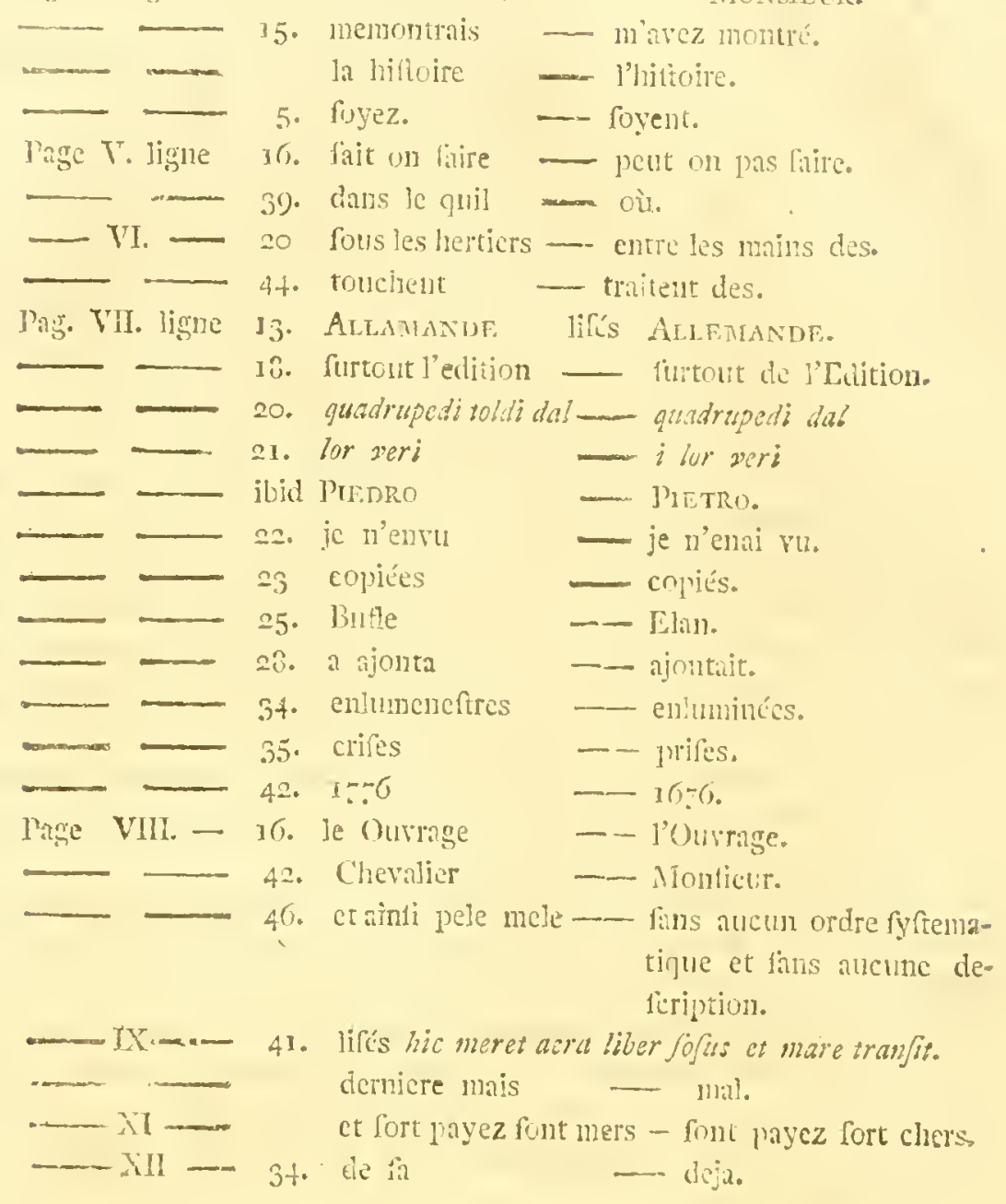



P1. 1. Tage Cocq. Phafianus Gallus. Limn. Ger. 101. I. Brisson Ornith. I.

2. Merle. Butron Ois. VI. I. Briss. Omith. II. p. 227. 11. 10. Turdus Merula Lins. Germ. I07. n. 22.

s.fig. I. Charbomiere BufF. X. p. 99. Briss. Ornith. III. p. 539. 1. Parus Major Linn. Gen. I16. 3.

2. Mefange blene Burf. Ois. X. p. r27. Briss. Ornith. III. p. 544 . 4. '"arus Coemuleus Linv. Gen, 116. 5.

3. Nomette cendrée, Bufr. X. p. 1 15 . Mcfange des Marais Briss. Orneth. IlI. 555. 7. Parus Paluftris Lins. Gen. I 6.8.

4. 1. Charoonneret. Bufr. VII. p. ع6I. Briss. Ornith. III. p. 53. I. Fringilla Cardutis Limn. I 12.7 .

^. Charionneret blanc. Briss. Omith. III. p. 60.pl. IV. fig. 4. varicté dis Chardonneret.

5. I. Petit cul jaune de Bengalc, Buff. V. p. 340. Carouge de Mexique Briss. Ornith. II. p. 118.23. pl. XI. lig. 2. Oriolus Mexicanus I.Inv. Gen. 52. I3. Edw. glean. pl. 243. Leffer Bonana Bird. LATHAM Birds I. p. $33^{8} .25$.

2. La femclle Carouge de St. Domingze Briss. Ornith.II. no. 25. pl. XII. fig. 3. Oivolus dominicenfis Linv. Gen. 5=14. St. Domingo oriole L.iThian lBirds I. p. 439. 26.

6. I. Moincau Buff. VI. p. 218 . Briss. Ornilh. III. p. 72. Fringillı Domefitica Linv. Gen. 112.56.

2. J.c Foudi (Friqnet) Burf. VI. p. 238. Fringilla Carduelis Capenfus Linn. 112. o. Briss. Ornitt. III. o.

7. 1. Taugara Septicolor. Burr. VII. p. 393 . 'Tangam Bruss. Ornith. p. 5. pl. 1. fig. I. Tangara Tatus. Lnv. Gen. III. 11. Pams Paradipucus Low. III. Tab. 349 .

2. La Houpette (Tangara) Berf. VII. p. 336. Tangara hupé de Caycme Buss. Ornitio. Suppl. p. 65. pl. IV. fig. 3. Tumagra Crifiata - Linv. III. $=4$.

3. 1. Veuve a quatre brins. Buff. VII. 226. Vidua viparia Veuve de la Core d Afique, Briss. Omith. III. p. i 39. Emberiza regia Linn. Ge11. I I $0.2 \% 3$.

.. Veurc Domicaine Burf. VII. 22S, la grande verve Briss. Omith. III. 27. pl. VIHI. fig. 4. Emberiza Vidua Lins. 1 10. 21.

9. I. Langrayen Piegriefche Burr. It. p. 89. Lamius Leacorynchos Lisx. Mantis 524. Piegriefche des Manilles Briss. Ornith. II. p. I 80. pl. I 8. fig. 2. Lathan Syn. I. p. 181. m. 33 white billed Thrike.

9. 2. Picgricfche rousfe Buff. II. 66. Piegricfehe du Cap. de bonne Esperance Bross. Ornith. II. p. 182. 18. pl. V. 1. Lanizts Collaris Lins. Gen. 44. 12. wood Chat thricke Lath. Birds.I. p. 169.

10. Cunnepetiere Femclle Buff. III. p. 57. Outarde Briss. Ornith. V.p. 24. pl. II. f. 2. Otis tetrux LinN. Gell. 953. Klcine trapp.

I I. Founingo (Pigeon.) Buff. IV..... Pigeon de Madagascar Briss. Ornith. I. p. I fo. Tab. XIV. I. Columba Mudagas;arienfis, Linn. Gen. I04. 21. 
11.

Ia. Ara rouge (Perroquet) Burf. XI. p. 250. Am du Brefil Briss. Orititi. IT. p. I 84. pl. XIX. I. Pfittacus Matao Linv. Gen. 45I. Red and bluchitcaw. Eow. pl. 153. Lathan bird's I. p. I99. I.

3. Amazone janne (Perroquet) BupF. XI. p. 30 \&. pl. X. Perroquet Juine Briss. Ornith. IV. p. 306 . Pfittacus luteus Lins. o. Aurora Purror LATH. birds. I. p. joI. 1. zo6.

14. Petit cacatoe a huppe jaunc Burr. XI. b. 1 29. Cacatun. Ie Cacathocs Briss. Oimith. IV. p. 204. pl. XXI. P'ittailis Criftatus Linv. 45. 22. Idfier withe Cockatoo Edw. Glcan. pl. 317. Latro. bircis. I. p. 258. 13. (14.

15. 1. Scarabaens Aloeus Linvo

2. • . . : .

3. Scarabaeus Bilobus LinN.

4. . . . . .

5. Scarabizeus Actaeon. Livn.

16. I. Scarabaens Fillio. LinN.

2 Scarabaeus Metolontha. (le Ilmecon) INw.

3. • • • . • •

4. . . . . . .

57. 1. Papilio Patroclus. Linn.

2.

3.

4. S. Papilio Cupido LanN.

7. . Sphinx Hyaiduthus Cravi. Tab. 36. F.

8. $\{$ Sphinx Bicolora LanN.

1 1. . Papilio Aetholus Sulzer Kenzcich. Tab. 19. Ij. I0. 11.

19. Papilio Hecuba Lins. mantisf.

à. 1. Papilio Idea Linn. la veuve.

2. . . . .

今. . . . . .

21. Crapaud dorfipare Rana Pipa Linn. Gen. Iøo. I.

22. Le Chamalcon. Chamdeoin (Lacera) Linn, inュ. no.

23. Manchetre de Nepume, Millepora Retepora, Paldas Elench Zaoph 148. Millepora Celluloja Linn. Gen. 333 5. 49.

24. Corail rouge. Ifis Nobilis. Linn. Gen. 340.6. P.LL. Elench. Zooph. p. 142.

25. Cannepetiere mâlc Burf. III. p. 57. Briss. Ormith. V. p. 2\%. Otis tetrax. Linn. Gen. 95. 3. voyez li femelle pl. Io.

36. Loriot Burf. V. p. 349. Briss. Ornith. II. p. 320. Orialus Galluilu, Linn. 52. I. golden oriole Lathan Syn. I. p. 449. n. 4.3*

27. Perdrix Bufron IV. p. I62. Briss. Ornith. I. p. 219. Tetrus Perdis. Linn. Gen. I I 3 . I 3 .

23. I. Bergeronnette jaune BuFf. IX. p. 388 . BRISs, III. p. 47 I. pl. XXIII. 3. Motacilla Flavib. Linn. I 4 4. 12. $\beta$. 
2. Bergeromnette du Cap de bome Esperarce Burfon IX. p. 396. Briss. HI. p. $47^{6}$.

29. Hibou ou moyen Duc. Bufr. 11. p. 103. Erass. Omith. I. p. 486 Stria Otus Linn. Gen. 43. 2. Long Eared owl. LarHan. birds. I. p. III. ก. 5 .

3o. I. Bruant Bitrf. VIll. 51. Briss. Omití, III. p. 258 . Emteriza. Citrimella Lins. 110.5.

a. Bruant fou Burf. VIIl. 68. Butmentes Prez. Briss, Ornith. IIT. p. 266. Enberiz a Ga Lanv. Gen. 110.11.

3. 1. Piegriefcte rousfe (La fenelle) Vid. pl. 9. 2.

2. Ecorcheur (Varicté) Iivur. II. Piegricfche rousfe Briss. Ormith. II. p. 152. Limius Colliwio (B) Linn. +1. 12. Red Backed Shrike Latri. Birds I. p. 16?. 11. 15 .

32. 1. Vuricté đlu mêtne, vid. plo 445 .

2. Le Tcha-Rert Burr. II. p. 95. Lanins Violacens Linn. Gen. 44. o. Petite Piegriefche verte de Madagafear Briss. Ornith. 11. p. 195. pl. XV. 3. Green Shrike, Lath. Birds. 1. p. 170. 30.

33. I. Tingara Tricolor. Bufr. VII p. 320. Tanyana Cayanenfis Cyanocephalos Briss. Ornith. Suppl, p. 62. pl. IV. t. 2. et p. 99: ple IV. f. I. LINN. O:

2. Tangana à tete bleue Bufr, ibid Briss. ibit.

34. I. Manakin in tete dor Bufr. VIIr. p. i61. Briss. Omilh. IV. p.448. pl. 34. 2. Pifral Erytrocapilla Linn. Gem. II. 56.

3\%" 2. Manakin a tere blanche Burf. VIII. p. i61. Briss. Oimth. pl. XXXIV. 2. Pifra Lencoseplala, Livi. Gen. $115 ; 8$.

3. Manakin rouge Butr. ibid. Manahin is tete pure Briss. Ornith.IV, p. 450. l'ifra Erythrocephalu Lixv. in G. CND, Le trois Es.

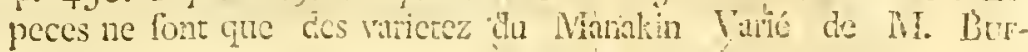
FON.

35. Mascarin. (Perroquet) Bufr. XI. p. I68. Tab. V. Brtsis. Ornet/. IV. P.

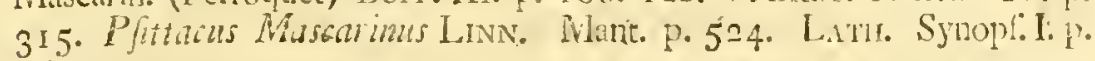
265.13 .72 .

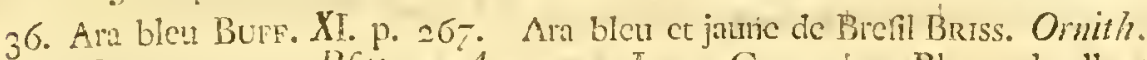
1V. p. 193. 20. Pfitacus Airarmenc. Linn. Gen. $45 \cdot 3$. Bluc and yellow Mlacaw. Latn. Synopl. I. p. 204. n. 4.

37. Cardinal Huppé. Buff. VI. p. I85. pl. 9. Gros bec de Virginie Briss Ornith. p. 253. Loxia Cardinalis Lins. Ger. rog. 3 .

38. Le Saracou Bufr. XIV. 38. Cochlearius fuscus Briss. V. p. 509? Can rroina Cancrophaga, Lins. Gen. 83.2.

3*). Cocq de Roche Buff. VIII. p. 180 . Rupicola Briss. 1V. p. 23\%. pl. 3 f. 1. l'ipra rupicola Linv. Gen. 115.1.

40. 1. Scarabaeus Claviger, Linv. mantif. 529.

3. Scarabaeits Tithyus, Livn. p. 542.5.

3. Scarabaeur Gideon, Linv.p 54 r. 2.

4. Scarabueius

5. Scarabaeus Diflzotomus, Linv. mant. 529.

41, I. Sarabaeus longimamus, Linn. p. 542. 39. 
P1.

fig.2. Srarabaents Hercules, Linn. 54I. 1.

42. I. Phalena Hesperus B. Linn.

2. Phalitina Cytherea, Fabric.

43. 1-g. Papilio Pammon, Linn.

2. Papilio Phorbanta, Lins.

44. I. 2. Papilio Protefilaus, Linn.

3. 4. Papilio Demoleus, Linn.

45. - Papilio Priamus, Linn.

46. - Lezard volant. Draco rolans. Lann. I. 21, I.

47. Nadrcpora Aiba.

48. Gorgonin Miniacea. Past. Elench.

49. Phaifan Bufr. III. Briss. Ornith. . . . Mhafiamus Gallus Cirjt.tuls. LinN. IOI. I. $\beta$.

50. Casfenoix Burf. V. p. i68. BRiss. Omith. II. p. 59. Tab. V. I. Corsus Caryocataites. Linn. 50. 10. Nutcraclicr EDw. $240.1 \mathrm{~A}$ trian Birds I. p. 404. n. 38.

51. Varicté de pl. 406.

52. Hupe Burf. XII. p. i 16. Briss. Ornith. II. p. 455. Upupa epops. LinN. 64. I.

53. Ore Tadome Rurf. .... Briss. 17. p. 344. Tab. 33. 2. Anas Tadorna. Gen. 6\%. 4 .

54. I. Pinfon Bufr. VIII. 155. pl. 4. Fringilla Briss. III. p. 1 f8. Frimgilla Coelebs, Lann. I I 2 . 3 :

2. Pinfon d'Ardennes Buff. VII. p. 175. Monti Fringilla, Briss. Orzith. III. p. I 55. Fringilla Montifringilla Lunv. I 12.4 .

55. Moineau Buff. 17. p. so8. Briss. Ornith. III. p. 72. Fringilla Domeltica Linn. Gen. I I $2 \cdot 3^{6 .}$

=2. Paroare Burf. VI. p. $\approx 44$. Cardinal Dominiquain, Briss. Ormith. III. p. I I6. pl. VI. f. 4. Fringilla Larvata, Lins. o.

56. Gonolck. (Piegriefche) Buff. II. p. 96. Lanizs Barharus Briss. Omith. 11. p. I 85. Tab. 17. f. 2. Linn. Gen. 44. I S. Barbary Shrilie, LAt11ant Birds. I. p. 173.23 .

57. Guepier verd a queue d'azur Betr. XIII. p. 206. Grand Guepicr des Philippines Briss. IV. p. 56 60. Merops. Philippinus, Linn. Gen. 65.5.

58: I. Lavandierc (varieté de Ia.)

2. Figuier tacheté Bufr. IX. p. 414. Ficedula Canadenfis, Briss. Iits. p. 492. pl. 263. Motacilla Camadenfis, Linn. Gem. I 14.0.

3. Figuier Orangé Bufr. IX.p. 46 r. Briss. Ornith. III. o. Motacilla aurantia Lins. 0.

59. Peterel du Nord. Burf. .... Petrel Cendré Briss. Omili. I7. p. 143. Tab. I 2. f. 2. Procellaria Glacialis, Linv. 70. 3.

60. Moincau de Guinéc. Buff. XI. p. 230. Pfittacula Guieufis, Briss. JV. p. 387 . Pfittacus Pullarius, LinN. 45 - 45. Red. haaded. Parakeet. Ëvw. Birds 237. Latiani Birds I. p. 309.

6ı. Perruche a tete bleue Buff. XI.p. 208. Pfittaca Amboinenfis varia. Briss. Omith. IV. p. 364. Linn. 45. o. Mantiff. p. 524. Pfittacus hamatodus. Red. breafted. Parrot. Latham Birds. p. 212. 14. EDW: 202. 
62. Nîrtin pecheur Pie Burf. XIII. p. 273. Ispida exalbo nigroque varia Briss. Ornith. IV. p. 520. pl. 39. 2. Alcedo rudis, Linn. Gen. 62. 12.

63. I.c Flammant. Buff. XVI. p. 292. Briss. Ornith. VI. p. 532. Tab, 47. 1. Phanicopterus ruter, Liñ. Gen. 79. I.

64. I. Comambyx Longimanus, Lixv. 204. I.

2. Cormbyx. • . .

65. 1. l'Arumatia. Mantis Gigas; Linn. 220. 3.

2. Lal fraife de St. Domingo, Mantis Podagrica, Lins. 220. 4.

66. 1. Phalana Hesperus, Linn. Gen. 233 . I.

2. Phaluzua Tau, Lins. Gen. 233 .

6\% 1. Arlequine Phalena Militaris, Lins. Gen. 233.

2. 3. Bergame de Martinique, Phalena Geometra.

3. 4. La Confole Phalenal Geonetra.

68. 1. 2. Alpicola. Papilio Apollo, Linn. Gen. 232.

3. 4. Le Velouté. Papilio Antiochus; Linv. Gen. 232.

69. Le Feftomé Papilio Thoas, Linn. mantus.

7e. I. Le Rocou de St. Damingo. Papilio Fucatus, mihi.

2. 3. Lc Nacré de St. Dómingo Papilio Heliconius Vamille, Liñn, $=32$.

4. La Quintcraye. Papilio Heliconius Thallo, Linv. 232.

71. T. Page de Cayenne. Papilio Leilus, Linn. Gen. 232.

2. 3. Papilio Chiron. Fabricin n. 40.

4. 5. Papilio Ruralis. Eryx. Fabric. $33^{6}$.

72. I. 4. Le Parafol. Papilio Doris, Livx. Mantisf. $53^{6}$.

ऽ. Le Tufctas rajé.

3. 4. Le Guidon: Thales Craner Exotic. Tab. 38. C. D.

-3. Le grand Cocq de Bruycre BurF. 111. p. 27t. pl. 15. Urogallus major Briss. Ormith. 1. p. i8ะ. Tetrao Urogallus, LinN. I03. I.

74. La Femelle.

75. Etounnean BífF. V. p. 241. Briss. Ornith. II. p.' 439. Sturnus Villgaris, Linn. 106. i:

76. Corncille Nanteleé. Burf. V. p. 83 . Briss. Ornith. 11. 19. Corvus Comin, Lins. 50. 5. Hooded Crow. LATH. I. p. 3I4. 5.

7\%. Alcyon (Alartin pecheur) BufF. XIII. p. 242. pl. 9. Ispida BRIsson Ornith. 1V. p. 474. Alcedo Ispida, Linn. 62. 3.

73. Le Pauxi Burron IV. p. I42. le Pierre Briss. Ornith. I. p. 3०3. Grax Pauxi, Linn. Gen. 100. 5 .

79. Loriot de le Chine Buff. V. p. 361. Lorior de Bengale Briss, Oimith. 11I. p. 329. Oriolus Melanocephalus, Linv. Gen. 52. 3. gुolden Oriole LATHAM birds. I. p. 45 I. n. 43 . A.

30. Corlieu Burf. XV. p. 53. Corlieu rouge du Brefil Briss. Ornith.

V. P. 544. Tantalus ruber, LuNN. 85. 5.

81. Le même adulte.

S. Le Toco Buff. XIII. p. 176. Briss. Ornith. o. Linv, o. ad Ramphaftos, Latiana bird' I, p. 325. pl, IX. n. I. 
83. I. Le grand foumanga. BufF. XIII. Grimpereau a longlic quetue cit Cap. de bonne Esperance. Briss. Ornill. II. p. 647. pl. XXXIV. I. Certhia famofa, Linn. Geib. 65. 20: Famous Crcoper Latir. birds I. p. 720 .

2. Guitguit noir et bleu Bufr. XIII. p. 29r. Grimpereau bleu dit Brefil Briss. IIl. p. 6ะ8. pl. XXXI. 5. Certhia iyanea Linv. Gon: 65. Sp. 94. black and blue Creeper. Latr. birds 724 .

84. Femelle du Perroquet Lori a Collier Buff. X1.p. 180. Lori de Ceram Briss. IV. p. 215. Pfittainus Garmlus. Linv. 44. 21. Ceran Lory, Lath. birds I. p. 268. n. 75. voyez le male pi. 119. Purpie Cippes LORY, Lath. I. p. 271.77 .

85. Perriche Emeraude Burf: XI. . . Brisso o Linn. Gen. 44. C. Bmeania? Parrot. Latil. birds I. p. 230.17 .32$.

86. Hocco Burr. IV. p. 128 . tab. 4. Briss. Ornith. I. p. 305. Crin Aile:tor Linv. Gen. I00. I.

87. Le Pelican Butr. XV. p. I. pl. I. Bk1ss. TI. p. $5^{19}$. Pelicatus Onociotalus, Linn. 7?. I.

88. Rollier d'Angoln BufF. V. 197. Gilgulus Angolenfis, Briss. II. P. T2.

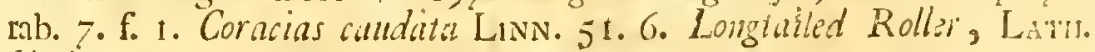
birds I. p. 409. 4.

89. Breve Burf. VI. p. I 2 1. Turdus brevicauda mili, Linn. o. M. 32.2. Lath. birds I. p. 393. n. 3\%. A. Merle a Tete noire des Mijlucces Brtss. Ornith. II. p. $3^{19}$.

90. 1. Cerambyx Hottentota, FabRic. 221.22.

2. Lucanus?

3. Le Buflard de Cayenne, Cerambyx Cerviccinnis, Linv. pl: 22. 3.

91. 1. 2. Le foleil levant, Papilio Callirrhoe, Fabnen 100.

3. 4. l'Esclavage. Papilio Lencothoe, LinN.

92. 1. 2. Le foleil couchant de Surinam: Papilio Euippe, Fabricir 19:。

3. Le point du Jour de Cayenne Papilio Carica, Fabr. $5^{62}$.

4. Le Crepuscule de Cayenne. Papilis Phardrus, Fabno 566.

5. 6. Le Chevron brifé, Papilio Ocirrhoe, Fabr. 329.

- 8. Papier marbré, Papilio Amphinome, FABr. 427.

93. 1. 2. Leopard de In Chine. Papilio Nifhe, FABr. 463.

3. 4. Le parqueté de Surinam. Papelio Hyparethe, Liss.

94. I. 2. Le Trapu male. Papilio Afterie, Linn. ${ }^{3} 32$.

5. 6. Le Royal Papilio Amatkea, Linn. I74.

95. 1. 2. Le Verd deau. Papilio Dido, Linn. 19=.

3. 4. Le Souchet.

96. I. 2. Grand Paon bleu de Cayenne. Papilio Idomenetes, Lrix.

97. Dindon. Buffon III. p. 187. pl. 3. Briss. Ornith. I. p. 1 j8. pl.XVI. Meleagris Gallo paivo, LinN. 29. I.

98. Poule de Japon. Varieté du Cocq. pl. I.

99. Grosbéc Bufr. VI. p. 165. pl. 8. Briss. Omith. III. 219. Laxiat Cocothrauftes, Linn. 109. 2. EDw. birds 188.

ioo. La Femelle.

ior. J. Varieté du Grosbéc de Coromandel. 
1.

2. Le Quadricolor Buff: VI. p. 199. Grosbéc de Jàva. Biriss. Ormiti. 1Il. p. 237. Loxia malaca, Lins. p. 109. I 6́. EDw. Glean. tab. $355^{\circ}$

102. I. Ani des Paletiviersi Burf. XII. p. 93. Crotopharas major; Briss. Oruith. p. i 80. pl. XVIII. 2. Crotophaga Ani, Lins. 4I. 4. Greater Ani, Latham Birds 16̈. 2.

2. Ali des Savanes. Buff. XII. p. 89. Crotophagus Briss. IV. p. 177. pl. XVIII. I. Crotophaga Ambulatoria; Ians. 41. 2. Lesfer Ani; Latis. birds 1. p. 360 . I. pl. 12.

103. Cartinal dominicain huppé. Bufr. Briss. Ormith. o. Frivgilla Domio nicana Crtftata, milii LiNN. 0.

104. Grofle Gelinotte de Canada. Butr. III. p. 405. Briss. Ornith. p. 212. Tetrao Cupido, Linn. io 3.5 .

105. Gelinotee des Pyrenées. Burr. III. p. 352. tab. 8. Briss. Ormith. I. p. I95. pl. XIX. Alchata. Linv. 103. 1 I.

106. La Femelle:

107. Anhinga roux. Buff. XVI. 26I. Briss. Oinith. VI. p. 477. Plotus Anlinga, Linv. 73. 1.

1o?. La Peintade Burf. III. p. 232. pl. 4. Briss. Ormith. I. p. 476. tab. VIII. Numida Meleagris, Linv. 102. I.

109. I. Majan Bufr. VII. p. 153. Maja Jmenfis, Briss. Ornith. III. p. 2 I 2. Fringilla Maja, (B) Linn. Gen. i i 2. 35.

こ. Maja Burf. VII. p. 150. Maja de l'Ifle Cuba, Briss. Ornith. III. p. 1 1 2. pl. VII. உ? Fringilla Maja, Linv. Gein. 1 I 2. 35.

3. Le Grenadin. Burf. VII. 23\%. BRiss. Ornith. III. p. 216. pl. IX. 3? Fringilla Granatina, Live. 112. 1 I. EDw. Birds. 191.

i io. Pigeon Romain Tacheté. Burf. IV. p. 330. eft une varieté du Pigeon Mondail.

11 I. Founingo bict. Buff. IV. p. i I . Pigeon ramicr blen Briss: Ornitho I. pl. XIV. I. LiNN. o.

1 1 2. I'Orfinye (Aigle) Buff. I. p. $15 \%$ grand Aigle de Mer; Briss. Omith. I. p. 437. Falco Osfifraga, Linv. Gen. 42. 4. Sea Eagle Lath. birds I. p. 30 :

11.3. Blanche raye (Etoumeau) Burr. V. p. 269 . Briss. Ornith. o. Sturims alli florur, mihi Linv. 0 .

114. I. Le Taita Buff. VII. p. 419. Tangara Brafilienfis luteo nigra, Briss. Ornith. III. p. 31. pl. II. f. 2. Tangara violacea, Livin. I I I. 5.

2. Varicté du Taita.

3. 'Tangara Negre. Buff. VII. p. 419. Tangara Cayanenfis nigra, Briss. Ornith. III. p. 29. pl, II. fig. I. Linn. III. o.

I1 5 1. Bengali Bufr. VII. p. 1 3\%. Bevigalus Briss. Ornith. III. p. 203 . pl. X. I. Fringilla Angolenfis; Linv. 112. 31. Eow. birds i 3 I.

2. Bcngali brun Burf. VII. p. I 35. Bengalus fuscus, Briss. Ornith. III. p. 205. Fringilla Fusca, Linv. i 12. 32.

3. Bengali piqueté. Burf. VII. p. 138. Bengalus primctulatris, BRiss. Omith. III. p. 200 . pl. X. 4. Fringilla Amandara, Linn. Gen. 112 . 10. Amandabat EDw. glean. 355. I.

I 6. Turvert. Varicté de Ia Tourterelle veite, Buff. IV. p. 39\%。

$\mathrm{B} 2$ 
13.

11 . Pic noir a döninó rouge. Burs. XIII. 83. Picus virginianus Eythic. cephalus, Briss. Ornith. IV. p. 52. pl. III. 1. Picus Erythrocephalus, Livn. Gen. 59. 7. Red headed wood pecker, LAth. lirds I: p. 361. Catesb. I. pl. 20.

118. Pigeon de Eanda. Buff. IV. p. 379. Faifan Cóuronné des Indes, Briss. Ornith. I. p. 279 . pl. VI. f. 1. Columba corcnata, Linn. Gein. 104. 17 .

ing. Lori a Collicr Burr. XI. 180. Loritus Ceramenfis, Bkiss. Ornit/2. p: 222. pl. XXIV. 1. PJittacus Domicella, LiNN. 45. 25. bluc Capped Lory, Lath. Birds 1. p. 27 1. 77.

120. Perroquet Tapiré. Buff. XI. 332. Tab. I I. Pfittacus Guttatus o. ND. rexifte pas dins la Nature, les taches rouges fe font par art:

121. Faifan Bufr. IV. p. 60. Briss. Ornith. II. p. 26:. Phafanuis Colchishis, LinN. 101. 3 .

122. Ia Femellc.

123. Faifan noir et blane de la Chine. Buff. IV. p. 107. Briss. I. p. $=76$. Phafiamus Nycthemerus, Linv. io1. 6. Edw. Birds 66.

124. La Femelle.

125. Hocco du Perou Lurf. IV. i 30. Briss. Ormith. I. p. 305. Crux Alector, Linn. IOI. I. Yoyez pl. 86.

126.2Lagopede fraife Burr. IV. p. 28a. Caille des Philippines Briss. Orihlit. I. p. 254. pl. XXVI. Tetrao Chinenfis, Linv. 103. 19. EDw. glean. Tab. 247 .

127. Scarlattc: Buff. VII. p. 340. Cardinalis Briss. Ornith. III. p. 42. Tab. 3. I. Tanagra Brefilia, Livis. III. 2. Tyo Piranga, Ebiv. gle.rm. Tab. 343 .

1.27\% 2. Tangarn Scpricolor voyèz, pl. 7.1 .

128. I. Bec d'Argent Buff. VII. p. 365. Cardinalis purpureus Briss. Ornith. ilI. o. Tanagra Alliroftris, Linv. Ill. I 2 .

2. La Femellc.

129. Lagopede Burf. IV.p. $3^{3} 3$. pl. IX. Gelinotte blanche Briss. Ornitit. III. p. 49. Tetrao Lagopus, Linn. 103. 4. EDw. Biids. 72.

1 3 o. Gelinotte du Senegall. Buff. IIl. p. 361. Varieté de la pl. 105.

I 3 I. Gelinotte de Canada. Buff. III. p. 405. Bonafia Briss. Grnith. I. p. 205. pl. XX. 1. 2. Tetrao Canace, Linv. 103. 7.

iว2. La Femellc.

'33. 1. Le Jacou Buff. VIII. p. 406. Tangara punftata, Isin. Gen. III. 21. EDw. glean. Tab. 262.

2. L.e Rouverdin Buff. VII. p. 404. Tangara viridis pumatulata Buss. p. 23. pl. IV. 1. Tanagra Gyrola, Linv. IIl. 7. EDw. Birds Tab. 23 .

34. I. Foudi Burf. VI. p. 238 . Briss. Ornitit. O. LINN. o.

2. La Femelle.

135. 1. Durbec Burf. VI. p. 184. Grosbec de Canada, Burf. Omith. III. p. 250. pl. XII. 3. Loxia Enucleator, Lins. 109. 3. EDw.
Birds. 123.

2. Toui-nam Corvi Buff. VI. p. 195. Grosbec des Philippines BRtss. Arnith. Ill. 1. 238. pl. 12. I. Loxia Philippina, Linn. Geni, 109. $3 c$. 
Pl.

\section{(9)}

136. Perdrix de Montagne. Buff. IV. 195. Briss. Omith. I. p. 226. Tetraa Montana mihi. Linn. 103.0.

137. Bis-ergot Buff. IV. p. 23 I. Briss. Ornith. I. p. 23 I. T. XXIV. I. LinN. Gen. 277. I5.

1 38 . Pigeon verd des Philippines. Buff. IV. p. 356. pl. I I.

I 39. 1. Jacobin (Grosbec) Buff. VI. p. 199. pl. 16. Coccothrauftes Javenfis ianevia, Briss. Ornith. III. p. 239. Tab. I 3. 2. Loxia punfulata, Linn. Ge1?. 109. 18. Edw. birds 40.

2. Le Mâle.

3. Varicté du même.

i fo. Tourtelette. Burf. IV. p. 369. 4. Pigeon du Cap. Brrss. Ornith. I. p. I 20. Tab. 9. ^. Columba Capenfos, Linn. Gen. 1 o4. fp. 39.

141. Pigeon de Martinique Buff. IV. p. 354. Briss. Omith. I. p. 129. Tab. 12. 1. Columba Martinica, LinN. 104. 24.

142. Turvert Burf. IV. p. 397. Tourterelle verte d'Amboine Briss. Ornith. I. p. 152. pl. 1 5. 2. Columba viridis, Linn. Gen. 104. 23 .

143. Lory Perruche verd et rouge. Buff. XI. p. 366. Pfittaca coccinea infltlarmm bon. fort. Briss. Ornith. IV. 173? Pfittacus Borneus, Linn. Gen. 45. 11? Indian Lory. Latham birds I. p. 207.19.

i t4. Perriche a Gorge variée. Buff. XI. p. 366 . Briss. Ornith. IV. o. Yfittacus lyanopterus, mihi LinN. 45. O. Waved breafted Parrakeet, Lath. burds I. p. $245 \cdot 44$.

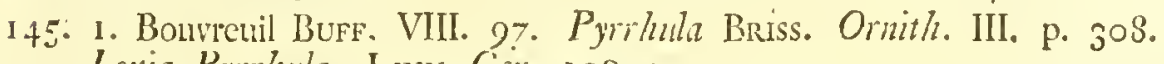
Loxia Pyrrhula, Linn. Geil. 109. 4.

¿. La Fcmelle.

146. Le Karraca (faifan.) Burf. IV. I 14. Briss. Ornith. o. Plafianus Katracu, Linn. Gell. 101. o.

147. Le Francolin. Burf. IV. p. 223. Briss. Ornith. I. p. 245. pI. XXIII. 2. Tetrao Francolimis, Livn. Gen. 103. IO. EDw. glem. Tab. 246.

I 48. Varicté du même.

149. Le Colinicui Burf. IV. p. 296. Caille de la Louifanie, Briss. Ornith. I. p. 258. pl. 22. Tetruo mexirants, Linv. Gen. 103.14.

150. La Bartavelle BuFf. IV. p. 197. pl. 17. Perdix graca, Briss. Ornith. I. p. 24 1. pl. XXIII. 1. Tetrio rufus, Lins. Gen. 103. 12. Edw. bira's 70 .

I5 I. I. Linotte Buff. VII. p. 85. Linaria rubra major, Briss. Ornith. III. p. 135. Fringilla canabina, Lins. Gen. 112. 28.

151. 2. Le Sizerin Buff. VIJ. p. 302. Linaria rubra minor, BrIss. Omith. III. I 38 . Fingilla Linaria, Linn. I I 2. 29.

152. 1. Le Padda, ou Oifenu de Riz, male, Burr. VI. 192. Rriśs. Orwith. III. p. 244. pI. XI. 4. Loxia Oryzivora, Linn. 109. I 4. EDw. birds Tab. 41 .

2. Le Flavert. Buff. VI. p. 191. Grosbec de Cayenne, Briss. Ornith. III. p. 229. pl. II. f. 3. Loxia flavicans, Linv. 109. I 3 .

153. 1. Domino Buff. VI. p. 200. Briss. Ornith. III. p. $23 \%$ pl. XIII. 2. Loxia punctulata, Linn. 109. 18. Edw. birds I. Tab. 40.

2. Le Rofegorge Burf. VI. p. is8. Grosbec de Ia Louifanic, Briss. Omith. III. p. 247 pl. XII. 2. Loxia Ludoviriana, Linn. I09. $3^{8}$. 
P1.

154. Grosbec bleu d'Amerique. Buff. VT. p. i 83 . Briss. fuphl. p. 89. va. rieté du Loxia Coccothraufies, Linn. voycz pl. 99. et 101.

I55. I. Tangara bleu BufF. VII. Ornith. III. p. 9. pl. 1. 4. Tanagra brafilienfis, Linn. 1 I 1.15.

2. Rouge Cap. Buff. VII. 377. Cordinalis Americanus, BRIss. Juppl. p. 67. pl. IV. f. 4. Tanagra gularis, LinN. Gen. I I I. I 3 .

156. I. Tangara du Camada. Bufr. VII. 350. Cardinalis Canadenfis, Briss. Ornith. III. p. 48. pl. II. fig. 5. Tanagra mutra, Linn. Gent. III. 3 .

2. I'Efchre Buff. VII. p. 37. T. Tangara Dominicenfis, BrIss. Ormith. III. p. 37. pl. II. f. 4. Tanagra Dominica, Linn. Gen. I I I. I6.

157. I. Senegali. Buff. VII. I 4 I. le Senegali rouge, Briss Ornith. III. P. ฉо8. pl. X. f. 2. Fingilla Senegalli, Linn. Gen. 112.18.

2. Wax bill ou fenegnli rayé. Buff. VII. p. I44. Bruss. Ornith, III. p. 210 . pl. X. f. 5. Loxia Aftild, Linn. Gen. 109. 2 I. Edw. birds Tab. 1-g. glean. 354 .

3 Olivette. Buff. VII. p. 205. Briss. Ornith. III. pl. VII. 2. finingilla finica, Linn. I I 2 . 20 .

158. I. Ortolan de la Louifanic. BuFf. VIII. 32. Hortulanus Ludoricianus, Briss. Ornith. III. p. 278. Emberiza Ludoriciana, Linn. Gen. I Jo. 10.

2. Ortolan du Cap. Buff. VIIT. p. 36. Hortulanus Capitis bont Spei, Briss. Ornith. III. p. 280 . pl. XiV. 4. Emberiza Capenfis, Linn. Gen. 110.9.

159. I. Le Pape. BUff. VII. P. 247. Chloris Ludoviciana, Briss. Ornith. III. p. 200. pl. VIII. 3. Emberiza ciris, Linn. Gen. I10. 24. Edw. Birds. pl. i zo. Catesb. Carcl. I. pl. 44.

159. 2. La Femelle.

160. Tourterelle du Senegal. Butr. VI. p. 394. BrIss. Ornith. I. p. 122. pI. X. f. I. Turtur afra, Linn. 104. 3\% \%

161. Varieté du même, a Collier.

163. Troifieme varieté du Pigeon commun.

I64. Pigeon ramier des Molucques. Buff. VI. p. 372 . Briss. Ornith. I. p. 1 48. pl. XIII. f 2. Columba anea, Linn. Gen. 101. 22.

I65. Spatule d'Amerique. Bufr. XIV. p. 2 I 4. Platea.rofea, Briss. Orniti. V.p. $35^{6}$. pl. XXX. Platalea Ajaja, Linn. Gen So. 2.

I66. Grigri (Toucan) Bufr. XIII. p. I88. Tucana Braflienfis siridis, Briss. Omith. JY. p. 4-6. pl. XXXIII. 2. Ramphaftos Aracuri, LinN. Gen. 462 Latinam birds s. p. 332. Io.

167. Perriche Pavouane. Bufr. XI. p. 357. Pfattaca Guijanenfis, Briss. Ore nith. IV. p. 33I. Pfittacus Pavia, Lins. Gen. 45. 0. Pavouane Parrot. Lathais bords 1. p. 232.34 .

168. Lory tricolor. Buff. XI. p. 183 . Lorius Philippenfis, Briss. Ornith. IV. p. 2ะ6. pl. XXIII. 2. Pfittacus Lory, LinN. Gen. 45. 27. EDw. IV. 'Tab. I70. Black Capped Lory, Lathasi I. p. $273 \cdot 78$.

169. I'Agami Burr. Vill. p. 259. Phafumus Antillurtun, Briss. Ornithi I. p. 269. Ifophiu crepituns Linn. Gen. 94. 1. Vosm. monogi:

170. Caille Burf. IV. p. 239. pl. 7. Perdix Coturnix, Briss. Ornith. I. p. 247. Tetrao Coturnix, Lins. Gen.103. 20. 
Pl.

\section{( II )}

17 1. Tumix Beff. IV. p. 294. Briss. Ornith. I. o. Linn. Gen. 103. o. 172. Le petir Tetras. Bufr. III.p. 302. pl. VI. Lagopus Urogallus minor, Briss. Ornith. I. p. 186. Tetrao tetrix, Linn. I03. 2.

173. La Femelle.

174. Tourterelle de ha Jamaique. Buff. IV. p. 402. Briss. Ornith. I. p. I 35. Tab. XIII. I. Columba Cyanocephala, Linn. I +4. 20.

175. La Tourte. Buff. IV. 4OI. Tourterelle de in Caroline, Briss. Oraith. I. p. VIII. fo I. Columba (Turtur) Carolinenfis, Lins. 104. 37.

${ }_{17} 6$. Tourterelle de Canada. Buff. IV. p. 393. Briss. Ornith. I. p. I I \&. Columba Canadenfis, Linn. 104. 30.

177. Turvert Buff. IV. p. 397. Briss. o. Linn. 104. O. Columba Turtur yiridis, mihi.

178. 1. Le Bluet. Buff. VII. 374. Eveque de Cayenne, Briss. Omith. III. p. 40. Tab. I. f. 2. LiNN. Gen. o.

2. La Femelle.

179. I. Turquin Buff. VII. $3^{6} 3$. Tangara bleu du brefil, Briss. Ornith.lli. p. 9. pl. I. f. 4. Tanagra Brafilienfos, Linn. Gen. 1 44. 15. Os varieté de 155 .

2. Tangara noir Buff. VII. p. 361 . Briss. Ornith. III. o. Tangaria Altiroftris, Linn. II 1. 18. (Voyez la femclle pl. 711.)

180. Gorgenue (Perdrix.) Buff. IV. p. 233. Briss. Orint/2. o. Linn. 103. o. Tetrac undicollis mihi.

IS I. I. Friquet hupé. Buff. VI. p. 233. BRiss. Ornith. III. o. Linn. O. Palfer Criftatus mihi.

2. La Fcmelle.

182. Merle de Montagne. Burf. VI. p. 16. Briss. Orwith. p. 232. pl. XXI. I Merula montana, LINN. Jy/t. VI.

183. I. Pere notta Iongue quene. Buff. V. 226 Pafer niger, Briss. Ornith. III. p. 1 18. n. 23. Tab. 7. 1. Fringilla Noetts, Linn. Gen. II 2. I9.

2. Moincat a bec rouge. Buff. VI. p. 223. Briss. Ornith. III. p. I 10. pl. VI. 1. Lns. Gen. i IO. S. Emberiza Quelea.

184. Yapou. Buff. V. 323. Casficus luteus, Briss. Ornith. II. p. 100. Tab. 9. fig. I. Oriolus Perficus, Linn. Gen. 52. 7. Bhack and yellow Oriole Lath. Birds I. p. 4I8. I.

185. I. Traquet des Philippines. Ruff. IX. p. 33. Ruhetia Philippenfis, BrIss. Ornith. III. p. 44+. pl. XXIII. 2. Motaculla fulicata, LiNs. Gen. 11 4. 39.

2. Grand Trnquet des Philippines. Buff. IX. p. 331. Briss. Orinth. III. p. 44.6. Motacilla Violacea. mihi Linn. Gen. i I 4. o.

I S6. Cordon bleu (Cotinga). Bufr. VIII. p. 194. pl. S. Cotinga Briss. Ornith. II. p. 340. Aimpelis Cotinga, Lann. 108. 4.

187. Ouroubou. Buff. I. p. 246. Vautour du Brefil, Briss. Ornith. I. p. 468. Vultur Aura, Linn. Gen. 41. 5. Carrion Vulture, Lath. Birds I. p. n. 5 .

188. Cordon bleu. (Femelle) royez pl. 186.

189. Drongo (Gobemouche.) Bufr. VIII. p. 413 . grand gobemouche noir hupé de Madagascar, Briss. Ornith. II. P. 388. Muscicapa gulleata, LINN. II 3 . 0 . 
P1.

190. I. Toui: Perriche a gorge janne. BUFF. XI. p. 390. Pfittacula gutture luteo, Briss. Ornith. IV. p. 396. pl. XXX. f. 3. Pfittacus Flavi. gula, LinN. 45. o. yellow Throated Parrakect, LATir. Birds. I. p. 319.11. 128.

2. Perruche a tete bleue. Burr. XI. p. 228. Pfittacula Philippenfis, Briss. Ornith. IV. p. 392. Tab. XXX. fig. 1. Pfittacus Galoulus, Linn. Gen. 45. 46. Sapphire Crowned Parrakeet, Edw. gleuti. I. 293. I. LATH. birds 1. I 19.

191. Petit Cacatoe a bec Couleur de chair. Buff. XI. p. 133. Cilcatua minor, Briss. Ornitll. IV. p. 21 2. Linn. o. red veuted Cokatoo, Brown Illuytir. pl. 5. LATH. Birds I. p. 258. n. 63.

192. Perruche a tête bleue et queue longuc et egale. Buff. XI. p. 20 I. P/it . tacus Cyanocephalus, BRIss. Ornith. IV. p. 359. Pfittacus Cyanocephallus, Linn. 43. 10. Blue lieaded Parrot. Lath. Birds. p. 21 I. I3.

19:. Engoulerent d'Europe, Buff. XII. p. 2 I 6. Tete chevre BRIss. Ormith. II. P. 470. pl. XV. Caprimulgus Europaeus, Linn. Gen. 118.1.

194. Veuve a Collier d'or. Buff. VII. p. 221 . lu veuve Briss. Ornith. III. p. 120. pl. VIII. I. Emberiza Paradifea, Linv. Gen. 43. 10.

195. Couroucou a ventre Jaune. Burf. XI. p. 407. Trogen Cayanenfis viridis. Briss. Ornith. IV. p. I68. pl. XVII. 2. Trogon Carucui, Lin, Gciz. 55. 2. yellow bellied Currucui, LATH. birds I. p. 489.

196. Pic varié male. Buff. XIII. p. 85. Epciche BrIss. Ornith. IV. p. 34; Picus major Linn. Gen. 59. I I. great fpotteid woodpecker. L.AtiI.

197. La Foalque. Burf. XV. p. 327. pl. I 8. Fulica Briss. Ornith. VI. 23. pi. Il. f. i. Fulleca atra, Linn. 91. 2.

19S. Courly. Bufron XV.p. 38. Numeninus Madugascarienfis, Briss. Or. nith. V. p. 321. Tab. XXVIII. Scolopax Miadigascirionfos, LinN. 86. $=$

199. Jaunoir (Merle) Burf. VI. p. 52. Merle du Cap. de Bonne, Esperance Briss. Ornith. II. p. 309. pl. XXIII. 2. Turdus morio LiNs. 107.26.

200. Goulin Buff. VI. p. I 3 I. Merle chauve Briss. Omith. II. p. 28 . Gracula calsa, Linv. 53. 2. Bald gracle LATHAm birds 1. p. 4.3."

201. I. Pere noir de la Martinique. Buff. VI. 226 . Brisson Ornitit. IIi.o. Linn. Gen. 11z.o.

2. Pisfevert Buff. VI. p. ${ }^{2} 36$. Briss. Ornith. III. p. i i 8. pl. Vil. fig. 1. Fringilla Noitis, Linn. Gen. I I 2.49.

202. 1. Serin des Canarics. Buff. VII. 1. Briss. Ornith. III. I8 4. Fringillı Camarienfis, Linn. Gen. I1 2.23 .

2. Chardonneret janne. Bufr. VII. p. 297. Carduelis Americum, Briss. III. p. 64. Fringillat triftis, Linn. Geul. 112.12.

203. 1. Le beau marquet. Buff. VI. p. 239. Briss. Ornith. III. o. Fringilla Speciofa, mihi Linn. Gen. I I 2. o.

204. I. Le Bouverec. Buff. VIII. p. I 18. Briss. Ornith. III. o. Loxia Nigro aurantia, Linn. Gen. I09. 0.

2. La Femelle.

205. J.e grand Tangara. Burr. VII. p. 334. Tangara major BRIss. Ornith. 111. o. Tamagrit. ..... Linn. I 14.0 .

206. 1. Timnatia a tece et Gorge rouge. Buff. XIIJ. p. $1430^{\circ}$ Buiso Coryanchhis, Briss. Ornith. IV. p. 95. pl. VII. f. I. Butro Erythroceplealus,
Linv. 56. o. 
2. Varicté du même. Brass. Ornit/. IV.p. 97. Caycnne barbet Lath. birds p. 495. 2 .

207. Harle huppé BufF. XV. p. 423. Merganfer criffatus, Briss. VI. p. 237 . Mergus Serrator, LinN. 68. 3.

208. Femelle de l'Eider. Brıss. Ornith. VI. p. 494. pl. XX.

209. l'Eider (Canard) Burf. XVII. . . . . A Alfer lanuginofus, BrIss. VI p. 494. Tab. XIX. Anas mollisfimu, Linv. Gen. 67. I5.

210. Gerfaue Buff. I. p. 339. pl. I 3. BRiss. Ornith. I. p. 370. 373. pl: XXXI. Falco Gyofulco, Linvo 42. 27. Brown Jer falcon Lath. Birds I. p. 82. 11. 68.

211. Coucou Pinye, Buff. XII. I S. Coucon de Cayenne, Briss. Ornith. IV. p. 122. pl. VIII. f. 2. Cuculus Cajanius, L.inn. 77.14.

21 2. Bentaveo Burr. VIII. p. 4O1. Tyran du Brefil, Briss. Ornith. II. p.401' pl. XXXVI. 5. Lanius Pitangua, Linv. Getı. 44. 15. Brafilian Shrike JathaM birds I. p. 87. n. 39 .

21 3. Ramiret Buff. IV. p. 376. Briss. Ornith. I. o. Lins. o.

2 i 4. Varicté du Turvert. Burf. IV. p. 398.

2 5 . Perruche a double Collier Burf. XI. p. 199. Briss. Ormith. PJittaca Borbonica torquata, Pfittacus eques mihi Linn. Gen. 45. o. double ringed Parrakeet Latuam bird's I. p. 236. 37.

216. Lori Noira Buff. XI. p. 1 76. Lorius moluccenfis, BRiss. Ornith. IV. p. 219. Pfittacus Garrulus, Linv. Geino 45. 25. Scarlet Lory, Lath. Birds. I. p. 270. n. 76.13 .

217. Le Phaifan tricolor BuFF. IV. 10I. Phaím doré de la Chine, Briss. 21\% has fige. 1.82 2. Orvith. I. p. 271. Phafianis pictus, Linn. Gell. 101. 5. Edw. birds Tab. 68.

218. Le bec Croifé Buff. VI. p. 172. Loxia Briss. Ornith. III. p. 329. pl. XVII. fig. 3. Loxial curvirgfira, LinN. IO). I.

219. Martin Bufr. VI. p, I 35. Merle des Philippines Briss. Ornith. II. p. 278. Paradifear Triftis, Linv. Gen. 53 3. 3aradifi gracle Lathas birds I. p. +59 .

220. Le verd doré Buff. VT.p. 57. Merle a longue queue, Briss. II. p. 313 . Turdus longicauda mihi LinNo 0.

בz I. Oran bleu Burf. VI. 69. Merle brun du Cap de b. E. Briss. Ornith. I1. p. 259. pl. 273 ? Turdus Capenfis, Linv. 107.17?

222. Cuille des Isles Malouines Buff. IV. p. 28 1. Briss, Ornith. o. Tetrao Malouinus mihi Linv. o.

22.3. 1. Moineau du Senegal Bufr. VI. p. 222. Fringillı Senegalla, Linv. Gen. 11 2. 18. Senegali Briss. Ornith. III. p. 208. pl. X. f. 2.

2. Soulciet Burf. VI. p. 245 . Moineau de Canada. Briss. Ornit/2. III. p. 102. 15. Fringilla Canadenfis, LiNn. II 2.0.

2243 Jacarini Burf. VII. p. 4 I2. Tangara noir du Brefil BRsss. Ornith. III. p. 28. Tangara Facarina, Lins. III. 4 .

225. La foulcie Burf. VJ. p. 240. Moineau des Bois, Briss. Ornith. III. $224 . f 2 q 1$ meite
frg. 2 meite p. 38. Tab. V. f. I. Fi ingilla Petronia Linn. Gen. I I ב. $3^{\circ}$

226. Choucas Mouftache Buff. V. p. 109. Briss. Ornith. II. p. 33. pl. II. f. 2. Corrus Hottentottus, Linn. Ger. 50. I. Hotentol Crow. Latis. kirds p. 38010 .

227. I. Oifeau mouche huppé, Buff. XI. 49. Mellifuga criftata Briss. Or- 
1.

nith. III. p. $41 \%$ pl. 37. 2. Trochilus criftatus; Lins. Gen. 66. 18. Colfted. green Humming bird EDw. birds I. 37. Latiı. birds I. 783, n. 53 .

2. Rubis Topaze. Burr. XI. 26. Mellifaga Brafilienfes gutture Topazino, Briss. Omith. III. p. 699. pl. 37. 1. Trachilus Mosquitus, Linn. 66. 14. Ruby necked Humning bird. Lath. lirds I. p. 779. 1.. 46 .

228. Becardé vanga Buff. II. p. 93. Piegriefche Briss. Ornith.II. o. Lanius Cetrvirofiris, Linn. Gen. 45. 8. hook billed fhrike, Lath. birds I. p. 171. n. 19.

229. Co:inga a plumes foycufes Burr. VIII. p. 201. Cotinga Mainas Briss. Orinith. Il. p. 34 I. pl. XXXIV. 2. Cotinga maynana, LinN. 108. 5.

=30. i.Le Croisfant. Buff. VII. p. 245 Briss. Orkith. III. p. 10 \%. pl. V. f. I.Linn. Gen.II2? o.

2. Le Petit Moincenu du Senegal Burf. VII. p. I 49. Pasfei Briss. Oimith. Ill. o. Fringilla rofea, Linn. 112. 0.

3. Le ferevan Buff. VII. p. 147. Pasfer Briss. Ormith. III. o. Fringilli: Commeijonia, mibi Linn. Gen. I I2. o.

231. La Bartavelle (Perdrix) Burf. IV. p. 197. Perdrix rouge Briss. Oimith. I. p. 241. pl. XxIll. fig. 1. Tetrao rufus, LinN. Gen. 104. I 2 。

232. Martin pecheur bleu ct roux Burf. XIII. p. 267. Ippida Madigasiavienfis coerulea, Briss. IV. p. 496. pl. XXXVIII. 1. 2. Aliedo Smyrnenzis, Linv. Gen. 62. n. I I. S. great Gambia kingstilher Edw. bird's I. S. Lath. Birds I. 616. 7. A.

233. Le Proyer Buff. VIII. p. 73. Cynchiramus Briss. Ornith. III. p. 292 Emberiza miliaria, Lins. Gen. I 10. 3 .

34. 1. Moticherolle huppé a tete Couleur d'Azur Buff. VIII. p. 36-.Tab. 4I. Briss. Ornithlil. o. Linn. o.

2. Muficapa criftata alba Cap. Bon. fpei Briss. Ornith. II. p. 4 ז. pl. 41. 2. Mufcicapa Paradisi, Linn. II 3. I. Low. lirds il 3 .

235 has fugs 1.92 2. 235. Traquec de Lucon BurF. IX. p. 329. Rubetra Luconienfis, Briss. Cirnith. III. p. 442. pl. XXIV. fig. ^. 3. Motaillat caprath, Linn. Get:。 I I 4. 33 .

z j6. I. Le Siffleur Bupr. V. p. $3^{I 6}$. Baltimore verd. BRIss. Ornith. II. p. 113. Oriolus Baltimore, Linv. Gen. 5\%. 10. B. Wifler Oriole LATH. I. p. 443. n. 34.

2. Valricté du Commandeur voycz pl. $40 \Omega$.

23.- Le Albatros Buff...... le Albatros Briss. Omith. VI. p. 126. Diomedea exulans, Linn. Gen. 7. 1.

238. Jacimare Bufr. XIII. p. 325. Galbula Briss. Omith. IV. p. 86. pl. V. fig. I. Alcedo galbuta, Linv. Gen. 62. n. 15. green Jacanar Latu. birds p. 603. I. Jacamaciri Edw. glean pl. 334. Cupreous Jacanar PEN. Gerl. of birds p. 60. pl. 3 .

239. Grande Perruche a ailes rougeatres Burf. XI. p. 2 1 o. Pfittaca Ging itana Briss. Ornith. IV. p. 31 3. Pfittacus Eupatria, Linv. Gek. 4.5. \% Gingi Perrot LATH. I. p. 209. 10.

₹10. Lori Perruche tricolor Buff. XI. p. 192. Pfittaca Amboinemis coccinea Bisiss. Ornith. IV. pl. XXVIII. f. 2. Pfittacus Ambioinenifis, Livx. Gicus. 45. \%. Amboina red Parrot, Latham birds I. p. 2 10. 11, In. 
P1. .

2 千1. La Demoifelle de Numidie. Buff. XIV. p. 8. Grus Numidica; Briss. Ormith. V. p. 388. Ardea Virgo, Linn. Gen. 84. 2.

2.4. Le Vanneau. Buff. XV.p. 76. Vanellus Briss. Ornith. V. p. 94. Trina ga Vanellits, Linn. Gen. 87.2.

=43. Le Cocotzin (Pigeon) Burr. IV. p. 4I 3. perite Tourterelle de Martini- 243. has fegs. / 2 2 que Briss. Ornith. I. Tab. IX. fig. 3. Columba Pasferina, Linn.Gen. 104. 34 .

244. Tourterelle des Indes. Buff. IV. Briss. Ornith. I. o. Linv. 104. o. NB. Monfieur de Buffon ne la cite pas, dans fon hiltoire des oifeanx.

245. Outarde male Buff. III. p. 1. pl. I. Ontarde Briss. Ornit/s. V. p. I8. Otis turdal Linn. Ger. 95. 1.

246. I. Souimanga marron pourpré. BufF. X. p. 247. Grimpereau pourpré des Philippines, Briss. Ornith. III. p. 655. pl. XXXI. f. 2. 3. Certhia fperatu, Linn. Gen. 65. 13. red brealted Creeper Lathunt 706.

2. Sa Femelle Briss. 1. c. fig. 3 .

3. Souimanga a Collier Buff. X. p. 254. Grimpereau a Collier Briss. Ornith. III. Tab. 32. 1. Certhia chalybea Linn. Gen. 65. 10. Parple Indian Creeper Edw. glem 265. Collared Creeper LaTH. birds 709. 10.

247. 1. Ortolan Buff. VIII. i 3. Briss. Orwith. III. p. 269. Emberiza hortulanus, Linn. Gen. I I O. 4 .

2. Ortolan des Rofeaux Buff. VIII. p. 19. Briss. Ornith. III. p. 274. Emberiza Schoeniclus, Linn. Gen. 1 10. 17.

248. I. Schet all (Moucherolle) Buff. VilI. p. 383 . Gobemouche varié a longue queue de Madagascar, Briss. Oinith. IL. p. 420. Mufcicapa siridescens, milhi LinN. 113. o.

2. Le fchet vouloulo!ı. Buff. ibid Gobemouche a longue queue blanche, Briss. Omith. II. p. 427.

249. Le Gurlu. Burs. V. p. 164. Brisson Ornith. II. o. Corrus Leuiogaster milhi Linn. Gell. 50. O. yellow Bellied Gay, Latham berds I. p392. 28 .

250. Merle bleu Bufr. VI. p. $3^{6}$. Merle folitaire Bruss. Ornith. II. p. $28 z$. Turdis cyates, Linn. Gen. 10\%. 11. =4.

251. Merle Couleur de Rofe Buff. VI. p. 27. Briss. Ornith. II. p. 250. Turdus rofeus, Linn. Gen. 107. 15 .

252. Guepier Marron et bleu Burf. XII. p. I 90 . Guepier de l'Isle de France, Briss. Ornith. IV. p. 5+3. pl. XLIV. 2. Merops bicolor, Linn. Gen. 63. 0. Chesnuth bec-cater Lath. birds I. p. 677.9.

253. Gocland a Manteau gris Bufr. XVI. p. I 89. pl. 8. Larus cinereus, Briss. Ormth. VI. p. 160. Larus Cinereus, Linv. 76. 3. ( $\beta$ ).

55 . Oifeau de Paradis Buff. V. p. $20 \%$. Briss. Ornith. II. p. I 35. Paradisea apoda, Linn. Gen. 54. I greater bird of Paradile EDw. I 10. Latham birds I. p. 47 I. I.

55. Crave (Corbeau) Bufr. V. p. 2. Tab. I. Coracius Briss. Ornith. II. p. 3. 1. I. fig. 1. Corvus graculus, Linn. Gen. 50. 18. Red legged Crow. Lath. birds I. p. 401. 11. 39.

856. Storne Buff. V. p. ${ }^{2} 6_{3}$. Sturnus Ludovicianus Briss. Ornith. II. p. 449. Tab. IV. Sturnus Ludovicianus, Lins. 106. 3. 
P1.

25\%. Breve Buff. VI. p. I23. Turdus (Grive) Briss. Ornith. O. Turdus maciounus fasciatus, Livn. 10-. 0. Chort tailed Clow. Latu. lirds I. p. 398. n. $37 . \mathrm{c}$.

258. Breve de Bengale Bufr. VI. p. I 23. 3. Merle vert de Bengale Drsis. Ornith. II. p. 3 I 6. Merula (Corvus) Brachymins, Linv. Gen. 50. 15. Thort Tailed Crow. Latil. birds I. p. 398.

259. Le Paterich (Guepier) Buff. XII. p. 193. pl. XLII. 1. Guepier de Nadagascar, .Briss. Ormith. IV. p. 545. Merops Superriliofus, Linn. Givil. 63. 4. Merops Perfica Pallas Reiz II. append. n. 16. Tab. D. Sijpercilious bec-cater LATH. p. 673.4 .

¿60. Le Tock Buff. XII. p. 209. Hydrocorax Senegalenfis, meluno rynclam Briss. Ornith. IV. p. 573. pl. II.VI. I. Buceros mafutu, Liñ. 4:4. Red billed Hormbill, LATH. birds I. p. 355. n. Iо. A.

26i: Le Jafeur Burf. VI. p. 144. Bombycilla Bohemica Briss. Ornith. Il. p. 333. Ampelis Grurrulus, Lanv. I08. I.

262. Toucan a Gorge blanche Buff. XIII. p. 181. Tucana Guajane ujis gitnue albo, Briss. Ormith. IV. p. 416. Ramphaftos Hiscivorus, lix... 151. 4. Ieft Baralian Pie Euw. lards pl. 64. Lath. lirds p. 32\% +. Totican.

26́3. Kakatoës a huppe blanche Buff. XII. p. I 28 . Briss. Omilla. IV. F. 21 4. Pfittacus criftatus, Linn. Geu. 45. 22. (B) grcat IVhite Coukaţoo Lath. lirds I. p. 256. n. 61. EDw. birds 160.

264. Perruche a tete rouge BuFf. XI. p. 200. Pfittaca Erythrocephaha gingiana Briss. Ornith. IV. p. 346. Lins. 45. o. Blosfom liended l'amber LATH. birds I. p. 239.39.

265: Oifanu Royal Buff. XIV. p. I5. Tab. II. Ardea Balearica Biriss. O:nith. V. p. 51 I. Aidea Pavonina Lins. Gen. 84. I.

266. Goëland varié Buff. XVI. p. 200. Tab. IX. Briss. Omith. VI. 19. I6?. Linn. Gen. 76. o. Larur Maculatus mihi.

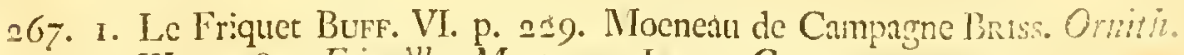
III. p. 82. Fringilla Montand, Linv. Gen. $112.3 .$.

2. Le Verdier Buff. VII. p. 240. Chloris Briss. Ornith. I!I. p. 190. Loxia Chloris Linn. 109. $2 \%$

¿68. I. Mainate Buff. VI. p. 125. pl. VI. Briss. Orinth. II. Tab. 28. f. 2 . Gracula religiofa Loinn. Gen. 53. 1. Lesfer Mino Enw. 1. pl. 11. Minor gralile Latham birds p. 455. 1.

269. Toucan a Gorge Jaune Buff. XIII. p. I 8 I. Male de $26 \simeq$. Rarmplaghins discolor Lin. Gen. 46. 7. yellow throated Toucan Latuam litids I. p. 325. 2 .

270. Beccasfine du Cap. Buff. XIV. p. 269. Briss. Juppl. p. 1 41. pl. VI. Scolopax Capenjis Lins. Gen. 86. 14.

2.- I. Jacamar a longue queue Buff. XII. p. 327. Galbula iongicanda Briss. Oinitl. IV. p. 89. pl. V. 2. Alcedo Paradifea, Lins. 6.. 1 t. Lög tailed green Jacamar Latı. Birds I. p. 605. pl. 36. 1. A.

$\approx 72$. Le Plaltron noir Buff. VI. p. 64. Merle a Collier du Cap. Bisiss. Ornith. II. p. 299. pl. XXX. I. Turdus Ceilonicus, Lixx. Gen. 10-. 28. the green Pie of Ceilon EDw. glean Tab. 32 I.

273. 1. Merle olive Buff. VI. p. 107. Merle de St. Domingo, Bruss. Ornith. Il. 1..396. Turdus virens, Lins. Gen. 107.0. 
2. Terat Boulan (MIric) Burr. VI. p. 98. Meruliz Olinacea, Briss. Omith. II. p. 248. Turdus Terat, Linv. 107. 0.

274. I. Coukeel (Coucon) Butr. MII. p. 38. Coucou noir des Inies Briss.

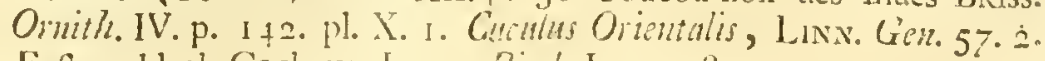
Entern black Cockow. Latil. Birds I. p. 5 I 8.10.

2. Coucon huppé a Collier Burrox Xit. p. 46. Coucou huppé de Coromandel BRIss. Ornith. IV. p. 147. pl. X1. 1. Cuculus Coromandus Linn. Gen. 57. 20. Collared cucliow, Latr. birds 1. p. 529. 26.

275. Le Macareu Buff. ... Fratercula Briss. Oinith. VI. p. 81. pl.VI. 2. Afia Aitica, Linv. Gen.69.4.

276. I: Le plus petit oifenn Monche Bufr. XT. 1 +. Mellifuga Briss. III. p. 697. pl. XXXVI. 1. Trochilus minimus, Linv. Gen. 66. 22: leaft Humming bird LaTH. Birds. I. p. 788. 60. EDw. birds II. Tab. 105 .

276. 2. 5 Oifeau Nouche vert doré. Buff. XT. p. 39. Mellifuga Cayanenfis 3. Briss. Ornith. III. p. ZO4. Mellifuga Dominicenfis, ilid 70z. Trochilus Mellijugus, LinN. Gen.66. I5.

4. Oifeau Mouche Rubis Emeraude, Buff. XI: 4.3. Mellifusa Brafilienfis, gutture mutro, Briss. Omith. III. p. 706. Trochilus rubircauda, Linn. 66. 0 .

27. Coucou varié de Mindanao, Burf. XII. 26. Coucou tacheté de Mindanao Briss. Ormith. IV. p. I 30. Cuiculus Mindanenfis LinN. 57. 3. Mindanao Cuckow, Latil. birds I. p. 515. 5 .

-78. Four de Basfan Bufr...... Sula Basfana, Briss. V. p.503. pl. XLIV. Pelecanus Busfanus; Lisv. 72. 5 .

279. Pacäpac ou Pompadour (Cotlnga) Buff. VIII. 20.3. Cotinga pourpré Briss. Omith. II. p. 347 pl. AXIV. I. Ampelis Pompadora, Lins. 108. =.

280. Etournenu Pic Burf. V. p. 26 r. Etourneau du Cap de bonne Esperance Briss. Ornith. II. p. 446. Sturnuts Cateutfis, Lins. 106. 2.

28 r. Pic rayé de St. Domingo Burf. XIII. 39. Briss. Omith. IV. p. 65. Pisus Striatus, LinN. O.

282. I. Coliou Buff. VIII. p. I +2. Colius Capitis bone Spei, Briss. Ornith. III. p. 304. Loxial Colius, Linñ. Geil. 102. It.

2. Coliou huppé Burron VIII. P. 143. Colius Senegallenfat criftatus, Briss. Ormth. III. p. 306. Linn. Gen. 109. o. Low: Colius (B).

283. Calao des Moluces, Buff. XII. p. zis. Bydrocorax Briss Ornith. IV. p. 556. pl. XLV. Buceros Hydrocorat, Lisv. 47. 2. Indian Hornbill. Lath. birds I. p. 35 I. I.

284. l'Alatli (Martin pecheur) Buff. XII. p. 307. Ispida Mexicana criftata Briss. Ornath. IV. p. 518. Alcedo torquata, LinN. Gen.62.8.

285. Cuit (Rollier) Burf. V. p. 197. Rollier de Mindtunaö, Briss. Ornith. II. p. 69. Coracias Benghulenfis, LinN. 5I. 5. Bengal Roller Latr. hirds I. p. 4 IO. 5.

286. Pluvier a Collier de St. Doningo, Burf. XV. p. 153. Pluvialis Dominicen/is torquata, Briss. Oinith. V. p. 70 . pl. VI. 2. Charadiuts torquatus, Linn. Gen. 83. 8.

287. Perruche a Ailes chamarrées, Burf. XII. p. 2 Io. Briss. Ornith. IV.o. Pfittacus gala, mihi Linn. Gen. 45. o. Lace winged Parrakeet LatH. birds I. p. 244. n. 42 . 
288. Perroquet a tete Grife, Buff. XI. p. 171. Pfittaculat Sentgallenflis, Briss. Omith. IV. p. 400. Tab. XX11I. 2. PJittacus Sentegallus, Lisw. 45. 43. Senegal Parrot, Latr. birds I. p. 30\%. n. II 4.

289. Roi des Gobemonches BufF. VIII. p. 386. Mufricapa Briss. Omith. II. LinN, O.

290. I. Pasfevert (T'mgara) Büf. VII. p. 396. Tangara vert de Cayeme Briss. Ornith. II. p. 21. pl. IV. 3. Timagra Cayana, I.INN. I I I. 8. 2. Diable enrhumé BuFf. VII. p. $3^{82}$. Tungara Cayanenfis coemlea. Brtss. Ommth. III. p. 6. pl. I. fig. 3. Tumegra Mexicana, Linn. I I I, IO.

29r. I. \{ Pcre noir Buff. VI. p. 225. Briss. Ornith. VII. p. I 18. Pasfer ni2. $\{\mathrm{ger}$ ? confer et pl. 183. la Femelle Emberiza atrata, Linn. I Io.

292. I. Tarin de la nouvelle York, BuFf. VII. p. $3^{\text {i }}$. Ligurinus BrIss. Ornith. 111. p. 65. 3. Fringilla fpinas, Linv. I12. 25.

2. La Fcmelle:

293. Pique locuf Burf.V. p. 239. Tab. I 4. Buphaga Briss. Ornith. II. p. 437 . pl. XLII. 2. Buthaga Afracana, Linn. 48. I. African Bcef. catci, LATH. birds I. p. 359. I. pl. XII.

294. Cuil (Coucon) Buff. XII. p. 23. Coucon tacheté de Miabar, Brıss. Ornith. IV. p. 136. pl. XI. 2. Cuculus honoratus, Lins. Gen. 57· 7.

295. I. Toulon (Concou) Burf. XII. p. 2 I. pl. 2. Coucou de Mudagascar, Briss. Ornith. IV. p. I 38 . Cuculus Melanorynthos mihi Livn. Gen. $5 \div$. 0.

2. Taitfon Courou Burf. XII. p. 50. Tab. 3. Courou blen de Mirdagiascar, Briss. Ornith. IV. p. I56. pl. XIII. fig. I. Cuculus Coeruteus, Linn. Gen. 57. I 5.

-96. Bé-carde (Piegriefche) a ventre Jaune, Burr. II. p. 92. femellc cic 229 . yellow bellied fhrike, Latriam Birds. I. p. i 88. 40.

297. I. Picgricfche de fencgal Burf. II. p. 75. Briss. Ornith. II. p. I $6=$ pl. XVII. I. Latius Senegallus, Linv. Gen. 44. =1. Sencigulfhrike Lath. Birds I. p. I6z.6.

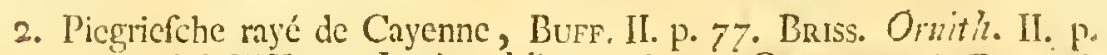
18\%. pl. XXIX. 3. Lanius doliatus, Linn. Gen. 44. IG. EDw.p1\% 226. Pied fhrike Lath. birds I. p. 190. 11. 44.

298. 1. Piegriefche blcue de Madagascar, BufF. II. p. 76 . varicté de 445 .

2. Schlat-bé (Piegricfche) Bufr. II. p. 94. Piegriefche rousíe de Madagascar, Briss. Ornith. II. p. I78. pl. XVIII. 4. Lamius rufus LinN. Gen 44. 17. LatiI. birds I. p. 180.31 .

299. I, Cali-Calic (Piegriefche) Butf. II. p. 97. Briss. Ornith. II. o. Lamius Madugascarienfis, Linn. Gen. 44. 22.

2. La femelle; Madagascar fhrike L.ath. birds I. p. 174. 24 .

\$00. Chevalier varié Buff. XIV. p. 305. Chevalier Cendré (Totanus Briss. Ornith. V. p. 203. Tringa littorea Lins. Gen. 8\%. 17.

301. I. Le Cyacou (Tangara) Bufr. VII, p. 406. Tungara viridis Indica punctulata Briss. Ornith. III. p. 19. pl. IV. Tanagra puntata, Linn. Gen. III. 2 I.

2. La Houpette (Tangara) Buff. VII. p. 3.36. Tamgara noir huppé, Briss. fupplein. p. 65. pl. IV. 3. Tarigra crijtata LinN. Gen. II I. $2 i$. 
รо2. I. Cisfe noifete (Alunkin) Bum. VIIT. p. 155. Mamakin Brsss. Or. nith. IV. p. 442. Pipra mubisits, Live. Gen. 115.12.

2. Manakin Orange, Burf. VIII. p. 159. Nanakin rouge Briss. Ornitlis IV. p. 452. pl. XXXIV. 3. Pipru Aureola, Linn. I I5. $\%$.

3०3. 1. Varieré de la Casfé noifete (302. 1).

2. Taýe jeune Manalin hupé noir, Brıss. Onzith. IV.p. 459. pl. 35. Pipra Pareola Linn. Gen. I15. 2. Manachs Superbus, Pall. fpicl. VI. Tab. 3.

NB. Mlonficur Burfos penfe que le manklin vert a crête rouge pl. 687 . 2. eft le même parfait.

504. Bacarde (nrale) (Piegricfihe) Buff. II. p. 91. Piegricfche grtife de Ca-

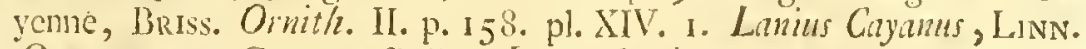
Gen 44. 20. Cayeme fhrike, LATH. burds I. p. 189.

305. Le Combattant, Burf. XIV. p. 31 I. Tab. XV. XVI. Combattant Briss. Ornith. V.p. 240. pl. XXI. Tring Plugax, Linn. Gin. 87. I.

3o6. La Femelle.

307. Toncan a gorge Jaune, Burr. XIII. p. 178. Briss. Ornith. IV. p. 419. pl. XXXil. I. Ramphaftus Tucamus, Linn. Gen. 46. 5. jellow brcaited Toucan Latri. I. p. 326. 3. EDw. pl. 329.

3०3. Lunme ou pecit plongeon de Mer, du Nord, Burf: XV.p. 405. Plongeon a gorge rouge, Briss. Ornith. VI. p. I I . Tab. XI. 1. Culymbus Sfftentrionalis, Linv. Gen. $75 \cdot 3$.

309. I. Le Grivelin Buff. VI. p. I 89. Grosbec de Brefil Briss. Ornith. III. p. 246. Loxia brafiliana mihi Linn. Gen. I09. o.

2. Rougenoir (Grosbec) Bufr. VI. p. 190. Briss. Ornith. VI. o. Lo. xia Linv. 109. 0.

310. I lirondelle a ventre roux Buff. XII. p. 352. Hirondelle de Senegal, Bnss. Ornith. II. p. 496. pl. 45. I. Hiruindo Jenegallenfis, Linn. Gen. 117.8 .

3i I. Le Jaco (Perroquet) Buff. XI. p. 38. Perroquet Cendré de Guinée, I3riss. Oruith. I\%. p. 3Io. Pfittais Enithatus, Linv. 45. 24. ashcoloured Parrot. Lathani Birds. I. p. 261.

3 Is. Perroquet vert et rouge de Cayeme; Buff. XI. p 295. Briss. Ornith. IV. o. Pfittacus Eupatria, $\beta$ Liñ. 4 t. 7 ? yellow Crouncd Parrot Lati. birds I. p. 282.89 . A.

313. 1. Cafoar Buff. II. p. 306. Briss. Ornith, V. p. XII. pl. I. 2. Stritthio cafuarius, Lins.Gen. 96. 2.

3 I . Varicté du Guepier marron ec blcu, de la pl. 252 .

315. Pcrit Butor Burf. XIV. p. I Z2. Heron gris Briss. Ornith. V. p. 4 I 2. pl. XVI. 1. Ardeat grifea, Linn. Genl. 84. 2,2.

3!6. Ramicr (Pigeon) Burr. IV. p. 36r. pl. XV. BRrss. Ornith. I. p. 89. Coluniba Palumbus, Linn. Gen. 104. I 9.

397. Brunet (Merle) du Cap. Buff. VI. p. 88. Varieré di Merle brun du Cap. de M. Briss. Ornith. II. p. 259. Turdus Capenfis Ë Linn. Geil. 107.17.

318. Gobemouche rouge et vert. Bufr. XII. p. 209. Briss, Ornith. III. o. Muficicapa bicolor mihi Linn. Gen 63 . o.

319. 1. L. Bouveron Buff. VIII. p. 120. Pyrrhula africana minor BRTsS. Ornith. III. p. 319. pl. XVII. I. Loxia fusca Linn. Gen 109. 46 ,

$\mathrm{E} 2$

2. 
Pl.

-2. Bec rond a ventre roux, Buff. Vill. p. I23. Briss. Orinith. Ill: ob Loxia fufciventer LinN. 109. 0.

320. Goërtan (Pic) Burf. XIï. p. 36. Pizus Briss. Ominth. III. o. Lins.

Gen. 5?. o. Crimton rumped wood pecker, Latr. Birds 1. p. $5^{8} 3$.

32 I. I. Guimegat. Burf. VIII. p. 8r. varicté du Pinfon Brtss. Ormith. Ilt.

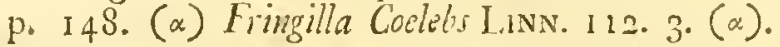

2. Le Mordoré Burf. Vill. p. 88. Briss. Ornith. o. Enberiza fusco fullia mihi Linn. i Io. o.

S22. Jaçana Buff. XV. p. 2SS. pl. XVI. le Chirurgion brun, Briss. Ornith. IV'. p. I 5 . pl. XI. f. I. Parra Facma, Lins. 92. 3.

323. Blongios (Hcron) Burr. XIV. p. I27. Aricola Briss. Ornith. V. p. 497. Ardea mimita, Linn. Gen. 84. 26.

324. I. Manakin a gorge blanche, Burt. Vili. p. I65. Bruss. Ornith. IV. p. 441. pl. XXXVI. fig. I. Pipra gutturalis, LinN. Gen. II510.

2. Nanakin varié, Buff. Tlll. p. 167. Nanakin a front blanc Briss. Onith. IV. p. 457. pl. XXXVI. 2. P'ipra ferena, Linn. I I5. I I.

325. Grand Jingoulevent de Cayene, Burr. XII. p. 255. Caprimulgus Brafrikenfis navius (a) Bruss. Omitho. II. p. 485. Caprimulgus Linv. Gen. II 8 . 2. $(\alpha)$.

326. Rollier de Sencgal, Burr. V.p. 177. 2. Briss. Ornitlo. o. Coracias, Linn. Gen. 51. o. fwallow tailed Indian Rollar Edw. glean 327. Sensgal Roller LATi. birds I. p. 408.

327. Corneille de Senegal, Buff. V. p. 22. Briss. Ornith: I1. p. 19. ( 2 ).

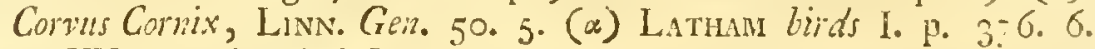
pl. XV. white brealted Crow.

328. Casfique verd. Burf. V. p. 331 : Censficus viridis Briss. Omith. Il. O. Oriolus viridis, Linn. Gen. 52. o. Ciefted oriole Latilial birds I. ?. 422. 11. 3. B.

329. Tourrocco Buff. IV. p. 395. Briss. Omith. I. o. Colmmba (Turetr) LINN. IO4. 0 .

330. L.e beau Tamatia, Bufr. XIII. p. I †. Bucco mayanen/is Briss. Omith. IV. p. I o2. pl. VII. fig. 3. Bucio pitus mihi Linv. Gin. 56́ o. benutiful Barbet Latham p. $498 .+$.

33. Barbu a gorge Jaune, Burf. XIII. P. 152. Bucco Philippenjis Briss. Ornith. IV. p. 99. pl. Vil. 2. Butco flavigula, Lin w. Gen. 56. o. yellow Throated Barbet, Lathan I. 50. 0. $7^{\circ}$

332. Rufalbin (Coucou) Burr. XII. p. 23. Coucou de Senegal Briss. OFnith. IV. p. 120. pl. VIII. I. Cuculus Jenegallenfis, Linn. Gen. $57 \cdot 6$.

333. Epouvantail ou Guiffette noire, Burf. XIV. p. 90. Sterna nigra Briss. Urmith. VI. p. 2II. pl. XX. I. Sterna fisfipes, Linn. Gen. 77. $7 \cdot$

334. Marcinpecheur Crabier, Burf. XIll. p. 269. Ispida Cancrophaga, Briss. Ornith. o. Alcedo Cancrophagus, Linn. Gen. 62. o. Crabeating kingsfisher, LaTH. birds I. p. 618.9.

335. Varieté de l'Amazone a tete blanche, Burf. XI. p. 299. Pfittasus Martinicanus gutture rubro, Briss. Ornith. IV. p. 244. Pfittacus léucocephalus, Linn. Gein. 45. 3o. Whire headed Parrot Lath. Birds, I. p. 279.88. 
Pl.

336. Papcgay de Paradis, Burf. XI. P. 33๘. Pfittacus luteus infule Cula, briss. Ornith. IV. p. 308. Hattacus Patudifi, Linn. Gen. 45. 3\%. Pandife Parrot Latn. budj I. p. 300. n. 105.

33\%. Hoazin (Faifan!. Butr. IV. p. 146. Plaifiames Briss. Ornith. I. o. P'hafianus, Linv. Gén.101. o.

33S. Marail Burf. IV. p. I53. varieté de J'Ýacon?

339. Merle folitaire des P'ilippines, Burr. V1. p. 50. Briss. Omith. II. p. 2?2. Turdus Philippenfis, Lins. o.

340. Tourne pierre, Buff. XV. p. 205. Coulon chaud (Arenaria) Briss. Ornith. V.p. 132. Tring anterpres, Linn. Gen 87.4 .

34 I. I. Verd brunce Buff. VII. p. 255. le verdier des Indes, (Chloris) BRIss. Ornith. IlI. p. 195 Fringilla butyracea, LinN. II 2 =. 2. Verderin Burf. I'II. p. 253. Briss. Ominth. o. Linn. Gen. il 2 o.

342. Canard Cravant, Buff. . . . Cravant Briss. Ormith. VI. p. 305. Amas Bernida ( $\beta$ ) Linv. 67. 13 .

343. Le Commandeur, Buff. V. p. 293 . Troupiale a ailes rouges, Briss. Ornith. II. P. 97. (a) Oriolus Phoenicous, Lisn. Gen. 52. 5. (a) voyez pl. 402 . er 236 . 2. et $53^{6}$. Redwinged Starling, Caterb. Carol I. pl. I 3 . Redwinged oriole Latr. lira's $423.13^{\circ}$

344. Castique hupé Butr. V. p. 332. Casficus criftatus Rriss. Ornithoo. Oriolus criftatus, Linv. Gen. 52. o. crefted oriole Lath. birds I. F. 421.3.

3+5. 1. Epeiche de Canada, Bufr. XIII. p. sor. Picus varius Camadenils, Brıss. Orintlı. IV. p. 45. pl. Il. f. 2. Picus leucomelas mihi Linn. Gen. 59. o. Canadian fpotted woodpecker, Latu. Burds I. p. 569.

2. Perit Pic rayé de Senecral, Bufr. XIII. p. 37. Petit Pic de St. Domingo, Bkiss. Ormth. IV. p. 75. pl. IV. fig. 2. Picus pasferinus, LinN. 59. 6. Gold backed woodpecker, LaT11. birds I. p. 586. 33.

346. Oye de C:mada, Burf..... Briss. Omith. VI. p. 2?2. pl. XXXVI. Anals Canadenfis Livv. 6-. I 4.

347. Oye Buff. .... Anser gaimienfis, Briss. VI. p. 265. Anfer Cygneus Linv. Gen. 67. 2.

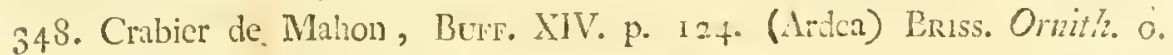
LiNN. O.

349. Crabier bleu a col brum, Burf. XIV. p. 133. Briss. Oinith. V.p. 48 4. Ardea coerulea, Linn. 83. 17.

350. Demie Aigrette, Bufr. XIV. p. 103. Ardea Briss. Oimith. V. o. Aideal lewiogafter, Linn. Gen. 84.o.

351. I. Rosignol de Murnille, Burf. IX. p. 247. Ruticillı Bufr. Briss. Ornith. III. p. 403. Motacilla Plocmicurus, Linn. 114.34. 2. La Femelle.

352. Grande Poule d eau, Buff. XV. p. 202. Briss. Orniti. V. o. Fulica major, Lisn. Gen. 91. o.

353. Avocette Buff. XVI p. 279. Briss. Ornith. VI. p. 538. Avocetta recursirofira, Linn. Gen. 89. I.

354. Podobé (Merle), Buff. VI. p. 55. Meiula Briss. Ornith. III. o. LiNN. O.

355. l'Azurin (grive), Bufr. VI. p. 118. Briss. Otnith. III. o. Turdus Cyanurus, LiNN. 107. 0. 
356. Niutin pecheur a tête bleue, Burf. XIII. p. 285 . Ispida niggra, Briss. Ornith. o. Aliedo nigra mili Linv. Gen. 62. o. Ulue headed Kingstisher, Latnhr birds $\sigma_{31}, 20$.

35.' Bec en Cifean, Búfr. XVI. p. 262. pl. 1.2. Rhyncopfalia dorfo nigto, Briss. Omith. VI. p. 223. pl. XXI. 2. Rhyncops LinN. Gen. 78. I.

558. Oranvert (Merle) Burf. VI. p. 68. Briss. Ormith. II. o. Turdus Erythrogafter nibi Lisn. Gen. 107. 0.

$35 \%$ Pcrruche a alies varicés, Bufr. XI. p. 362. Pfittaca Cayanenfis, Briss. Ormith. IV. p. 334. Linn. Gen. 45. o. yellow wnged Parrakect, Late. lirds T. p. $2+4 \cdot 4.2$.

360. Crik (Perroquec) a face bleue, Buff. XI. p. 320. Pfitacus Amuzoni-

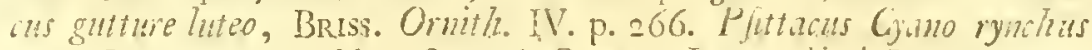
mihi Linn. 45. 0. blue fronted Parrot, Latu. birds I. p. 291. n. 95.

361. 1. Rouge gorge, Buff. IX. p. 285. pl. XI. Rukecula Briss. Ornith. III. p. 418. Motacilla rubecula, Linn. Gen. I I 40" 45 .

2. Gorge bleuc Burf. IX. p. 300. Cyanella Briss. III. p. 4 I6. Motacilla Surcira, Linn. II 4.37 .

302. V'nncou armè du Senegal, Bufr. YV. p. 98. Briss. Grnith. III. p. III. pl. X. 2. Parra Senegalenfis, Linn. 92. 2.

363. I. Aloucte Brff. IX. p. I. Briss. Onith. III. p. 335. Alanda Aivenfis Linn. Gell. 105.1.

^. La Calandre, Buff. IX. p. 7o. Brrss. Omith. III. p. 352. pl. XX. 2. Alauda Calandra, Linn. Gen. 105.9.

364. 1. Serin de Nornmbique, Lurf. VII. p. 72. Bruss. Ornith. III. o. Fringilla Lepida, LunN. Gen. I I2. I4.

2. La Femellc.

365. Maubeche tachctée, Burf. XIV. p. 325. Catidiis naeria BrIss. Oinith. V. p. 230. Linn. Gen. 87. o.

366. Maubeche grife Buff. XIV. p. 3=6. Culidris grifea Basss. Craith. V. p. 233 . Tringal grifea, Linn. 87. 16. R.

jor. Le Pungoin Burf. . . . . Allat major Briss. Omith. VI. p. S 5 . pl. VII. Aliu impermis, Linv. 69. 3 .

368. Le Kiolo (Rale) Burf. XV. p. 253 . Brisson Ornith. VI. o. Ratlus Gayyzinenfis mihi Lins. o. le même que cclui de la planche 753 .

369. Le petit prille en queue, BuFF. XVI. p. II 3 . Leptumes candidus, Briss. Omith. VI. p. 485 . pl. XLII. 2. Phileton wethereus, Liny. Gen. 74. 1.

370. Houtou ou Momot, Burf. XII. p. 103. Momot Bruss. Omith. IV. p. 465 . pl. XXXV. 3. Ramphafios momota Lind. Gen. 46. 6. Biratilian Momot, Latham birds I. p. 338. 1. pl. Iо. Brafilian fawbilled Roller Lin. 348 .

371. Le Piverd. Burf. XIII. p. 9. pl. 1. Briss. Ornith. IV. p. 9. Picus ziridis, LlnN. Gen. 59. I 9. green woodpecker Latium birds I. p. $5 ? \div \cdot 25$

3.2. 1 Grimpercau de Muraille, Burf, X. p. 233. pl. VII, Bass. Ornith. II. p. -6. Certhia muraria, Lins. Gen. 65. 2. Wald Creeper LaTH. lirds I. p. 730. D. 32.

2. Ia Fenelle. 
$\mathrm{Pl}$.

37 3. La blanche Coiffe (geay), Burf. V. p. r6:. Gedy de Cayenne Briss. Ornith. IT. p. 52. pl. IV. 1. Cermus Cayanenfis, LiNs. Cen. 50. 9. Cayenne Jay LATH. birds 1. p. $=88$. n. 22.

374. Tcha-Chert-bé (Piegriefche), Buff. 1r. p. 95. Briss. Oimith. II. o. L-INn. Gen. 44. o. White headed flirike, LATH. birds I. p. iSo. 32.

375. Capmore (Troupiale) Buff. V. p 3 II. Briss. Omith. II. o. Oitolus LinN. o. le male jeune, Weever oriole Liath. birds I. p. 435. n. 21.

3:6. Le méme adulte.

3--. Becarde (Piegriefche) Burf. II. p. 93. fenclle de celui de la planche, 304. fpotted Cayenne fhrike Latian birds 1. p 189.

378. Ouete (Cotinga), Burf. VIII. p. 208 . Cotinga rouge Briss. Ornith. 11. p. 35 I. Ampelis Caronfex, Lins. Gen. 108. 3.

359. Oye de Egypte Burf. . . . Briss. Omith. VI. p. 2S4. pl. XXVII. Anas Egyptzaca, Linn. Gen. 67. 10.

380. Queuc en Eventail (Grosbec), Burf. MT. p. r9i. Briss. Ornith. o. Loxilt flubellum mihi Linn. Gen. 1 1 0.0.

$3^{S 1}$. Le Piahau, (Gobemouche) Burf. VIII. p. 416. grand Gobemouche noir de Cayenne, Briss. Ornith. II. p. 386. Mafcicapa cruenta mihi Linn. Genl. II 3 . O.

392. Pinguin Burfon. . . . Cataractes, Bruss. Ornith. VI. p. 102. Phä̈ton demerfus, Lins 74.8 .

-383. Ama verd. Butr. XI. p. 27 I. Briss. Omith. IV. p. 199. Pfittacus feverur, Linv. 45. 6. Brafilian green Maccaw. LaTH. Birds I. p. 20 S. 11. I9.

394 . Papegay a tete et gorge bleue, BuFf. XI. p. 34i. Perroquet a tete bleue de la Gunjane, Briss. Omith. IV. p. 247. Pfitalus Cyanogul.s mihi Linn. Gen. 45. o. Blue headed Parror Edw. pl. 314 . Lati. birds I. p. 301. n. 107 .

385. Le grand Duc (Hlibou), Burf. II. p. iz r. pl. VIII. grand Duc de virginie Beiss. Oinith. I. p. 48 4. Strix Bulo, Linn. Gen 43. I. great homed Owl from virginia EDw. birds II. Tab. 6o. Virginian Eared owl. LatI. birds I. p. 219.2 .

386. 1. Therefe jaune, Burr. VIIt. p. 83. Briss. Oinith. IU. c. Emberiw mexicana mihi Linv. Geli. 110.0.

2. Bonjour Commandeur, Berf. VIII. p. 93. Coccothrauftes Briss. Oimith. III. o. Emberiza pileuta mili LinN. 110. o.

387. Le kuthgekef, Burf. XVI. p. 281 . Gavia cinerea naeria, Briss. Ornith VI.p. 185. Latrus canus, Linn. Gerl. 76. 3. ( $\alpha)$.

388. s. l'Agripenne ou Ortolan du Riz, Burf. Ví.. p. 49. Ortolan de la Caroline, Briss. Ornith. III. p. 282. Emberiza Agrepenisis, LinNo Gथा\%. 110. I6.

2. Varieté du même, de la Louifanie.

3. Ortolan.

Je nel trouve point cité dans l'ouvrage de M. Buffon.

389. l'ibis Buff. XV. p. 21. pl. x. Briss. Ornith. V. p. 349. Tantalus Ibis Linn. Gell. $85 \cdot 3$.

390. Coucou du Cap de bonne Esperance, Briss. Ornith. III. o. Je ne le trouve point chez M. Buffon Cape Cuckow Latir. birds p. $5 \sqrt{3} \cdot 2$. 
P1.

\section{$24)$}

391. I. Fignier huppé, Burf. IX. p. 461. Ficcdula Briss. Ornith. IJI. o. Motacillı Galeata, mihi LınN. Grn. i I 4. o.

2. Fignicr noir Buff. IX. p. 46 a. Ficedula nigra Briss. Ornith, III. o. Motacilla nigra, Linn. 11 f. 0 .

392. Le Casque noir Burf. VI. p. 85. Merle a tête noire du Cap de bonne Esp. Briss. Omith. Suppl. p. 47. pl. III. fig. 2. Turdus merula atricupilla, Linn. 107.418.

393. I. Le gris albin Buff. VI. p. I97. pl. XIV. Briss. Ornith. Juppl. p. 47 .

2. I'Orchef Burf. VI. p. 196. Briss. Ornith. III. o. Loxia regiza mihi LiNN. I I O. O.

3. La Nonnette, Burf. VI. p. 196. Briss. Ornith. III. o. Loxit cat- + cullata, mihi Linn. i Io. o.

394. Tourterelle Commune, Buff. IV. p. 390. Tourterelle a Collicr Briss. Oruith. 1. p. 95. Columba riforia, Linn. Gen. 104. 33 .

395. Tamatia a Collier (Barbu), Burf. XIII. p. 1 45. Bucco Briss. Omith. IV. p. 92. pl. VI. f. 2. Bucco Cispenlis, LinN. Gen. 56. I. Collared Barbct Latir. Birds. I. p. 497. 3.

396. 1. Le Rouge gorge bleu, 13urr. X. p. 308. Rulecula Carolinenfes coerulea, Briss. Ormití. IIl. p. 423. Motacilla fualis, Lann. Gen. $114.3^{8}$.

2. I a Fomelle.

397. Varicté de l'Ecorcheur voyez planche, 30. 2. Lomfian. fchrike Latul. lirds I. p. I62.

393. I. Grive de la Gunjane, Burr. V. p. 399. 1. Turdus Briss. Ornizi. II. p. 205. Turdus lliacus P. Linn. 10\%. 3.

2. La Grivelette de St. Domingo, Buff. V. p. 4t1. ficedula Canderfis cinerea, Briss. Ormith. III. o. p. 524. pl. XXVIII. 1. motach la Canadenfis, Linn. Gen. $114 .{ }^{2} 77$. EDw. glan 252. tig, intig.

399. Cicogne noire Buff. XIII. p. 396. Cicogne brune Bruss. Ormith. V. p. 362. Ardea Citoma nigra, Linn. Gen. 8+. \%.

400. Grebe Cormue, Burf. XV.p. 365 . pl. 19. Colynluts cormatus, BrIss. Ormith. VI. p. 45. Colymbus crifiatus LiNN. Gen.75.8.

401. Cresferelle Buff. II. p. 48. p]. IV. Tinnunculus Briss. Omith. I. p. 393. Falco Timunculus, Linn. Gen. 42. i6. Keftril falcon Lathin Birds 1. p. 94. n. 78 .

402, Le Commandeur, Burf. V. p. 293. Troupiale a Ailes rouges, Briss. Ornith. II. p. 97. Oriolus Phaeniceus, Linn. Gen. 52. 5. Redwenged oriole, LATH. birds I. p. 428. I 3 .

403. Canard farcelle de Cayenne, Burf. . . . . Canard Sarcille de Virginie Briss. Omith. VI. p. 455. Linn. Gent. 67. o.

40+. 1. Grand Grebe, Burf. XV. p. 375. Briss. Ornith. VI. o. Colymbus major mihi Linn. Gen.75. o.

2. Petit Grebe cornu, Buff. XV. p. $3^{68 . ~ B r i s s . ~ O m i t h . ~ V I . ~ p . ~} 50$. Colymbus Auritus, Linn. Gen.75. 8.

405. La Spatule, Burf. XIV. p. 202. pl. X. Platea Briss. Ornith. V. p. 352. Platalea Leucorodia, Lins. Bo. I.

$51+$ 406. Grive Buff. V. p. 396. Turdus lliacus, Briss. Ornith. II. p. $=08$. H. XX, I. LiNN. Gin. 107. 3. 
T1.

40\%. Permche Pavouane Jeune, royez pl. $16 \%$.

400̈. Papegay violet, Bufr. XI. p. 3+3. Pfittacus Violaceus mihi Briss. Ornit/2. IV. O. Linv. Gen. 45 . O. little dusky Partot Eew. birds pl. 3!5. Latil. birds I. p. 302. 11. 108.

409. Aigle Commun, Buff. I p. 120. Aquila valeria, Briss. Ornith. I. p. 434. Fillco melanuetos, Linv. Gcn. 42. 2. Black Eagle Latir, birds. I. p. 28 .

410. Le grand Aigle, Bufr. I. p. 107. Aquila chryfaetos, Briss. Ornith. I. p. 43 I. Faico Chryfaetos, Linn. Gell. 42. 5. golden Eagle Lath. I. p. $3^{\mathrm{I}}$.

411. Pygargn:e, Bufr. I. p. I.38. Aigle a tere blanche, Briss. Ornith. I. p. 422. Falco Leucocephalus, Linn. Geir. 42. 3. White tailed Eagle Latham Birds I. p. 32. cinercous Eagle ibid p. 33 .

4 I2. Epervier Burf. I. p. 3 I S. pl. XI. Accipiter Briss. Omith. I. p. 3 Io. Falco Nifus, Linn. 42. 31. fparrow hawk. Lathim birds I. p. 99. n. 85 .

4I3. Jean le Blanc, Butr. I. p. 1?4. Briss. Ornith. I. p. 443 . Linn. 42. o. Lathan birds 1. p. 39.

414. Le Balbufard, Burf. I. p. 144. Aigle de Mer, Briss. Ornith. I. p. 440. pl. XXXIV. Falco Halinetos, Linn. Gen. 42. 26. osfrey Eagle LATII. I. p. 45 .

4I5. l'Orfaye (Aigle) voyez pl. in

416. Aigle de Pondichery, Buff. I. p. 490. Briss. Ornit/r. p. 450. pl. XXXV. Falco Indus milhi Linn. Gen. 42. o. Latis bird's I. p. 41.

417. Petit Aigle d'Ameriqque, Burf. I. p. 198. Briss. Ornith. I. o. Falco americanus miki Linn. Gen. 42. o. Red Throated Falcon, Lath. birds I. p. 97. 82.

418. l'Aurour Buff. I. p. 326. Aftur. Briss. Onith. I. p. 317. Falco palumbarills, Linn. Giell. $42.3^{\circ}$. Goshawk Lath. birds I. p. 58.vid. pl. 46 I.

419. La Bufe Buff. I. p. 290. Briss. Ornith. I. p. 416. Falco Buteo, Linv. Gun. 42. 45. Common buzaard, LАти. Bird's I. p. 48.

420. La Bondrée Burf. I. p. 293 . Briss. I. Ornith. I. p. 4 IO. Falco apizo. rus, Linn. Gen. 42. 28. Honcy Buzzard, Lath. Birds I. 52.

421. Faucon hagard Burr. II. p. 9. pl. II. Falco gentiles, Briss. Ornith. I p. 339. Linn. Gen. 42. I3. hagard Falcon Latil. birds I. p. 66. 49. B.

422. Mlan Royal, Burf. I. p. 280. pl. VII. Briss. Ornith. I. p. 414. pl. XXXlli. 2. Falco Milvis, Linn. Gell. 42. I2. Lite Lath. Birds. I. p. 61, n. 43 .

423. Autour blanc Bufr. I. p. 33 I. Gros Bufard Briss. Ornithe I. p. 398. Fulco albescens milhi Linn. Gen. 4,2. 0.

424. Le Buzard Buff. I. p. 308 . pl. X. Bufard des matais Briss. I. p. 407. I. Falio neruginofus, Livs. Gen. 42. 29. Moor buzard Lath. birds I. p. 53. n. 34 .

425. Grand Vautour. Burf. I. p. 22 I. Vhltur cinereus Briss. Ornith. I. p. 453. Vultur Linn. Gen. 42. o. Cinereous Vulture Lath. fyn. I. p. I 4. Black vulture, Lath. I. p. 16.

426. Percnopiere Bufr. I. p. 209. Vultur Percnopterus, Briss. Ornith. I. p. 464. Lists. Gen. 42. 7. Alpine Vulcure, Lath. Jynopf. I. p. I2.

427. Vautour brun, Buff. I. p. 234. Briss. Ornitl. I. o. Vultur fufous mihi Lanv. Gen. 42. o. Malthefe Vulture, Lath. Jyn. I. p. I5. 
pi.

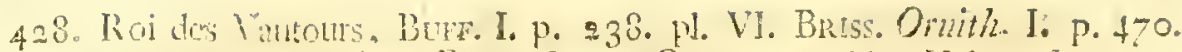
pl. XXYT\}. V́ulur Papa, Linn. Gen. 41. 3. king Vulture Lathum Jy. I. p. $\%$

429. Petic Vutom Berf. I. p. =30: Vantour a tete blanche, Briss. Omith. 1. P. 466. Linn. Giar. 4i. O.

430. Tiercelet de Faucon le mêtne que celui de la planche 42I. mais de trois ans.

43. S Hobrean Butron II. p. 43. pl. IL. Briss. Omaith. I. p. 375 .

432. \{lutbeteo $\beta$ Linn. Gein. 42. 14. Latman birds I. p. 103. ถ.

433. Le Pinen Braf. IV. p. I. Tab. I. Phalianus Paro, Briss. Ornith. I. p. 28 1. pl. XXViI: Pavo crititatus, Linn. Ger. 98. 3.

434. La Fenceric.

435. Le Duc (1tibou), Burr. IT. p. Iา1. pl. VIIT. Bubo Briss. Ornith. I. p. 47. Jim Brobo, Linv. Ger. 43. 1. grent eared Owl. Latis. bids I. p. $116 . \mathrm{I}$.

436. Le petit Duc, Butf. II. p. r2т. pl. X. Briss. Ornith. I. p. 495. firis foops Linn. Gen. 43. 5. fcops eared Owl. Lath. birds I. p. 129. 15.

437. Le Chat huant Burf. H. p. $6_{3}$. pl. XI. Briss. Orvith. I. p. 500. Strix firidula, Linn. Gen. 43. 9. Tawny owl. Latr. tirds 1. p. 139.27.

438. La Chouette, Burf. II. p. 176. pl. XIII. Briss. Ornith. I. p. 511. frix Ulula Linv. Gen. 43. 10. Brown owl. Lath. 140. 11. 28.

439. La cheveche Búrf. II. p. 183. pl. XIV. Nostua minor Briss. Ornith. I. p. 514. Jirix pusferina, Liñn. Gen. 43. I2. little Owl. Latri. I. p. 150 .

440. La Ficfayc, Burf, II. p. r68. pl. XII. Petit chat hume Briss. Omitt. I. p. 503. Jitrix flammea, Linn. Genl. 43. 8. White owl. Lath. birds I. p. I $98 . n .26$.

44 1. La Hulotte, Bupr. II. p. 158. Briss. Omith. I. p. 50\% ftrix Aluco, Linn. Gell. 43. 7. Aluco owl. Latri. birds J. p. I 34. 11. 20.

442. Chat huant de Caycnne; Burr. II. p. 202. Briss. Ormith. I. O. Lisn. Ge'l. 43. o. Cayenue owl. Latio birds I. p. 146. 11. 35.

443. Solibufe Burf. T. p. 303. pl. IX. Faucon a Collier, Briss. Ornith. h. p. 345. Fulco Wubbuteo, Linn. Geno 4‥ 14. Ringtail Lath. birds I. p. 89. 1. 75 . vayez la femelle planche 480 .

4.4. Emerillon de Cajenne, Bufr. II. p. 64. Briss. Omith. I. o. Lins. Gen. 42. O.

445. Piegriefche mife, Butr. II. p. 70.pl. VI. Lamius Briss. Ornith. o. Lanius Collwio Lins. 44. 12. great cincreous fhrike, Latil. birds I. p. 160 .

446. Gerfaut Bufron I. p. 339. pl. XIII. Briss. Ormilz. I. p. 370. 373 . pl. XXXi. Falio Gyrfaico, Lins. 42. 7. voyez ausfi pl. 210. 452. white Jerfalcon, Lath. birds I. p. 83. n. 69.

447. Le Rochier Bupf. II. p. 56. (varicté de la Soubufe) Faucon de Roche Briss. Ormath. I. p. 349. Sulbuteo 'ß. Lins. 42. 14. Stone filcon Laril. birds I. p. 93. 11. 77 .

44.8. I. Troupiale tacheté Burf. V. p. 306. Carougo Briss. Ornith II. o. Oriolus melancholimi, Livn. Gen. 52. 17. Schombirger EDw. Livds I. pl. 85. Latru. birdo. I. p..441. 11. 31. 2. Lat Femelle. 
).

479. Picte male Bufr. XV. p. 427. Merganfer albellus Briss. Omith. V\%. p. $243 \cdot$ pl. XXIV. J. LIN . Gen. 6.8. 5.

450. La Finclle.

4.51. Le Kamichy, Burr. XIV.p. 40. p?. I. Aahima Briss. Omith. V. p. 518. Paldinedea comuta, Lins. 81.!.

452. Courrancoul a ventre rouge, Befron XI. p. 400. Troon Brafilinfis siridis, briss. IV.p. 173. Trogon Cawnal, Lins. Geit. 55. ュ. red bellieil Curucui, L Lath. Birds I. p. 485 . I.

45. 1. Gobemouche roux de Cayeime, Burf. VIII. p. 353. Briss. Omith.

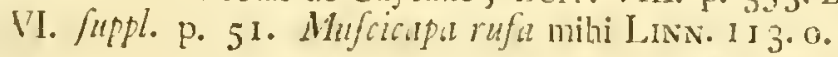

2. Caudec (Gobemonche) Berfon. ITI. p. 406. Mufcicapa Bniss.

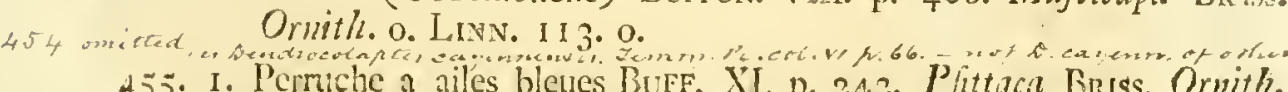

455. I. Perniche a ailes bleues BuFF. XI. p. 242. Plittacal Briss. Ornith. IV. o. P'littacus Cyanopterus, Linv. 45. o. Bluewinged Parrakec, Lisir. livids I. P. 317 . 11. 124.

2. MArmanon Perruche, Bufr. XI. p. 245. Briss. Ornith. IV. o. Linn. Ger. 45. o. Otaheicun blue Parrakect Latito birds I. p. $=55 \cdot 59$.

456. I. Toui-eté a tece d'or Burf. XI. p. 395. Pfittacula Brafilienfis Urofygio cyaneo, Briss. Ornith. IV. p. $3+3$. Pfittacus pasferinus, Lind. Gen. 45. 47. Goldheaded Parrakect Latir. I. p. 321. n. 331 .

2. Sofàvé (Toui) Burf. XI. p. 39r. Brtss. Omith. IV. o. Linn. Gen. 45. o. Yhttaills notutus, mihi. Cayenne Parrakect Lath. birds I. p. 320. ก. I 3ิ०.

457. Mutruche Bufron II. p. =12. pl. XV. Briss. Omith. V. p. 3. pl. I. fig. 1. Struthio Camelus, Linv. Gen. 96. I.

458. Harfarg (ilibou) Buff. II. p. 197. Aluco albus Briss. Ornith. I. p. 522.Jirix Nycteli Linn. Gen. 43. 6. fnowy owl.Latu. birds I. p. 132.11. 17.

459. Oifenu faint Martin Buff. I. p. 365 . Briss. Ornith. I. p. 404. Follco Cyanelts Linn. Gein. 42. 10.

460. La Harpaye Befr. I. p. 316. Buteo mifus Briss. Ornith. I. p. 404. F́lco Harpyju Linn. Gen. 42. o. Harpy Latio birds I. p. 5 I.

461. Autour Bufr. I. p. 326. Briss. Omitl. I. p. 317. Fulco Palumbarius Linn. Gen. 42. 3\%. Goshawk. Latil. birds 1. p. 58. n. 39.

463. Gerfaut Buff. I. p. 539. pl. XII\%. varicté de 210 . et 446 .

463 . Chonetre a longue queue de Siberie, Briss. Ornith. I. o. Linv. Gen. 43. 0. Ural owl. Latrian tirds I. p., 14 4.8. 17. 3\%.

464. Epervier de Caycinne, Bufr. I. p. 336. Briss. Ornith. I. o. Linn. Genz. 42. 0. great billed Falcon Lath. birds I. p. 103. n. 89.

465. Encrillon de St. Doningo, Burf. II. p. 64. le méme que celui de la planche 444. litele Falcon LuTit. lirds I. p, 110. 11. 94.

466. Epervier Bufr. I. p. 318 . Briss. Omitle. I. p. 310 . Falco Nifus Linn. Gen. 42. 31. Lath. birds I. p. 99. n. 85. le même que 4 I2.

467. Pigeon Commun, Burf. IV. p. 306. Pigeon Domeftique Briss. Ormith. I. p. 68. Columb domeltica, Linn. Cent. 104. I. 3.

468. Emerillon Burf. II. p. 59. pl. V. BRiss. Ornith. I. p. 38s. n. 9.3. Fal. co Aefalon, Linn. Gen. 44. o. Falconers Merlin, Latu. I. p. Iog.

469 . Faucon noir Buff. II. p. 22. varicté du Faucon. Black Falcon Latr. birds I. p. 60. n. 49 .

$\mathrm{G} 2 \quad \therefore \quad 47^{\circ}$ 
ịl.

470. Fatuen forts Burr. II. p. \%. pl. 1. Briss. Oinith. I. p. 339. Falea Gentilis, Linn. Cen. 42. fig. I 3. yearling Falcon, LaThai birds I. p. 65 .

47. Femelle de la Cresferelle voyez le mâle 401.

4-2. Mulan noir Buff. I. p. 286. Briss. Ornith. I. p. 4 I. n. 34. hlack Kite L.AT1. birds I. p. 62. n. 63. Falco migrims, mibi Linn. Gen. 42. 0 .

473. Petit autour de Cayenne, Buff. II. p. 337.2. Cayenne Falcon Lath. birds I. P. 59 .

474. La Gelinotte Berf. 11I. p: 336. Briss. Ormith. 1. p. 191. Tetrao Bomilia, Linn. 103.9.

475. La Funclle.

- 476. I. Ecorcheur des Philippines, Bitr. II. varieté de l'Ecorcheur.

๑. Piegricfiche grrife de la Lonifimic varieté de la Piegriefche grife de la planche $4+5$.

47-. I. Piegriefche du Cap de boune Esperance, Bufr. II. p. 75. autre varicté de la Piegriefche grife pl. 445. Collired Chrike Latham burds l. p. 163.7 .

: 2. Piegricfche rousfe du Senegal, Buff. II. p. 83 . le même que la Piegriefche rousfe d'Europe, on l'ecorcheur BRiss. Orvith. II. p. I 47. variety of wood Chat fhrike Lathaм birds I.p. I 7 o.

478. Tanas (Faucon) Büf. I. p. 41. Briss. Ormitl. I. o. Fulco Piscator, Linn. Gen. 42. o. Fifching Falcon Lath. birds I. p. 95. n. 80.

4.9. Piegricfche du Scnegal, Burf. II. p. 84. varieté de 4:7. 2.

480 . Soubufe (mâle) voyez lia femelle pl. $44 \hat{\jmath}$.

481. Le Geay Refr. V. p. I 46 . pl. 8. Briss. Ornith. II. p. 4\%. Corvus Elandarius, Linn. Gen. 50. 7. Jay Lath. birds I. p. 384. ig.

482. Le Jupuba, Burf. V. p. 328. Castique ronge de Brafil Briss. Ornith. II. p. 93 . Oridus Plutmorihous, LINN. 52. 5. B. Red rumped oriole
LATH. bird' I. 1. 4.20. 2.

483. Corbine Burf. V. p. 6I. Corneille Briss. Grmith. II. p. 12. Corys Coroné, Liviv. Geiz. 50. 3. Carrion Crow. LATH. 370. 2.

484. Le Freux Bure. V. p. 75. Briss. Ornith. II. p. I6. Corme frugilleges Lann. Gen. 50. 4. Rook Lath. birds I. p. 372. 4.

485. 1. La Linotte, Burf. VII. p. 83. pl. 1. Linuria rubra Bruss. Ornith. III. p. 135 . Fringilla Camabind, LinN. I I 2.28.

5 486. 2. Le Cabaret, Burr. VHI. p. 109. Linaria minimu Briss. Omitits. III. P. 1.f. Fringilla minima mihi Linv. 112.0. 3. Le Tarin Bufr. VII. p. 309. Ligurimus Briss. Ornith. III. p. 65.
Fringillie fpimis, Lisn. Gen. I 12.25.

436. Roliier de l'Europe, Burr. V. p. 184. Garrulus Aigoratenfis, Briss. Omit\%. II. p. 64. Coracias Gumrulus, LinN. Gen.51. 1. Garrulous Roller, LATH. birds I. p. 406. I. 48\%. Tourterelle de St. Domingo, je ne le trouve pes dans l'Ourrage de
M. Burron.

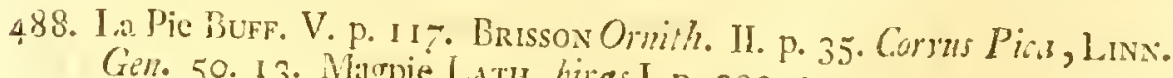
Gen. 50. 13. Magpie LATi. biras I. p. 392. 29.

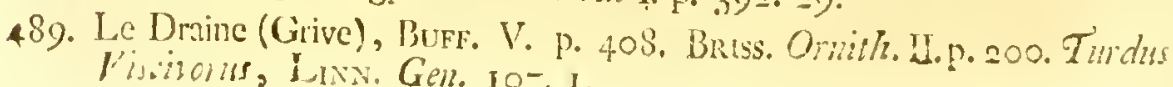


420. La Litome Bufr. V. p. 417. Grive moyenne a pieds noirs Brišs. Oinith. II. p. 21 1. Turdus pilitrs, Lins. Gen. 101. 2.

491. Migcon de Nicobar, Buff. IV. p. 37. Briss. Omith. I. p. 153. Columbía Nicobarica, Linn. 10 \&. 27

492. I'Eperronier (Pan) Bufr. IV. p. 12 1. Phaifun Pion de la Chine, Briss.

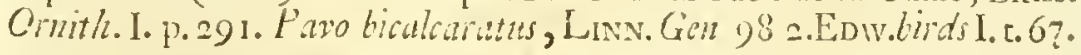

493. Li Femelle.

494. La Lagopede Buff. III. p. $33_{3}$. pl. IX. Triss. Omili. 1. p. a I6. Tetrao Lagopus Linn. Gen. 103 . 4.

495. Corbean Burr. V. p. I6. pl. II. Briss. Onith. II. p. 8. Corvus Corax Linn. Geil. 50. 2. Raven. Latit. birds I. p. 36\%. I.

496. Le Manucode, Buff. V. p. 223. p!. XIII. Munucodiata minor Briss. Ornith. II. p. 136. pl. XIIT. 2. L'ardifea regla, Livn. Geil. 54. =. EDw. Birds. II. I I I. king bird LATH. I. p. 475 .

$49 \%$ 1. Ortolan de Neige, Burf. Vili. p. 38. Briss. Ornith III. p. 285 . Enberiza Nivalis, Linn. Gen. I10. 1. Evw. Birds p!. 12б.

2. Crolan des rofeaux, Buff. VIll. 16. Hortrlanus Arndinaceus, Briss. Ornith. III. p. 274. Linn. Gen. I Iо. o.

498. Kakntoes a huppe rouge, Bufr. XI. p. I 32 . BRiss. Ornith. IV. p. 209. greate Cockatoo Eow. 160. Linn.Gen. 45. o. Lati. 1. p. 257. n. 62.

499. Perriche a tete jaume, Buff. XI. p. $3^{S} 3$. Pfittaca Carolinenfis, Briss. Ornith. IV. p. 350 . Linn, Gen. 45. 13. Caroline Parrot Lath. birds I. p. 227.29. Caresb. Carol. J. pl. II.

500. Le vafa (Perroquer), Burf. XI. p. I66. Pfittacus Madagascorienfis niger, Briss. Ornith. IV. p. 317. P fittucus niger, .Linn. Gen. 45. 29. Black Parrot, Edw. 1. pl. 5. Latil. b.rds I. p. 264 .

50I. Rollier de Madagascar, Buff. V. p. 202. Rollier des Indes, .Briss. Ornith. II. p. 75. (B) Coracias Ortentalis, ß. Iann. Gen. 51. 4? Madagascar Roller Latir. birds I. P. 4I3. II.

502. 1. Nefange a gorge noire, Burf. X. p. 121. Parus Canadenfis atricafillus, Briss. Omititi. III. p. 553. pl. XXIX. I. Parus atricapillus Linn. I I 6. 6.

2. Metinge huppíc Buff. X. p. 175. Buss. Ornith. III. p. 558. Parits crifatus, Linn. Gen. II0. 2.

3. Mefunge a longue Queue, Buff. X. p. i 6o.pl. IV. Parus longicau-

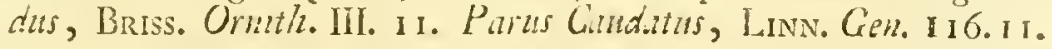

503. I. Le Cochevis (Aloucte), Burf. IX. p. 93. 5. Aluluda crifiata Briss. Omith. III. p. 357. Alandal crifiata Linn. Gen. 105. 6.

2. Le Lulu (Alouette), Buff. IX. p. 105. Petite Alouette huppée,

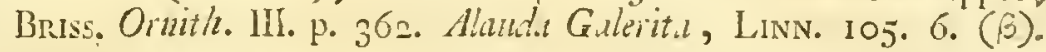

504. I. La grifetre (Alontte) Burf. IX. p. 112. Alouette huppée de Sene-

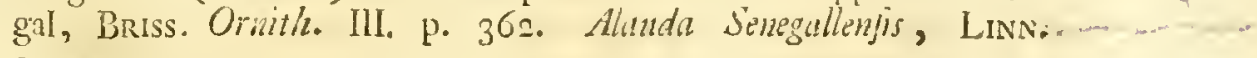
105. 0.

2. Cravatte Jaune, Buff. IX. p. 77. Alauda Capitis bone Spei, Briss. Ormeth. III. p. 364. pl. XIX. 3. Alauda Capenfis, Linv. 105. 0.

505. Petit Coucous noir de Cayenne, Buff. XII. p. 85 . Briss. Ornith. IV. p. 124. pl. XVI. 2. Linn. Gen. 57. I 4. $\beta$.

506. I. Le Baltimore Buff. V. p. 318. Briss. Omith. II. p. I09. pl. XII. 1. Oriolus Baltimore, Linn. Gen. 52. Io. Balcimore oriole Lath. birds 1, p. 432, 19. 
2. l.e Baltimore batard, Burf. V. p. 321 . Briss. Onith. Its p. 3 , Oriolus fpurins, Linv. Gen. 5q. 11. Bultard Buitimore oriole, LatH. lord's. P. 433 . $=0$.

50\%. Nerie huppé de la Chine, Burs. VI. p. 5.3. Bruss. Otmith. II. p. 253. Gracula Criflatella, Imsn. Gen. 53. 5. Chinctehe Saring, EDw. I. 19. Crelted grakle I aTH. birds p. 464.7.

508. Petie Nerle huppé, Je ne le trouve poine chet. N. Burfon.

509. Pic Jaune de Cayenne, Buff. XIll. 48: Micus Cavanentus aléns Busss.

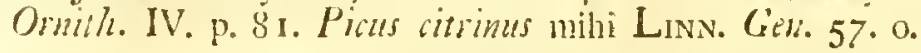

5 10. Le Bifet Burf. IV. p. $3^{11}$. Colunba Vinggo, Briss. Ornitia. I. p; 86. Columba Oenas, Linn. Geis. iof. I.

511. I. Ortolan de I orranc, Buff. VTlI. 29: Briss. Omith. o. Enleriza Migruiz, Lunv. Gen. 110. o.

2. Ortolan de Pasfage, Femelle du precedent.

51 . Coucon noir de Cayenne, Burs. Xill. p. 35. Cuculus ater milhi Lisv. Gen 110. O.

513. Rousferolle Brffr. V. p. 405. Briss. Ornith. II. p. 219. pl. XXII. ¿. Turdus artindis aceus, Linn. Gch. 107. 25.

514. Perroquet verd. Buff. XI. p. IGI. Pfattalus fmen/ls, Briss. Ornith. IV. p. 291. Linn. Gen. 45. O. green and red Chince Parrot, Edw. glean Tab. 231. Lath. birds I. p. 278.85.

515. Litorne de Cayenne, Buff. V. p. 425. Briss. Ornith. II. O. Lisn. Gen. 10:.0.

516. Merle a plaftron blanc, Burf. VI. p. 15. Merle a Collier Briss. Onitit. II. p. 235. Turdus torquatus, LinN. 107. 23 .

517. Perruche a Mouftache, Butr. XI. p. 207. Pfittaca Rriss. Omaith. IV. o. Pfittacus viobrifa, Linn. Gen. 45. O. Multachoe Parrakeet, Latho birds p. $=38.3^{8}$.

518. I.ory cramoifi, Buff: XI. p. 185. Lory d'Amboine, Briss. Ornith. IV. p. 231. Pfitacus Cardinalis, Linn. Gen. 45. O. Crimfon Lory, Latu. birds I. p. 273 . n. 79.

519. Lory rouge Buff. XI. p. I 86 . Briss. Ornith. IV. O. Linn. 45 O. Molicial Lory, Latu. birds I. p. 274.80.

520.hasfigs/.42. 520. Coulacisfi (Perruche). Burf. XI. p. 236. Pfuttacula Philippenfis, Briss. Ormití. III. p. 392. pl. XXX. 1. Pjutacus Galgutus, B.LinN. Gen. 45. 46. Lath. Blids I. p. 3I I. n. I 19. Philippine Parrakect.

521. Choucas chauve de Cayenne, Burf. V. p. 95. Briss. Ormith. II. p. 24. 6. Corvus monedala, L.inn. Gen. 50. 6. Bald Crow. Laris. birds I. p. $3^{8} 3.16$.

522. Chouc Burf. V. p. 101. Choucas noir Briss. Ormith. IV. p. 28. Lins. Gen. 50. o. Biack Jackdaw, Lati. birds I. p. 379.9. C.

523. Choucas Buff. V. p. 95. voyez pl. 52 I. Briss. Ornith. II. p. 24. Coryus monedula, Lins. Gen. 50. 6. Jackdaw Lath. birds I. p. 378. 9.

524. I'ic mordoré, Burr. XIII. 51. Briss. Ornith. o. Picus fusio fulvus, LiNN. 57.0 .

525. Gun rouba (Perruche), Buff. XI. p. 380. Pfittaia Brafilienfis lutea, Briss. Ornith. IV. p. $3^{69}$. Pfitacus luteus, mili Lonv. Geir. 45. 0. yellow Patrot Latri. birds p. 225. 28 .

526. Papegay maillé, Bcte. XI. p. 164. et 335 . pl. XII, Perroqutet varié, 
P1.

Buss. Oiwith. IN. p. 300. Pfttacus aicipitrimes, Linn. Gear $45 \cdot 38$. maillcd Parrot LAtri. I. p. 267 .

527. Perruche Maipourri Buff. XI. p. 350. Pfittazes Mexicanus pectore albo, Briss. Ornith. IV. p. a9ษ. PFittacus melanocephalus, LiNN. Ge\%. 45. 33. Pfittaizs atricafillus, Mirler pl. 4. A. Whithe breated Parrot, Edw. IV. p. 169. Lath. lird. I. p. 305. n. 112.

528. Aputé Juba, Buff. XI. p. 376. pl. Xill. Perruche Ilinoife, Rriss. Or-

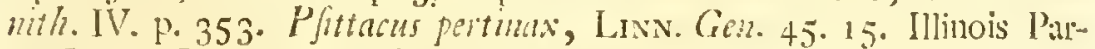
rot Lati. I. p. 228. 30. EDW. 23t. yellow faced Parrakeer.

529. Geay blet de l'Amerique Septentrionale, Bums. V. P. 165. Geay bleu de Conada Briss. Ormth. I. p. 55. Consus irifiatus Lins. 50.8. Blue Jay Lati. birds I. p. 386. 20. Enw. 239.

530. Geay brun de Canada, Bufron V. 1. 160. I3riss. Omith. II. p. 54. Corrzus Cilludenfis Linn. Gen. 50. 46. Latu. bards p. 389. 11. 23.

531. Choquard Bufr. V. p. 105. Choucas des Alpes Briss. Ormith II. p. 3o. pl. 1. .. . Cormes pyrrocorax, LinN. 50. 17. Alpine Crow. LATH. birds I. p. 381 . 11.

532. Troupiale Burf. V. p. 277. Briss. Oruith. II. p. 86. pl. VIII. r. Oriolus 7cterus, Linn. Gen 52. 4. Jeteric Oriole LatH. brids I. p. 42. 6.

5.33. Troupiale a calotte noirc, BuFr. V. p. 505. Troupiale brun de la nouvelle Espagne, Briss. Ornith. II. p. 105. Oriolus mexicanus, Linn. Gen. 52. 8. Black crowned oriole Lat11. p. 431. I6.

534. Troupiale noir Burf. V. p. 301. BRIss. II. p. 103. Oriolus niger mihi Linn. Gen. 52. o.

535. 1. Carouge Buff. V. p. 335. Briss. Ornith. II. p. 216. pl. XII. 2. Oriolus Bowana, Linn. Gent. 52. 11. 12. Bonana oriole, Lath. birds I. p. 456. 22 .

2. Carouge de l'ile St. Thomas, Burf. V. p. 342. Briss. Ornith. IL, p. 123 .

536. Troupiale de la Gujane, Buff. VI. p. 299. Briss. Ornith. II. p. Io7. pl. XI. I. Oriolus Gujanenfis, Lisn. Gen. 52. 9? Gujana oriole, Lathas birds I. p. 430.15 .

537. Titiri ou Piperi, Bufr. VIil. p. 389 . le Tyran Briss. Ornith. II. p. 391. Lamus Tyramines, Linn. Gen. 44. 13. Tyrane Thrike, Lathau berds I. p. 184 .

538. Pie du Senegal, Burr. III. p. 133. Briss. Omith. II. p. 40. pl. III. f. a. Corrus ater Linv. Gen. 50. 12. African Crow. Lath. birds I. p. 394. 3 I.

539. 1. Palmifte (grive), Bufr. VI. p. 103. Briss. Ornith. II. p. 303. pl. XXIX. fig. 1 2. Turdus Palmarm, Linn. Gen. 107.19.

2. Soui Jala, Buff. VI. p. 99. Merle doré de Madagascar, Briss. Or. with. II. p. 247. pl. XXIV. 2. Turdus Fall, LinN. Gen. 107. o.

570. Merle viole:, Burr. VI. p. 63. Briss. Ormuth. II. o. Turdus Fuide, Linv. Gen. I07. 0.

541. Lc Kinki Manou (Gobemouche), Burf. VIII. p. 409. grand Gobemouche cendré Madagasenr, BRIss. Ornith. II. p. 389. pl. XXXVII. I. Mufcicapa kinki, Lins.

543. 1. Hirondelle de Cheminée, Buff. XII. p. 238. pl. X. Briss. Omith. 11. p. 486. Hirmado rufíca, LiNs. Gen. 117. I.

$\mathrm{H} 2$ 
2. Itrondlle de Rivage I3rre. Xit. p. 383. Briss. Onith. II. p. 506. Hhindo rituld, I.1N. Gen. 117.4

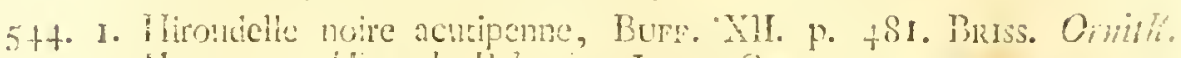
11. p. 50:. Mlimindo Pclugga, Linv. Gien. io.

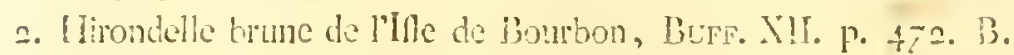

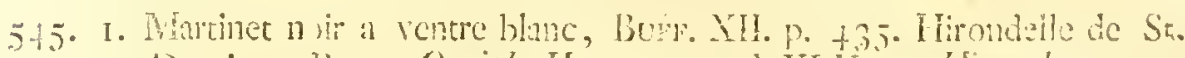

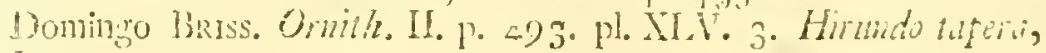
LINN. II - 9 .

2. Hirondelle de Caycne, BefF. XII. p. 44.3. I. Hirondelle de Cayenne

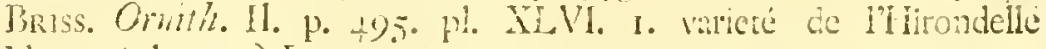
beue, ipl. 722.) Linn. in 7 . 0 .

5t5. I. Hirontelle a ventre tacheté de Cayemne, BuFf. XII. p. 453 . Buss

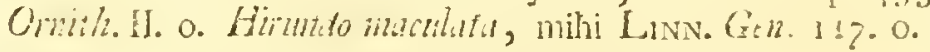

2. Tirondelle a ventre blane de Cayenne, Burt. XII. p. 451. Bniss,

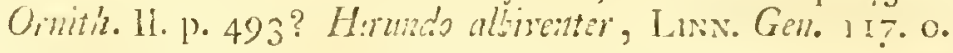

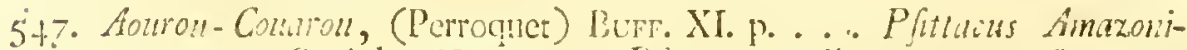

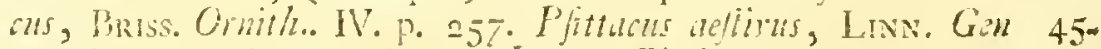
32. Common Amazon Parrot, LATil. Birds I. p. $28 \% .91$.

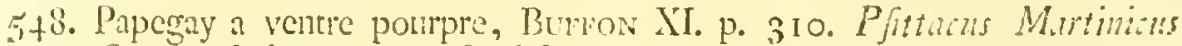
Gyarocephalns, Briss. Omiti. IV. p. 25I. Line. 45. o. Aslicrowned Parrot, birds I. p. 281. n. $88 . \mathrm{C}$.

549. Perroquet Amazone a tete blanche, Burf. XI. p. 299. Pfittucus mum timicanus, Buss. Ormith. IV.p. 212. Pfottucus leucocephulus, Lixw. 45. 30. Wirhe headed Parror, Latr. livds I. p. 280. n. 88. A.

550. Perruche Sincialo, Betf. XI. p. 370. Pfittuca..... Bruss. Ormith. IV. p. 319. Yjittacus rutrofitio, L.nN. 45. 18. long tailed green Par= rakeet, EDw. [V. Tab. i75. Latit. birds p. 230. 11. 33.

55I. Pertuche a Collier conleur de rofe, Buff. XI. p. 212. Pfituca lorquatu, Briss. Omith. IV. P. 323. Pfotacus torquatus, LinN. 45. O. Rofe ringed Parrakee, LATu. lirds I. p. 235 . 37.

552. Perruchic Lory, Burr. XI. p. 202. Pfithaca Indica varia, Briss. Ornith. IV. p. 366. Pfitactis ormatus, Lins. Gon. 45. 19. Lory Parnkect EDw. p!. 174. Latr. lirds p. 221.11. 24.

553. Pic varié ondé, Buffon XIII. p. 117. Pic varié de Cayome. Barsa. Ornith. IV. p. 54. Picus tridactylus, Lins. Gen. 59. 21. Souchen trie toed woodpecker LaTn. birds p. Go1. 51.4.

554. I. Motreux on Culblane, Bufr. IX. p. 341. Culblanc ou vitrel Briss. Onith. Ili. p. 449. Motacilla oeninthe, Livn. I I to 15.

2. La Fenclle.

555. Femelle du Micrle.

556. I. Litorne de Camada, Butf. V. p. 426. 2. Grive de Canada Briss. Omith. II. p. 225. Turdus megratorius, Linv. 107. 6. Catesb. I. 'Tab. 29.

2. Merle brun ou Grivette d'Amerique, Buff. Y. P. 400. MInuvis de la Caroline Briss. Ornith. II. p. 2! 2. Tudus bumueus, mihi Liss. Ge\%. $10 \%$.

55\%. T. Thanambé (Cirive) Buff. VI. 8ı. Merle de Madagascar, Briss. Ormith. II. p. eg1. Tudus Madagarcurienlis, Linn. 10\% 0.

2. Omormer Butr. VI. p. 7n. Merle cenitré de Mladagascar, Briss.

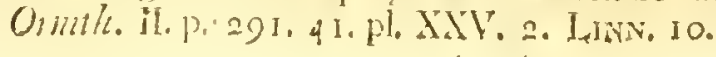


Pl. $(33)$

558. 1. Le Moqueur ou Merle cendré de St. Domingo, Burf. V. 15. 4.5\% Turdus mimus Briss. Ormith. I1. 1. 262. Turdus polyglotius, LiNN. 10\%. 10.

2. Merle olive de Cayenne, varieté du Mocqueur:

559. Merle a gorge noire de St. Domingo, Beff. VI. p. 76. Briss. Ormith. o. LINN. o.

560. 1. Le Tilly Burr. V. p. 457. Grive cendrée d'Amerique Briss. Omith. II. p. 288. Turdus plumbeus, Linv. Gen. 107.10.2

2. Merle a cravatte de Cayenne, Bufr. VI. p. 90. Briss Cruit/2. II. o. Linn. Gen. 107. 0.

561. Merle verd d'Angola, Buffon VI. p. 6i. Briss. Omith. II. p. S11, pl. XXX. 2. Turdus nitens, Lins. 107. I 3.

562. Merle de Roche, Buff. VI. p. 31. Briss. Ornith. II. p. 238.240. Turdus Saxatilis, Linn. 107. I5. Rock fhrike Latnas birds 1. E. 176. n. 27.

563. 1. Merle huppé du Cap de bonne Esperance, Berf. VI. p. yI. Briss. Orimth. II. p. 257. pl. XX. 2. Turdus Cafer, Lin'v. Gü. 107. 16.

2. Merle brun du Senegal, Burf. VI.p. 80. Beuss. Omith. 11. p. 261 . Timdus lugubris, mihi Lin. Gen. 107. 0 .

564. Merle olive des Indes, Buff. VI. p. 78 Briss. Ornith. II. p. $29 \%$ pl. XXXI. 2. Turdus olivaceus, milii Lins. Gen. 107.0.

565. 1. Gobemouche, Buff. VIlI. p. 325. Briss. Ornith. II. p. 35\%. pl. XXXV. 3. Mufcicupa Grifola, Linv. Gen. I 13.20.

2. Gobemouche noir a Collicr, Burf. VIII. 1). 308. Gohemouche nois Briss. Grmith. II. F. 381 . Mufricufu atiliatpilla, LInn. Geit. II 3.9.

3. Varicté blanche de 675 . i.

50̌6. 1. Petit Gobemouche noir Aurore, Burr. VII. P. 3.9. Cobcunouche d'Amerique, Briss. Ornith. II. p. $3^{8} 3$. Mulcrougu Ruticilta, Lisw. Geth. II3. IO.

2. Sa Femelle.

567. 1. Gobemouche a bandenu blanc Burr. VII\%. p. 320. Gubemouche a

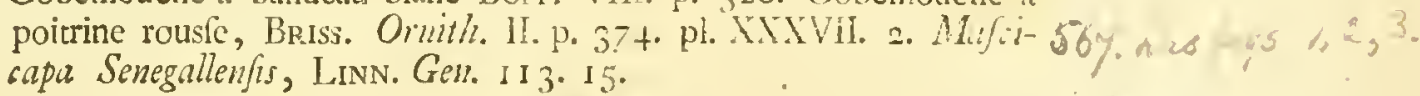

2. Varieté a poitrine noire, Briss. Gmith. II. p. 376 . pl. XXXVII. 3. LINN. Geh. II 3. I5. B.

568. 1. Gobemouche huppé de la Martinique, BefF. VIII. p. 3to. Briss. Ornith. II. p. 362. pl. XXXVI. 2. LINN. Gel. $113 \cdot 3 \cdot$

2. Moucherolle brun Buff. VIII. p. 374. Briss. Oinith. II. p. 364 . Mufsicapa fusca, mihi Linn. Gen. 113 . o.

569. I. Moucherolle a huppe verte, Buff. VIIT. 1. 379. Gohemouche huppé de la Virginic, Briss. Ornith II. p. 41 ฯ. Mufcicafu crinitu Lins. Gen. II 3. 6.

2. Gobemouche a ventre Jaune, Eurr. VIII. p. 355. Gubemouche de Cayenne, Briss. Ornith. II. p. 40.4. pl. XXXVIII. 4. Matsiciat Cayanenfis, Linv. Gen. II 3.12.

570. Couliavan (Loriot) Busf. VI. p. 360. Loriot de h. Cochenchine, I 3 Bess. 
I.

\section{(. 34$)$}

Briss. Omith. II. p. 326. Oriolus Cheneiffs mihi Liws. Gen. 52. a

Latinam birds I. p. 452. 43. C.

57 I. I. Tyran de Cayenne Buff. Vili. p. 404. Brisson Oriath. II. p. 398. Mufcicupa antrora, Linn. Gen. II 3 -o.

2. La Savana, Buff. VIII. p. 365. pl. XIII. Tyrn a queue fourchue Briss. Onniti. If. p. 396. Mufcicapiz Tyranmus, F. Linn. Gen. II 3 . 4. B.

572. I. Gobemouche du Cap. Bufr. VIII. p. 317 . Bruss. Ornith.'II. p. 372. pl. XXLVI. 3. Muscicapa Capenfis, Linn. Gen. I1 3. 17.t

2. 2 ag. 2, 9. 2. Gobemouche a Collier du Cap, Burron. VIII. Briss. Ornith. II. p. 379. p]. XXXVI. 4. Mufcucapa toiquata, I.InN. Gen. 113.17.

$5.3 \cdot 1$. Gobemouche huppé de l'Ille de Bourbon, Burf. VIII. p. 425. Briss. Omith. It. P. 420. 11. 32. pl. XXX1X. 5. Linv. Gell. I1 3. O.

2. Gobemouche huppé de Senegal, Burs. VIII. Briss. Oinith. II. p. 422. LINN. Gen. II3. O.

5. +. 1. Fobemonche brin, Buff. ViIl. p. 333. Briss. Ornith. II. o. Mis. fitapa filsa, LINN. II3. O.

2. Gobemouche olive Bufr. Vill. p. 346. Briss. Ornith. II. o. Mitfercupa diva, Linn. Gen. 113 . o.

3. Gobemouche a Poitrine rachetce de Cayenne, Bufr. VIII.p. 347. Bkiss. Ornith. II. o. Linv. Gen. I [3. O. Muscicapu naeria milhi.

3 figs. 375. 1. Varieté de INo. 4.

2. Grimpereau foui manga. Angala Dian, Burf. X. p. 265. Grimpe. renu verd de MIadagascar, .Busss. Ornith. 1II. F. 64I. Certhız Lothenia, Jinn. Gen. 65. 25. Lothens Creeper, Lati. Uira's I. p. $715 \cdot 13.16$.

3. Sa Femelle.

4. Soui manga olive a gorge pourprée, Burf. X. p. 260. Grimpcreau olive des Philippines, Briss. Ornith. III. p. 623. nl. XXXIV. 4. Certhia Zeilonica, Linn. Gen. 65. 23. Zuiloneze Crepä, Litit. birds I. ?12. 13.

5.6. I. Soumanga olive pourpré, BuFF. X. p. 260 . varicté du precedent, Celhia Philippenfis, Briss. III. p. 613. Certhia Philitpina, Lisx. Cien. 65. 21. Philippine Creeper, Lat11. birds I. p. 711. 1. 12.

2. Vinieté de la precedente, Grimpereau gris des Philippines, Briss. Ornith. III. p. 615. pI. XXX. fig. 3. Certhia Curvisaria, LINN. Gen. 65. 6.

3. Aurre varicté, Buff. X. p. 267. Briss. Ornith. III. p. 6I 6. pl. XXXIt. 5. pecit Grimpereau des Philippines, Certhia 7 ugularis, 1.iNn. Gth. 65. 7. Grey Creeper, Lath. birds I. p. 71 4. n. 15. A.

7. Trareté da Souimanga olive a gorge pourpré, Buff. X. p. 261 .

5.7. Ko lili (Toucan) Buff. XIII. p. 192. Toucan a Collier de Cayenne, Bkiss. Orwith. IV. p. 429. Ramphafius piperivorus, Livn. Gez. 46́. 2. Piperine Toucan, Jatham birds I. p. 334. I1.

5.-3. 1. Guitguit vert et bleu a gorge blanche, Buff. X.p. $3^{\circ}$ s. 2. Grimpereau verd du brefil, Bruss. Ornith. III. p. 631. Certhia faiza, Lisn. Gen. 65.12 . Blue lieaded Creeper, Latru birds I. p. 727.11 .28 . D.

2. Cuitguit verd er bleu a tete noire, BufF, X. p. ३300. Grimpereas 
11.

\section{$(35)$}

yerd a tete noire du Biefll, Beiss. Omith. III. p. 633. Certhin Jpiza, Linn. Gell. 65. 12. black handed Cresper, Lath. birds I. p. $727 \cdot 17.28$. A.

3. Oifeau brun a bec de Grimpereau, Burr. X. p. 287. Grimpereau noiratre du Brefil, Briss. Ornithe p. 658. pl. DXXIII. fig. 3. Certhia guturalis, Linn. Gir. 65. i5. Latno lizds I. p. 723. n. 24. green faced Crceper.

579. 1. Fatrette Buff. IX. p. 163. pl. VII. Curruca Briss. Ornith. III. p. 372. Motailla hippolals, Lins. Gen. 1 I $4 \%$

2. Pasferinette Buff 1X. p. I7S. Petice fauvette, Briss. Orinth. IlI. p. 374. Motacilla Borin, mihi Linn. I 4 . o.

3. Grifette Buff. IX. p. I9I. Curruca Gineraria, Brass. Ornith. IIf. p. 376. Motacilla Jylvia, Linn. 11 4.9.

58c. I. Fauvette a tete noire, Burf. IX. p. I8 1. Briss. Ornitiz. III. p. 384. Motacilla atricatilla, Linn. Gell. I I f. I 8.

2. Sa Femelle.

3. Fauvette babillarde, Buff. 1X. p. 195. Carma Garrala, Briss. Oriath. III. p. $3^{84}$. Motucilla Curruca, Linn. Gen. 11 is 6.

591. I. Fatuvette rousfe, Buff. IX. p. 2 I I. Cutrucar mifa, Briss. Orilith. III. p. 387 . Motacilla rufa, mihi Linn. Gen. i I 4 . o.

2. Fauvette des Rofeaux, Buff. IX. p. 206. Cairalid armdina:ea, Briss. Ornith. III. p. 378. Motacilla Salicaria, Linn. II 4. 8.

3. Fauvette tacheté, Burf. IX. p.215. Curruca naevia, Briss. Ornith. III. p. 389. Motacillu nuevia, Linn. Gen. I I 4. o.

582. I. Figuier de Senegal, Burf. IX. p. 410 . Ficdula Senegalenlis, Briss. Ornith. III. o. Motacilla Scnigallenji, Lins. Guil. 1:4. 0.

ュ. Premicre mieté.

3. Autre varieté.

583. I. Traquet de Senegni, Bufr. IX. p. 3a8. Ruthotra Senegulenfis, Briss. Orwith. Ill. 1. iti. pl. XX. 3. Linn. Geh. II 4 o.

2. Motteux de Senegal, Burf. IX. p. 359. Briss. Cminta. UI. o. Linr. Gen. II 4. o.

584. 3. Figuier brun de Senegnl, Je ne le trouve point chez M. Bufroy.

2. Premiere varicté.

3. Seconde varieté.

5โ5. 1. Todier de I'Amerique Septentriomie, Bur. XIII. p. 33r. Todier Briss. Ornith. IV. p. 528. pl. NLI. 2. Todus viridis Linv. Geia. 6i. 1. green Tody Lath. berds I. p. 65t-1. Pennant Gen. of birdis p. 62. pl. 6. I. green fparrow. Eow. bin ds II. I2 I.

2. La Femelle.

3. Le Tic tic (Todier) Bufr. XTI. Todier cendré Briss. Omith. Juppt. 134. Todus Cinereus Linv. 61. 2. Cinereous Tody Latn. bisds I. p. 658. 2. grey and yellow Flycatcher, EDw. birds pl. 262.

586. Boutfallick (Coucou), Buff. XII. p. 24. 7. Coucou tacheté de Bengale, Briss. Ornith. IV. p. I32. n. I3. Guculus fcolopaceus, Linn. Gen. 57. I I. Indian fpo:sed Cuakow, Late. birds I. p. 5I6. n. 6. EDw. birds pl. 59.

587. Yourou Driou (Coucou), Burf. XIJ. p. 55. Gुrnd Coucou de Mada- 
PI.

gascar (male), Briss. Ornith. IV. p. 160. Cusulus aeneus, Lisv. 5?. o. africall Cuckow Lati. birds I. p. 532. 30 .

588. Sa Femelle.

589. Coua (Coucou), BufF. XII. p. I 5. pl. I. Coucou huppé de Madagas. car, BRIss. Ornith.IV.p. 149. pl. Xil. fig. 2. Cucculus criftatus, Linv. 57. 12. Madagascar crelted Cuckow LATH. birds I. p. 522. I5.

590. Martin pecheur a grosbec, Burf. XIII. p. 271. Ispida Capitis bone Jpei, BrIss. Ornith. IV. p. 488. pl. XXXVI. 3. Aliedo Capenfis, LINN. 62. 9. Cape kingfisf her, Lati. birds I. p. 610. 2.

591. Martin Pecheur vert et blanc, Bufr. XIII. p. 318. Briss. Ornith. IV. o. Linn. Gen. 62. o. White an green Kingtisher, Lath. I. p. 641.30.

592. 1. Martin pecheur vert et roux, Buff. XIII. p. 317 . Ispida Briss. Oruth. IV. o. Alcedo siridi rufa, mihi LinN. Gen. 62. o. Rufous and green Kingfisher; LaTH. birds 640.29.

2. Sa Fcmelle.

593. Jaguacati (Martin pecheur), Burf. XIII. p. 512. Ispida Brafilienfis cristata, Briss. Oinith. V. p. $5^{\mathrm{I} 1 .}$ 20. Ispida Carolinenfis criltalla ibid p. 5 I 2. Alcedo Alcyon $\alpha . \beta$. Linv. Gen. 62. 7. Belted kingfisher Latif. firds I. p. 639. n. 27. B. American Kingfisher, EDw. birds IIl. pl.

$59+$ Martin pechcur a tete grife, BuFf. XIII. p. 286. Ispida Senegalengis major, Briss. Ornith. p. 494. pl. XL. 1. Alcedo Senegallenfis, LiNN. Geir. 22. 17. Crab eating kingfisher, LathaM birds I. P. Gr8. n. 9. $A$.

595. Epeiche ou Pic varié, Burf XIII. p. 85. Picus rarius matior, Briss. Ormith. IV. p. 34. Picus major, Linv. 5\%. 17. greater fiotted woodpecker Lath. birds I. p. 564. (voyez le male pl. 196.)

596. Pic noir Buff. XIIL. p. 62. pl. 2. Picus niger maximils, Pierss. Ornith. IV. p. 21. Picus martius, Linn. Geis.59. I. great black woon. pecker, LaTH. birds I. p. 552. n. I.

597. Pic varié de la Jamaique, Bufr. XIII p. 105. Briss. Ornith. IT. p. 59. Picus Carolinus Linn. Gen. 59. ro. Carolina woodpccler, Lairus. Birds I. p. 570. n. I7.

598. Petice Epeiche Bufr. XIII. p. 93. Picus varius minor, Briss. Omitls. IV. p. 41. Picus minor, LinN. 59. 19. lesfer fpotted woadocclici, LaTH. birds I. p. 566. 14.

599. I. Le Colibri Topaze, Buff. XI. p. 63. Polytmus longicaudus ruter, Briss. Omith. III. p. 690 Tiochilus Pella, Linn. Gen. 66. = Topaz humming bird, LaTH. birds I. p. 74. 7ః. Longtailed red hummingbird, EDw. birds I. pl. 32 .

2. Colibri violet, Buff. XI. Colibri violet de Caycnne, BrIss. Ornith. III p. 683 . n. I1. pl. 353 . violet hummingbird, LATI. birds I. p.
756 . I 5.

6co. I. Le petit Colibri, Buff. XI. p. 88. Polytmus Briss. Ornith. III. p. 667. Trochilus Thammatias, LINN. 66. 8. admiable hummingbird, LATH. birds I. p. 763. 25 .

2. Colibri violet, Buff. XI. p. 79. Polytmus Cayanenfis trolaceus, 13k1ss. Ornith. Ill. p. 683 . pl. XXXV. 3. Trochilus Jugularis,

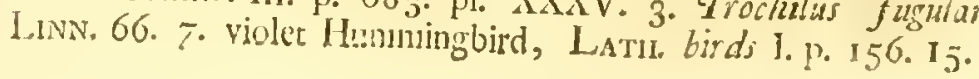


P].

\section{$(37)$}

3. Le Brin blanc, Buff. XI. p. 67. Polytmus Cayanenfis longicaudus; Briss. Ornith. III. p. 686. Trochilus Juperciliofus, LinN. 66. 3 . fupcrcilious humming bird, Latri. birds 1. p. $747 \cdot 3 \cdot$

4. Collier ronge Burf. XI. p. 8I. Polytmus surinamenfis, Briss. Ornith. IlI. p. 674. Trochilus leucurus, Lins. 66. 6. White tailed hummingbird, Latr. hirds $757 . \mathrm{I} 7$.

60 I. Le Touraco, Buff. XI.p. 419. pl. 15. Coucou vert huppé de Guinée, Briss. Ornith. IV. p. 152. Cuculus Perfa, Linn. Gen. 56.17 . Touraco Cuckow, LATH. birds I. p. 545. 46.

602. Le Barbican, Buff. XIII. p. 197. Briss. Ornith. o. Lins, o. doubtful Barbet, LATH. birds I. p. 506. I5.

603 . Le Balicasfe, Buff. V. p. 115. Corbeau noir verdatre, Bkiss. Ornith. II. p. 31. pl. 2. fig. I. Corvus Balicasfuts, LivN. 55. I1. Philippine Crow. Lati. bird's I. p. 38 I. I2.

6a4. Merle de la Chine, Buff. VI. p. 1 I5. Merula Briss. Ornith. o. Linn. o.

605. Pic Grimpcrean ou Talapiot, Buff. XIII. p. I22. Briss. Ornith. o. Linn. o. climbling Oriole, Latil. birds I. p. $45 \hat{3} .45$.

606. I. Petit Troupiale noir, Bufr. V. p. 303 . Briss. Ornith. II. p. Iо3. pl. XI. Linn. Gin. 52. o. Oriolus ater, black oriole Lath. birds J. p. 445. n. 37 .

2. Troupinle olive de Cayenne, Buff. 309. Briss. Ornith. III. o. Cayenne olive oriole, Lath. birds I. p. 447. n. 39.

60\%. I. Femelle du Troupiale noir Chesnuth and black oriole, Latr. birds I. p. 437.12 .24 .

2. Carouge olive de la Louifanic, Burf. V. p. 345. Briss. Ornit II. p. I28. Oriolus Capenfis, Linn. Gen. 52. I 8. Olive oriole Lat11. birds I. p. 444. 11. 35 .

608. Geny de Sibcric, Berr. V. p. I6r. Briss. Ornith. o. Corrus Sibericus mihi Linv. Gen. 5 r. o. Siberian Jay, Latu. kirds I. p. 39 г 26.

609. Colnud Buff. V. p. ir 4. Briss. Ornith. o. Corsus nudicollis Linn: Gen 51. о. Bareneck Crow. Lath. livds I. p. 332. I5.

610. 1. La Gorge bleve, Burf. 1X. p. 300. pl. XII. Ficedula Cyanacula, Briss. Ornith. III. p. 413. Motucilla Suecica, Linv. Gen. II4. 37. R.

2. La Femelle.

3. Le Jeunc.

611. Varieté de l'Epeiche ou pic varié, pl. 595 .

61 2. Pic a col rouge, Buff. XIII. 8o. Briss. Ornitho o. Linv. Gent. 59. o: P'icus rubricollis, mihi. Red necked woodpecker LaTı. birds I. p. $55^{8} .5$.

51 3. Petit Pic rayé de Cayenne, Buff. XIII. p. 46. Picus Cayanenfis firintus, Briss. Ornith. IV. p. 69. Lins. Gen. 59. O. Picus punotigula Caycnne woodpecker, LATH. birds I. p. 590. 40.

6 I4. Petit Pic rayé de St. Domingo, Buff. XIII. p. 46. Briss. Ornith. IV. p. 67. n. 26. pl. III. 2. LinN. GEn. 59. o. Rayed woodpecker LatM. lirds I. p. $5^{88 \mathrm{n}}$. $3^{6 . A .}$

6I5. I. Traine buisfon, Buff. IX. p. 2I8. pl. IX. Currua fepiaria, Briss Ornith III. p. 349. Motacilla modularis, LInN. II 4 . 3 . 
P1.

2. Le Rosfignol. Buff. IX. p. I15. Lafimia Briss. Ornith. III. p. 397. Motacilla Lafcinia, LinN. I14. I.

616. Le Grivert, Burf. V. p. I83. Briss. Ornith. o. Coracias Cayanus, Linn. 51. o. Cayenne Roller, LATH. Birds I. p. 11515.

617. Le Kink, Buff. V. p. 347. Jcterus Briss. Ornith. o. Oriolus Linn. U.

618. I. La mouftache (Mefange), Bitr. X. p. 135. Mefange barlute, Briss. Ornith. III. p. 567. Parus biarmicus, Linn. Gen. Ii 6. 2 II.Sa Lemece.

3. Le Remiz, Buff. X. p. I41. Mefinge de Pologne, Briss. Omith. III. p. 565. Parus pendulinus, Lins. Gen. 116.13 .

619. Rollicr des Indes, Burf. V. p. sor. Gulgulas Indicus, Eriss. Ornith. 11. p. 75 . pl. Vli. 2. Coracias orlentalis, Linn. Gen. 5 I. 4. Oriental Roller, Latu. birds p. 4I1. 6.

Gan. Rolier de la Chine, Buff. V. p. i 8 I. Briss. Ornith. II. p. 7\%. pl. VI. fig. 2. Coracias chimenfis, Linv. Gtn. 5 I. O. Chinefe Roller, Lath. birds I. p. 4I 4. n. I4.

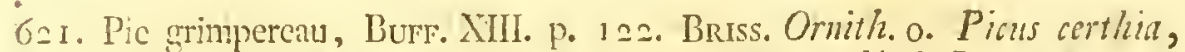
milin Linn. Gen. 59. o. Climbing Gakle Lath. birds I. p. 467. I1.

622. Geny de la Chine a bec rouge, Burf. V. p. 157. Briss. Ornith. o. Corrus erythrorynchus, mihi LinN. O. Red billed Jay, Latu. birds 390.24 .

623. 1. Sitelle ou Torchepor, Burf. X. 1\%. 206. fitta Briss. Oimith. III. p. 583. Jitta Europaea, Linv. Gen. 60. I.

2. Sitclle de Cinnada, Buffon X. p. 209. Bkiss. Orniti. IIT. p. 393. fitu Canadenfis, Linn. Gen. 6o. 2.

624. Le Qnereiva, Buffon VIII. p. 197. Cocinga Cayanenfis, Rerss. Ornith. II. p. 344. Ampelis Cayma, Linn. Gen. 108. 6.

625. Gcay de Peru, Burf. V. p. 158. Briss. Ornith. o. Cormus yncus mihi Linn. Gei. 52. o. Peruvian Jay, Lath. birds I. p. 391. 27.

626. Rollier d'Abysfinie, Bufr. V. p. 125. Briss. Omith. o. Coratias alys. finus, Linv. Gen. 51. o. Abysfmin Roller, Latu. lirds I. p. 408.2.

627. 1. Merle de Mindanao, Butr. VI. p. 83. Briss. Omitho o. Lunv. Gen. 107. 0. Turdus Mindunenfis, mihi.

2. Micrle Dominicain, Burr. VI. p. 96. Briss. Ornith. o, Lins. o. Turdus dominicanus, mihi.

628. Le Castican, Buff. XII. p. 199.pl. VII. Briss. Ornith. o. Ramphaftos Carsicus, mihi Linn. O. Pied Roller, Latio bird's 1. p. 415. 11. 16.

629. Choucas de la nouvclle Guinée, Burf. XII. p. 199. Briss. Omnth. o. Linn. 52. o. Corsus ftriatus, mihi. New Guinca Crow. Lath. birds I. p. $3^{8 \mathrm{r}} \cdot 10$.

630. Le Choncari, Butr. V. p. in 2 . Briss. Omitho o. Linn. Geil. 5 1. o. Papican Crow. Latio. Birds. I. 382.14.

631. Nanucode a Bouquets, Burf. V. p. 227. Bruss. Ornith. o. Linn. Gen. 540. Paradifea Jpeciola, mihi. maguificent Parradife bird Lath. birds I. p. $477 \cdot$ pl. 19. 17. 3 .

632. Manucode noir, Buff. V. p. 2.31. Briss. Ornith. 0. Linn. Gen. 52.

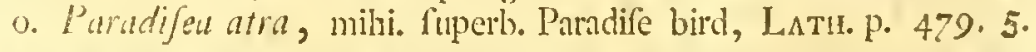

533. Le fix filet, Burf. V. p. 253 . Briss. Ornith. o. Liva. Gen. 52. o. 
Paradifer fexpennis, mihi gold breafted Paradife bird Lath. birds i. p. $48 \mathrm{r}$.

634. I.e Chalybé de la nouvelle Guinée, Burf. V. p. 236 . Briss. Ornith. o. Linv, Gell. 52. o. Mamucodia chalybea, milhi. Blue green Parndife bird LATH. I. p. 482 . 7 .

635. Veuve a cpaulettes, Buff. V'II. p. 232. Briss. Ornith. o. Linn. Gen. 110. o. Emberiza Progné, mihi.

636. Merle Soliraire des Manilles, Buff. VI. p. 48. Briss. Ornith. O. Linn. Gen. 107. o. Turdus manilla, voyez la femelle pl. $5^{6}$. 2.

637. Promerops brun a ventre tacheté, Burf. Ornith. XII. p. 156. Promerops Briss. Ornith. II. p. $46 \mathrm{I}$. pl. XLLII. 2. Upupa promerops, LINN. 64.2.

638. Promerops brun a ventre sayć, Buff. XII. p. 159. Briss. Ornitho o. L.rnn. 64. o. Upupa frriata, mihi.

639. Promerops a parements frizes, Buff. Xil, p. I61. Briss. Oruith. o. Upupu Jpeccoja, Linn. 64. o.

640. I. Rubis Topaze (oilenu mouche), Bufr. XI. p. 27. Mellefuga Brafilieifer gutture topafuno, Briss. Ornith. III. p. 699. Trochilus musquitus, Linv. Gen. 66. 14. $\beta$.

2. La Jacobine, Buff. XI. p. 47. Mellifuga Jurinumen/is torquata, Briss. Ornitle. III. p. 71 3. Tiocibilus mellivora, Lins. Gen. 66. 20. 3. Le Hirppe col. Burf. XI. p. 24. Briss. Ornith. o. Trochilus ornatus mihi l.sns. Gen. 66. o.

64 I. Arn rouge, Burf. XI. p. 250. Ana jumaivenfes, Briss. Ornith. IV. p. 189. Pfutacus micao, Linn. Gen. 45. 1. Red and yellow Maccalv. Lati. p. 201.2.

64.2. Grande Perruche a Collici rouge vif, Bufr. XI. p. 199. Pfittaca Indica torquata, Briss. Ormith. V. p. 32G. PJithacus Alexundri, Linn.Gen. 45. 1 4. Ring Parkeet, Edw. pl. 292. Alexanitrinc I'arrakeet Lati. birds I. p. $235 \cdot 36$ :

6+3. I. Le Grilin Grive, Buff. VI. p. I 4 . Briss. Ornith. 0. Linas. Gel?. 107. o. Turdus grifeus mili.

2. Sa Femelle.

3. Le verdin (Grive), Buff. VI. p. I 16. Briss. Ornith. II. pl. XXX? Turdis niteus, Linv. Gen. 107. n. I1. $\beta$.

644. I. Merle roux, Buff. VI. p. 105. Merula rufa, Briss Omith. II o. Turdus rufus, Linn. Gelt. 107.0.

2. Petit merle brun a gorge rousfe de Cayenne, Buff. VI. p. Io6. Briss. Ornith. 0. LinN. Gen. 107. o. Turdus rutgula, mihi.

645. Le Moqueur François, Burf. V. p. 449. Grive de Caroline, Briss. Ornith. II. p. 223. Turdus rufus, LinN. Gen. 10?.9.

646. Casfique, Buff. V. p. 334. Cassicus Briss. Ornith. II. o. Oriclus violuceus, Linn. Gen. 52.0. White headed oriole, Lath. Birds I. p. 422. 11. 4.

$6+7$. Veuve en feu, Buff. VII. p. 235 . Vidua Briss. Ornith. III. o. Firngilla ardens Linn. Gen: I I2. O.

648. I. Merle violet a ventre blanc, Buff. VI. p. 104. Turdus Briss. Or. sith. II. 0. Turdus leucogafter, mihi Linv: Gen. 107. 0. 
Pl.

2. Le Palmifte (Grive), Burr. V. p. 1 42. grive verd olivatre, Briss. Ornith. II. p. 301. pl. XXIX. fig. 1. 2. Turdus palmarum; Lisw. Gerr. 107. 19.

650. 1. Alouette noire, Burf. IX. p. 3I. 2. Briss. Ornith. III. p. 335. B. Alutulu nora, Linn. Gent. 105.0. 2. Ceinture de Pretre, Buff. LX. p. 37. Briss. Ornith. III. o. Line.
Geh. 105. 0.

65I. I. Pouillot our Chantre, Buff. X. p. 32. Aflus BRiss. Ornith. IIT. p. 47\%. Emberiza Trochilus, Linn. Gen. 114.49.

2. Troglodytes, Buff. X. p. 43 . le Roitclet Briss. Ornith. III. ए. 425. Motacilla Troglodytes, Linn. I I 4. 46.

652. I. Lavandiere, Buff. IX. p. 362. Motacilla BRIss. Ornith. III. n. 461. Motucilla alla, Linn. Gen. I I f. I I.

2. Pivotte ortolane, Buff. IX. p. 284. Briss. Ornith. III. o. Motacilla Lann. Gen. I I 4 . o.

653. 1. Le Zizi Buff. VIII. p. 63. Emberiza Sepiaria, Briss. Ornith. III. p. 263 . Emberiza Cirlus, Lis. Gen. I 10.12.

2. Sa Femelle.

654. I. Le Fift de Provence, Buff. IX. p. 283. Alauda Briss. Ornith. III o. Alauda Linn. Geir. 105. o. 65. 1. Pit Chou, Buff. IX. p. 230 . Briss. Ornith. III. o. Linv. Gen. I I.
o. Motacilla undata.

2. Bouscarle, varieté de la grifette, pl. 579. 3.

$6_{5} 6$. i. Le Gavouć, Burf. VIII. p. 25 . Briss. Oruith. 0. Lisn. o.

2. Le Mitylene, Butf. VIII. p. 27. Briss. Ornith. o. Linn. o.

657. Coucou verd doré et blanc, Burf. XII. p. 42. Briss. Omith. O. Cut-
cullus caprius nihi Linv. Gell.

658. I. Serin de Provence, Burf. VII. 5. Briss. Otuith. III. p. 79. Fititgillu mantana, Linn. Gen. 112.37.

2. Venturon de Provence, Burf. VIII. p. 5. ferin d'Italic, Briss. Orwit ti. $^{2}$ III. p. 182. Fringilla Citrinella, Livn. Gen. I I 2. 16.

650. I. Grosbec tacheté du Cap. Buff. VI. p. 206. BRIss. Ominth. III. p. 171. pl. I. I. Fringilla Capenfis, Linn. 109. 39.

2. Grosbec d'Angola, Ruff. VI. p. 207. Briss. Ornith. o. Loxia Col laris mihi Liñ. Gen. 109. 0 .

660. 1. Varieté de la Rousfeline, Bufr. IX. p. 85. Briss. Ornith. III. o.
Alunda Linv. 105. 0.

2. Le Cujelier, BurF. IX. p. $3^{6 .}$ Alouette des Bois, Briss. Ornith. III. p. 340. Alanda arborea, Linn. Gen. 105. 3. 661. 1. La Rousfeline BufF. IX. p. 85. Alatida Briss. Ornith. o. LinN.
I05. O.

2. L.e Pipi, Burf. IX. p. 54.pl. IV. Alauda Sepiaria, Briss. Ornith. III. p. 347 . Alauda trivislis, Linn. Gen. 105. 5.

-662. La Coquillade, BufF. IX. p. 109. Briss. Ornith. III. o. Alauda matutinit, LINn. Gen. 105. 0.

66.3. Le plus grand Martin pecheur, Buff. XIII. p. 255 . Ispida Briss, Orzuth. o. Alcedo gigas mihi LiNn. 0. great brown hingsfisher, LATH.
tirds 1. p. 609. 1. 
Pl.

664. I. Ortolan a ventre Taune, Burf. VIII. p. 34. Hortukanus Be III. o. Emberiza flav.

2. Sa Femelle.

665. I. Varieti du moineau de Senegal, Burr. VI. p. 222 :

2. Sa Fenclle, voyez pl. 223. I.

666. I. Pctit azur, Buff. VIlT. p. 329. Mitfciapa Briss. Ornith. O. Mil fcicapa azurea, mihi Linn. Gen. 11 3. o.

2. Gobemouche Citrin, Burf. VIII. p. 336. Briss. Omit/. III. a: Mufcicapa Citrma, Lisw. Gen. 113 . 0 .

66\%. Lpeiche de Nubic, Burr. XIII. p. 28. Pic Briss. Ornith. III. c. Picus Nubucus mihı Linn. o. Nubian woodpcelice Lath. birds I. p. 5-6. 23 .

668. I. Bec figue, Buff. IX. p. 27=. Ficedula Briss. Omith. III. p. $3^{6}$. Motacilla ficedula, Linv. Gen. I I 4. I 0.

2. Fauvette des Alpes, Burf. IX. p. 226. pl. X. Briss. Orint'i. IIL. O. Linn. Ger. 114 . o.

569. 1. Varicté du Pirpit bleu, Burfon X. 25.

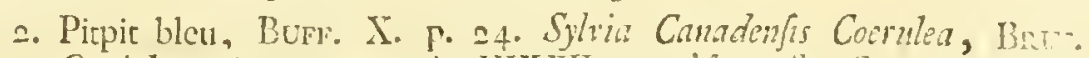
Ornith. IfI. p. 534. pl. Mivill. 1. Motacilla Cayanat, Lixv. II 4.40.

3. Pitpit varié, Bufr. X. p. 27. Sylvia Surinamenfis Catrulea, Briss. Omili. III. p. 536. Motacilla relia, Linn. Ger. I1 4. 48.

67o. 1. Souimanga dore changeant, a longuc quete, Burr. X. p. 278 . Grimpercau a longue queue cu Senegal, Briss. Omith. III. p 6+5. Cer. thicu fulcella, Linn. Gen. 65. 19. Beautiful Crecper, Latri, buit's I. p. 719.20 .

2. Soumanga a Capuchon violet, BerF. X. p. 276. 1. Grimperenu a long'te queue du Cap de bonne Esperanee, Bruss. Ommth. IH. p. 649. pl. XXXII. fig. 6. Certhin violacea, Linv. Gen. 65. 22. violet hoaded Creeper, LaTri. lirds I. p. 7I8. n. I 9.

671. 1. Colibri a cravatte verte, Burr. X\%. p. 7. Mellifuga Brts. On mith. III. o. Trochilus wiridgulat, Lins. Gen. 65. 0. green throatsd Humming bird, Larru. hirds I. P. 755. 14.

2. Colibri a queue violetre, Burw. XI. p. ¡6. Mellifega Briss. Omith. o. Trochilus violicautu, Lunv. Gen. 66. o. violet taled Hemmingbiri Lat11. birds I. p. 75 + I I.

672. 1. Le Colibri amethylte, Burron. XI. p. 20. Briss. Omith. III. o.

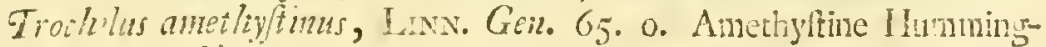
bird LaT1. birds I. p. 787. 17..59.

3. Cravatte doréa, Burr. XI. p. 33. Mellifuga Ciyanenfis yentre allo Briss. Ornith. IHI. p. y07. Trochilus mathyo, Linn. Gen. 66. 10. gold Throated Hummingbird, Lath. birds I. p. 77. 43.

2. Oifenu Mouche a lareses tuvan, Bufr. XI. p. 8. Briss. Omith. c. Lisn. 66. o. Trochilus largipennis mihi. Broad thafed hummingbird, LATH. hirais I. 1. $7 \sigma_{5}$ 3०.

673. Mnrtin pecheur a Coife noire, Burron. XIII. p. 278 . Ispida Briss. Ornith. o. Alcedo fileata mihi LinN. 62. o. Black kapped Kingfișher, Lath. birds I. p. 624. 15 .

674. 1. La Bergeronnette grife, Buff. XI. p. 37\%. Briss. Ornith. III. p. 465. Motacilla cinerea, Linv. Gen. II f. O. 
$\Gamma 1$.

2. Bergeronnette du Printems, BufF. IX.p. $3^{8}+$. Bergeronnetre Jaune Briss. Omith. III. p. 468. Motailla flava, Linn. Gen. it 4 . 12.

675 . 1. Le Gillet, Bufr. VIII. p. 342. Mufcicapa Briss. Omitiz. III. a Linn. Gell. II 3. o. Mufcicapa Pica.

2. Le Rubin, Burf. VIII. p. 35 I. Briss. Omitho o. Mufcicapa restimus, Linn. 113.0.

6,6. 'Tyrar de la Caroline, Buff. VIII. p. 398. Briss. Ornith. o. Mufizcapa Carolinenfis, Linn. Gen. 113. o. Caroline Tyrant Chrike, LAn!. birds I. p. 186.37.

677. Noucherolle a queue fourchue, Burr. VIII. p. 376. Briss. Ornitio. Linn. Gen. II3. O.

6-8. 1. I.e Traquce, Buff. IX. p. 311. pl. XIII. 1. Ficedula miketra, Briss. Onathi. III. p. 4=8. Motacilla Rubicola, Lins. Gen. $11 \%$ 17.

2. Le Tarier, Bufr. IX. p. 323. Ficedula rubetra mijor, Briss. Oriuth. p. $43^{2}$. Motacilla iubetra, Linn. I I 4.16.

6-g. Martil pecheur huppé, Burr. XIII. p. 277. Briss. Omith. o. Linn. Gel. 62. 0. Alcedo guttate mihi. Great african Kingfisher, Latman lirds I. p. 6I I. 3. A. varieté du grand Martin pecheul de M. PALLAS Spic. VI.

680. Le plaftron blanc, Buff. XI. p. 84. Briss. Omith. III. p. 60. LenN. Gen. 66. grey necked Humming bird, Latis. birds ; $61 . \simeq 2$.

2. Sa Femelle.

3. Le Plaftron noir, Buff. XI. p. 82. Polytmus Famaicenfis, Briss. Ornith. III. p 679. pl. XXXV. I. Trochilus Mingo, Linn. Ger. 66. 10. Mango Humningbird, Latuan birds I. p. 758 . 19.

68 I. Grimpereau, Buff. X. p. 224. Certhia Brisson Onith. III. p. 603. Certhia familiaris, Linn. Gen. 65. I. Common Creeper, Latito Lirds I. p. 70 I. I.

2. Souimanga de l'The de Bourbon, Buff. X. p. 273 . Grimpcren, Briss. Ornith. III. o. Certhia Borbonica mihi. Linv. Geiz. 65. 0. yellow rumped Creeper, Lath. birds I. p. 736.4 \%

682. 1. Guirguit verd. Buff. X. p. 303. Certhia ziridis, Briss. Omih III. 0. All green Creeper, EDw. glean pl. 348. Lath. Girás I. p. 728. n. 28 .

2. Guitguit verd tachetć, Burf. X. p. 304. Grimpercau verd tacheté de Cilyenme, Briss. Onith. III. p. 636. pl. XNIII. 2. Certhia Cayma, Linn. Gen. 65. 9. Cayenne Creeper, Latio burds I. p. 728. n. 29.

68 3. T.c grand Lory, Burf. XI. p. 189. Briss. Ornith. IV. o. Pfittaclis (Larius) Ceclanenfis mihi. Linn. Gen. 45. o. Purperroode Loeri Voss. monogr. Grand Lory Latr. birds I. p. 275 . n. 81.

684. Lory rouge et violet, Burf. XI. p. 182. Briss. Ormith. IV. o. Pfittacus Squamatus mihi LinN. 45. 0. Gueby Lory, Lathan birds I. p. 219. 23 .

685 4.Figuicr olive, Burf. IX. Po 464. Briss. Ormith. o. Linn. Geis. II 4 . O. 
2. Figuier blen, Burf. IS. p. 446. Ficedula Canadenfis cinerea, Uriss. Ormith. ilI. p. 527. pl. XXVII. 6. Motucilla Canaden/is, Lines. Gen. 11+4.

636. 1. Con Jame, Burf. IX. p. 239. Ficedila Briss. Ornith. III. o. Mo. tacillu fuperciliofa, Linn. Gen. I 1 1. o.

68-. Oifenu Cendré (Manakin), Buff. VIt, p. 177. Briss. Ornith. o. Pitrol cincreal mihis. Linn. Gen. 115.0.

693. 1. Tamatia noir et blanc, Burr. Xill. p. 148. Barbu Briss. Ormith. III. o. Bulco Linn. Gen. 56. o. Luico leucomelas mihi. Lathani lirds I. p. 502 .

2. Barbi a plaftron noir, BuFf. XIli. p. 155. Barbu Briss. Ormith. O. Buico teitus mihi. Linn. Gen. 56. o. Black throated Barbet Lath. birds I. p. 507. 8. A.

689. Varieté du Tamatia noir et blanc, (688. 1.) grenter Pied Barbet Latrr. 1. p. 49 S.

690. Grand Pic noir a bec blanc, Burf. XIII. p. 69. Pic huppé de la Caroline, Briss. Oruith. IV. p. 26. Picus principulis, LinN. 59. 2. IVithe billed woodpecker, Laтн. birds I. p. 553. 2.

69r. I.e Palalaca, Lufr. XIII. p. 29. Pic Orangé, Briss. Oinith. II. o. Picus auruntius, Linn. Gen. 59. o. Bengal woodpecker Lath. Birds I. p. $58 \mathrm{I} .26$. B.

692. Epeiche rayé de la Louifanic, Buff. XIII. p. I08. Pic rayé Briss. Orwith. IV. o. Picus zebra mihi. Linn. Gen. 59. o. Caroline woodpecker, Latio. lirds I. p. 571. n. 17. B. varieté de Pic rayé de la Jaraique, $\mathrm{pl} .597$.

693. Pic a ailes dorées, Buff. XIII. p. 59. Pic rayé de Canada, Briss. Omith. IV. P. 72. Picus auratins, LinN. 59 9. the gold winged woodpecker, Catesb. Caral. I. 13. Latil. birds I. p. 597. 49.

69\%. I. Pic roux, Beff. XIII. 55. Briss Ornith. IV. o. Picus rufus; Livis. Gen. 59 o. rufous woodpecler, Latil. birds I. p. 595. 11. +5 .

2. Petit Pic noir, Burf. XILI. p. 81. Briss. Ormith. IV. o. Picus cruentatus, Miti. Lins. Gen. 59. o.

65. Pic verd de Pengale, Buff: XIII. p. 33. Picus Capitis bone Jpei, Briss. Omith. IV. p. 78 . the fpotted Indian woodpecker, EDw. bird's IV. p. I82. Picus Benghalenfis, Linn. Gen. 59. 13. Bengal woodpecler, Latii. lirds I. p. 580. n. 26.

606. Pic vert de Goa, Buff. XIII. p. 3 I. Briss. Ornith. IV. Linn, Gen. 59. o. Picus feflivus mihi. Goa woodpecker, Lath. birds I. p. 582. 11. 27.

697. La Huppe noire et blanche, BUfF. XII. p. 149. Briss. Ornith. IV. o. Upufa varill, Linn. Gen. 64. o. Madadagascar Hoopoe, Lath. birds 690. 2 .

698. Le Torcol, Burf. XIII. p. I25. Briss. Ornith. IV. p. 4. Yunx torquilla, LinN. Gon. 58. I. Latri. birds I. p. 548. 1. pl. 24.

699. Guerarou, Buff. V'III. p. 221 . Coinga gris Briss. Omith. II. p. 353. B. Ampelis grifea, LinN. I 08. O. varicty of grey thrike, Lath. Birds P. 183.36 . grey pie of Brafil, EDw. pl. 318.

700. 1. Palikour Buff. VIII. p. 238 . Briss. Ornith. o, Linv. o. Formicarius torquatur, mihi. 
Pl.

„. Carillonnetr Buff. VIII. p. 246. Briss. Ornith. o. Linn. o. Forinicarius Cantatar, milhi.

701. 1. Coraji Buff. VIII. p. 256 . Fourmillier Briss. Omith. o. Linv. o. Formicarius......

2. Alipi Burf. VIII. p. 257. Formillier Buss. Ornith. o. Formicarius attothorax, LinN. o.

go2. Roi des Fourmilliers, Buff. VIII. p. $=3 \mathrm{~s}$. Briss. Ornith. O. Lixx.

o. Formicarius varius.

703. 1. Colma, Buff. VIII. p. 24.1. Brass. Ornitit. o. Lans. o. Fomicarius Colmu.

2. Bambla, Bufr. VIII. p. 454 . Briss. Omith. o. Linn. o. Fornicarius Banlia.

70 1. I. Figuier gris de fer, Burf. IX. p. 454. Figuier Cendré de la Penfylvanie, Briss. Ormith. Sutpl. p. 107. Motacilla Coeralea, Lins. Gen. 154.43 .

2. Tiguier Protonotaire, Buff. IX. p. 465. Briss. Ornith. III. o. Motirilla citrea, Linv. Geir. 114 . 0.

7. I. Figuier bleu, Bufr. IX. p. 404. (la Femelle), Briss. Omith. III. o. Motacilla Sialis, L.ms. Gen. I I4. 38 ?

2. Petit Simon, Burf. IX. p. 407. Finguier de l'Ile de Bombon, Briss. Ornith. III. p. 5 10. pl. XXVII. 3. Motacilla Domarica, Livn. Gen. 114. 26 ?

3. Male du Figuier bleu, (n. 1.).

706. 1. Grand Befroi, Burr. VIII. p. 255. Bruss. Ornith. o. Formuarius brevicauda, Linn. o.

2. l'Arada, Bufr. VIII. p. 249. Briss. Ornith. o. Formicariuts Mufbcus mili. Lins. 0.

g07. 1. Plumet blanc (Manakin), BuFf. VIII. p. 175. Briss. Ornith. IIT. o. Pipra albifrons, Linv. Gen 115. 5?

2. Le Manikor, Bufr. VIII. p. 178. Briss. Ornit/z. o. Linv. Geil. 115.0.

708. I. Penduline (Mefange), Burf. X. p. 155. Briss. Oinith. İI. 1. i65. Parus pendulinus, Linn. Gen."116. 13.

2. Roitelet Mefange, Butr. X. p. 76. Calendula Briss. Omith. III. p. 579. Motacilla Regulus, Linn. I 4.48.

3. Mefunge a Ccinture blanche, Buff. X. p. 173. Briss. Ornith. o. Linv. Gen. 115. o. Parus cindus.

709. 1. Fauvette ombrée de la Lonifanic, Burf. IX. p. 234. Briss. Ornith. o. Linn. Gen. 114. 21. Motacilla coronatu. EDw. gleun 298 .

2. Faurette a poitrine Jaune de la Louifanie, Burf. IX. p. 235. Briss, Ornith. o. Motacilla chryfoptera, Lins. Gen. i 1s. 20. EDw. glean. 299.

710. Tangrivio, Buffon VII. p. 331. BRiss. Ornith. III. o. Lunn. Gen. III. 0.

71. 'Tangarou, Burron VII. p. 361. Briss. Ornith. III. o. Linn. Gei. I11. 0. Tamarara rufa. 
PI.

7 $\mathrm{I}$. Le Sirli (Alouette), Burf. IX. p. 91. Briss. Omith. III. o. Alaudie Caperzis, milhi. Linn. Gen. 105. 0.

73. Perroquet a bec couleur de fang, Bufr. XI. p. I69. Pfittacus fuscus, Briss. Ornith. IV. p. 314. Pfutarus megularyathos mihi. LinN. 45. O. grent billed Parrot, Latit. Biads I. p. 27 3. n. 86.

$\because$ s. I. Le gris olive (Tangara), Buff. VII. p. 392. Briss. Ornith. III. o. Linn. Ger. Ili. o. Tairagra grifer.

2. Tauggara Camail ou Cravatte, Bufr. VII. p. 356. Briss. Ornith. III. o. Linn. Gen. III. o.

715 , Martin pecheur ou l'Alcyon, Buff. XIII. p. 242. Ispida Briss. Or'mth. IV. p. 47 1. Alcedo Ispida, Lins. 62. 7. Common Kingfisfher, LATH. lirds I. p. 626. 16 .

?16. MIrtin pecheur Pic, BufF. XIII. p. 272. Ispida ex alto et nigro yaria, Briss. Ornith. IV. p. 520. Aicedo rudis, Lins. Gen. 62. ó. Black and Wiche Kingfisfher, Eow. 1. pl. 9. Latuan birds I. p. 612. 4 .

717. l'Ouantou, Burf. XIII. p. 272. Pic huppé noir de Cayenne, Briss. Ornith. IV. P. 3I. pl. I. fig. =. Picus lineatus, Linv. Geiz. 54. 4. lineated woodpeclicer, Latiun birds I. p. 556. 4.

7 I . Pic noir a huppe rouge, Dufr. XIII. p. 73. Picus virginianus criftatus, Briss. Ormth. IV. p. 29. Picuts pileatus, Linn. 59. 3. Larger redcrifted woodpecker Catesb. Carcl. I. pl. 17. pileated woodpecker; Latu. birds I. p. 559. 3०

719. Grand Pic rayé de Cayene, Bufr. XIII. p. 4 i Picus varius Americanus criftatus, Brisš. Ornith. IV. p. 3t. LiNn. Gen. 59. o.

720. 1. Tangara a gorge noire, BUFF. VII. P. 401. Briss. Ornith. III. o. Tamugra nigrigula, Likn. Gen. III.o.

2. La Coeffe noire (Tangara), Buft. VII. p. 401. Briss. Ornith. III. o. Tanagra fileata, Lins. III. o.

721. Le Secretaire, Butr. XIV. p. 3o. pl. IHI. Brisson Omith. o. Linn. o. fagittarius Vosa. monogr. fecretary, Latian I. p. 20. pl. 2.

722. IIrondelle blcue, Benr. XII. p. 442? Irindo freti Hudfonis, Briss. Oraith. app. 56? Hirundo fubis, Lisn. Gen. I $17.7 \cdot$

723. I. Hirondelle brune ex blanche a ceinture brune, BufF. XII. p. 450. Briss. Orwith. o. Hirundo Cindta mihi. Livn. 11700.

2. Hirondelle a Capuchon roux, Burr. XII. p. 350. BRiss. Ornith. o. Hirundo Cacullata mihi. Lins. Gerr. 1 i 7 . o.

724. I. Hirondelle a vertre roux, Buff. XII. p. 349. BRiss. Ornith. HII. o. Hirnndo erythrgafter mihi. Linn. Gen. II 1 . 0.

725. I. Petit martinet noir, Buff. XII. p. 433. Martinet de St. Domingo, Briss. Oraith. II. p. 5 I t. Linn. Gen. 117.0.

2. Martinet a collier blanc, Burr. XII. p. 438 . Briss. Ornith. o. Linn. Gen. $11 \%$ o.

726. 1. Hirondelle Cumarin, Buff. XII, p. 479. Briss. Ornith. o. Linn. 117.0.

2. Hirondelle acutipenne, Burf. XII. p. 477. Hirundo Carolinenfis, Briss. Onuith. II. p. 50 I. Hirundo Pelasgica, Linv. Gen. II 10. 
$\mathrm{Pl}$

72-. I.e Crigri (varieté du Toucan Amcari), Burf. XIIt. p. 189. Tucaud Catunenfis viridis, Briss. Ornitho IV. p. 4ะ3. pl. XXXIIL. 2. Ranifliafios Aracari, Linn. Gen. 46. 3. B. green Toucan, Lath. birds $3.3 \mathrm{I} \cdot \mathrm{I}$.

723. Sa Femelle:

729. La femelle du Coalic (Toucan) pl. 57\%.

7.jo. 1. Roitelet de Buenos Aires, Buffon X. 56. $\{$ varietez du Roitelet 2. Troglodyte de la Louifanic, Buffox ibid. $\{$ pl. 651.2.

73т. I. Jigguier cendré a collier, Buff. IX. p. 442. Briss. Ornith. o. Lins. Gitn. 114 . O. Motacillt eques.

2. Firtice de Misfisfippi je ne le trouve point cité chez M. Buffon。 732. Singoulevent (Tettecherre) acuti Penne, Bufr. XII. p. 26 f. Briss. Or mith. o. caprimulgus acutipents mihi. LinN. 110.0 .

733. Le MLontvoyaut, Buff. XII. p. 268. Briss. Ornith. o. Linn. Gen. 118. o. Cipprimulgus.

734. l'ibipu, Metr. Xil. p. 264. petit Engoulevent tacheté de Cayenne,

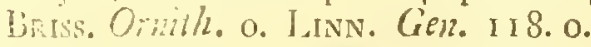

735. Engerlevent roux, Bufr. XiI. p. 268. Briss. Ornith. o. Linn. Gen. I 3 . O. Catprimulgas rufus, mihi.

¿36. Cournucou gris a longue Queue, Buff. XI. p. 402. Troson viridis

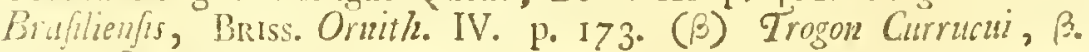
IINA. Gen 55. 2.

73-. Couroucou gris, Buffon, XI. p. 400 . Briss. Ornith. o. Linv. Gein. 55. o. Red bellied Currucu, Lathang birds I. p. 486. B.

738 . I. Alouette noire a dos fauve, Burf. IX. p. 33. Briss. Ornith. o. Linv. Gen. 105. 0. Alauda migra, mihi.

2. La variole, Burf. IX. p. 89. Bisss. Ornith. o: Lins: Gen. I05. O.

739. Foumicr, Buff. VIII. p. . . Briss. Ornith. o. Mlifcicupa, Linn. Geil. II 3. o.

740. Guepier verd a gorge bleue, Buff. XII. p. 196. Guepier a Collier de Aindugascar, Briss. Ornith. IV. P. 549. et 552. Mufficata viridis, Linv. Gen.63. 2 .

7+1. Thumgara du Misfisfippi, Burf. VII. p. 353. Briss. Ornith. o. Linn. Gen. 11 1. o. Tanagra Coccinea, mihi.

\% +. Oifeau Silencietux (Tangara), Buff. VII. p. 429. Briss. Ornith. o. Linn. Gen. I I I. o. Tanagra fllens, mihi.

74. Perruche varicé du Perruche a tete bleuc, pl. 61. Orange breafted Patirot, LaTh. birds I. p. 2 I2. I 4 .

744. Caica (Pertuche), Buff. XI. p. 394. Briss. Ornith. IV. o. Lins. Gen. +5.0 . Pflttacus Hiftrio', mihi. Hooded Parror, Lath. Birds I. P. 306. n. II 3 .

745. Cocy de Roche de Pcru, Buff. VIII. p. 187. varieré du Cocq de ro. che de la planche 797. Pipra rupicola, Linn. Gell. 115. I. (B).

746.1 . Tamatia, Bufr. XiIt. p. 139. pl. IV. Grivetre Briss. Omth. III. P. 213. Bucco Linn. Gen. 56. o. fpotted bellicd barbet, Lavife birds I. p. 494. 1. 
Pl.

\section{$(47)$}

2. Petit Barbu, Buff. XIII. p. 15\%. Briss. Omith. O. Linv. Ge 59. Bucco nanus, mili. black fpotted burbet LaTH. I. p. 496.2.

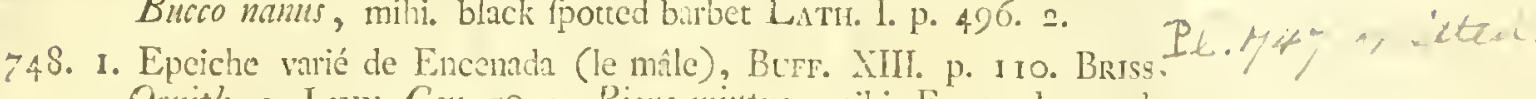
Ornith. o. Lunv. Gen. 59. o. Picus mixtus, mihi. Encenada wooupecker, LATH. birds I. p. $57 \% 2 \mathrm{x}$.

¿. Petie Epeiche brune des D.Iolucces, Buff. XIIr. p. roo. Briss. Ornith. o. Linn. Gen. 59. o. Brown woodpecker, Lam1. birds I. p. 577.11 .24 .

749. Ralle d'eau, Eufr. XV. p. 23\%. pl. XIII. Rallus aquaticus, BrIss. Oinitio V. p. 151. pl. XII. 2. Lrvis. Gen. 59. 2.

750. Raille de genet, BuFr. XV. p. 225. Oitygometra Briss. Ornith. V. p. 152. pl. XIII. fig. 2. Rallas Crex, LinN. Gen. 59. I.

751. Narouette, Buff. XV. p. ₹+2. Rale ftrié des Phalippines, Briss. Ornith. XV.p. 173. pl. XIV. 2. Ratlits ftriatus, Livn. Gell. 93. 5.

752. I. Fauvette tachetée de Ia Louifanie, Buff. IX. p. 233. 3. Ficedula Briss. Ornith. III. o. Linv. Gen. 114. O. Molacilla naevial.

2. Petire fauvette tacheréc du Cap. Bufr. IX. p. 233. 2. Briss. Ornith. III. o. Lunn. Gen. I f t. o. Motacilla muculo a mihi.

753 . Le Kiolo (Rale) le même que celui de la planche 368 . Burf. p. 233 . The american Water mill, Livw. glean p. I 44. pl. 279.

754. Epeiche velue de la Virginie, Burf. XIII. p. 11 1. P'icus sarius Virginuthus, Briss. Omitho IV. p. 48. Picus rillojuts, Linn. Gen. 59. 16.

755. Le IIcron Commun, Buff. XIV. p. 50. pl. V. Ardea Briss. Ornith. V. p. 39a. pl. XXXIV. P. 392. Aidea cineiea, Linv. Gen. S4. II.

Ђ氵6. 1. Vintfi Bufr. XII. 302. Ispida Indica crifiatu, Bress. Ornith IV. p. 506. Linn. Gen. 62. 4. (6). crutted Kingfisher, Latrio birds I. p. 632.17 .22 .

2. Intrin pechour verd Gange, BuFr. XIIJ. p. 321. Ispida Americanut rividis, Bruss Omith. IV. p. 400. Aliedo fupiriliofa, Lisv. Gen. 62. b. litele green and otange Kingfiner, EDw. glean 245 . fupercilious Kingfisher, Latr. birds I. P. $6+3 \cdot 34$.

3. Sa Femelle.

757. Nartin pecheur a tete et con, coulenr de Paille, Buff. XIII. p. 280. Priss. Omith. IV. o. Alcedo Favian, Linn. Gen. 62. 0. Withe headed Kingfisher, LaT11. birds 1. p. 617. 12. \&.

759. Le Bihoreau, Burr. XIV. p. 167. pl. VIII. Briss. Omith. V. p. 493. pl. XXXIX. Ardea Nyaftiorax, Liñ. Gen.84.9.

750. La Femeile.

760. Engoulevent varié, Burf. XII. p. 251. Caprimulgus Brtss. Ornith. o. Linn. Gen. I 18 . o.

76r. Btacneux, Burf. ... Frntercula Briss. Orwith. VI. o. Alea Cirratha Linn. Gen. 69. o. Pall. Ficil. zool. folsc: V.

762. Le Labbé a longue Quene, BuFF. XVI p. 249. Stercorarius longicantdus, Briss. Ornith. VI. p. 155. Larus parafiticus, Linv. Gelı. $7^{6 .}$ IO.

763. Petit Butor de Cayenne, BuFF. XIV. p. 179. Ardea ftellaris minor, I.INN. Gen. 84. 21. Ardoa Botamus, Briss. Ornith. 5. p. 444. pl. XXXVII. I. 
76 . Coucou tacheté de la Chine, Bufr. XII. p. 33. Briss. Ornith: IV.o. Citculus maculatus milhi. LinN. Cien. 57. o. Chinefe fpotted Cuckow, LaTH. birds I. p. 516.

755. Couroucou de la Gujane, BuFr. XI. p. 399. Couroucou cendré, Briss. Ornith. IV. p. 165. pl. XVI. I. 9rogon ftrigilatus, Lins. Gen. 55. 1. Cinereóus curucus Lath. bird's I. p. 489. n. 3 .

766. Phalarope cendré, Burr. XI. p. 3tб. Briss. Ornith. IV. p. 15. Tring lohata, Lins. Gen. 87. 8 .

$76 \%$ Perriche a front rouge, Buff. XI. p. 374. Briss. Ornith. IV. p. 3.39 : Pfittacus Canicularis, Linv. 45. 16. Red and blue headeci Parraliset, EDw. IV. pl. 176. Lath. livids p. 24ฯ. 40.

768. Perruche Souris, Buff. XI. p. 206. Briss. Ornith. IV. o. Pfittaius monachus, Linn. Gen. 45. o. gorcy breafted Parrakeet, Latis. I. p. 247.47 .

769. La Grue, Buff. XII. p. 41 S. Briss. Omith. p. 374. Ardea Crmis, Linn. Gen. 34.4.

770. Canard. Sarcelle de Madagascar (mate), Buffon. . . Briss Omith. VI. Linn. Gen. 67. 0. Anus autrita mihi.

771. Coucou brul piqueté, Burf. Xli. p. 31. Coucoul tacheté des Indes, Briss. Orinith. IV. p. 134. Cuculus punctatus, LinN. Gen. 57. 8. rufous fpotted Cickow, Latir. birds p. 5 i 7.8 .

772. Tacco Buff. XII. p. 65. Coucou a long bec de la Jamaique, Briss. Ornith. IV. p. 116. Cuculus vetula, LinN. Gen. 57. 4. long billed Rain Cuckow, Latir. birds I. p. 535. 11. 32.

773. Tiklin brun, Buff. XV. p. 248. Rallus Phitippenfis fursus, Briss. Ornith. V. p. 173. Rallus Philippenfis, Linn. Gen. 93. 4.

774. Tiklin Bufr. XV. p. 246. Rallus Philippen/is, BR1ss. Ornith. V. p. 163. pl. XIV. 2. Linn. Gen. 93. 5. Rullus firiatus.

775. Râle tacheté de Cayenne, Burf. XV. p. 255. Briss, Ornitho V. o. Linn. Gen. 93. O. Rallhs matulatus.

776. Canard fauvage, Buff. .... Briss. Ornith. VI. p. 3ig. Femelle du

777. Cunard. fauvage, Buff. . . Briss. Ornith. VI.p.3 3 8. Anas Bofchas, Linn. Gen. 6\%. 40.

778. I. Martin pecheur roux de Madagascar, Buff. XIII. p. 29.3. Irpiail Madagascarienfis, Briss. Ornith. IV. p. 508. pl. XXXVill. I. rufous IKingfisf her, LATis. birds I. p. 630. n. 19.

2. Martin pecheur roux, Burf. XIII. p. 294. Briss. Ornith. IV. o. LinN. Gen. 62. o. Alcedo rubra mihi.

779. Calao d'Abysfinic, Buff. XII. p. 230. Briss. Ornith. o. Linn. Gił。 47. o. Buceros abysfinicus Abysfinian Hornbill, Latı. hirds I. p. $34 \div \cdot 4$.

780. Calao de l'Ine de Panay, Burf. XIII. p. 215. Briss. Ornth o. Livv. 47. o. Buceros Panini, mihi. Panayan Hornbill, Latir. birds I. p. 353. 8.

78 r. La Femelle.

732. Caurale Buff. XV. p. 261. Briss. Ornith. 0. Linn. Gen. 86 o. Scolopax foluris, mihi. 
783 . I. Todier bleu a rentre Orangé, Buff. XIII. p. .37 . Bruss. Ornith. $\sigma$. Todis pittus mili. Linn. Gen. 61. 0. blue Tody, Lath. birds I. D. 659.4 .

2. Miartin pecheur a tete verte, BtrF. XIII. p. 279. Briss. Ornithoo. Linn. Gen 62. o. Alcedo Chloris, green headed Kingfis her, Lati. bir is I. p. 6ะ0.n. II.

784. Pctir Pic a gorge Jame, Burf. XIII. p. 56. Briss. Onith. o. Lins. 59. o. Picus flavigula, yellow hended woodpecker, Latham birds I. p. 595. n. 46.

785. Pic varié de la Caroline, Buff. XIII. p. I 15. Briss. Ornith. IV. p. 62. Picus \%urius, Linn. Gen. 59. 20. yellow bellied woodpecker, Latho lirds I. p. 574. n. $\approx 0$.

786. 1. Tres petir Pic de Cayenne, Buff. XIII. p. 57. Briss. Ornith. IV. p. 33. minute woodpecker, LıT11. birds I. p. 596. n. 48 .

2. Pic a tete grife, BuFf. XIII. p. 39. Briss. Oinith. IV. o. Picus grifeo cephalus, Linn. Ge1. 59. 0. Cape woodpecker, Latis. birds I. p. 586. n. 34 .

787. Le même que je Heron Commun, pl. 755.

788. Heron pourpre, BuFf. XIV. P. 90. Ardea purpurafcens, Briss. Ornith. V. p. 424. pl. XXXVI 2. Arata purpurea, LinN. Gen. 84. 10.

789. Le Butor, Buff. XIV. p. 150. pl. VII. Botaurus intss. Ornit/. V. p. 444. Aidea fellaris, Linv. Genı. 8\%. II.

790. I'Onoré Buff. XIV. p. 180. Briss. Ornití. V. p. 400. Ardea Cayanellfis criftatal, Linv. Gell. 8 4. 14 . Aidea Cocoi.

79r. I. Perruche a ailes variées, Buff. XT. p. 24 I. Briss. Ornith. IV. o. Linn. 45. o. Pfittaca Batarica mihi. Biacliwinged Parrakeet, Broune illifiral, Tab. 8. LATH. birds I. p. 316. n. I23.

2. Perrucine a tete Grife, Burf. XI. p. 240. Pfittacula Madagascarienfis, IRIss. Ornith. IV. p. 394. pl. XXX. 2. L.INN. Gen. 45. o. grey healed Parrakect, LATH. birds I. p. 315. n. 122.

792. Papegay a bandeau rouge, Buff. XI. p. 339. Briss. Ornith. IV. o. Linn. Gell. 45. 0. Pfittucus vittatus mihi. Red banded Parrot, LATr. brds I. p. 300. 11. I0.4.

793. Gurn-panga, Buff. VIII. p. 2 I I. Cotingu alba, Briss. Ornith. II. p, 356. Ampelis nivea, Linn. Geir. I08. 0.

794. La Femeile.

795. Le Courvite, Burf. XV. p. 199. Briss. Omith. o. Linn. Gen. o. Nouvean genre.

796. Ombrette, Buff. XIV. p. 192. Scops Briss. Omith. V. p. 303.Linv. Gen, o.

797. Grande Foulque a crête, Burf. XV. p. 349. Briss. Ornith. VI. p. o. Linn. Gen.91. o.

798. Canard a Collier de Terre neuve, Buff. . . B Briss. Ornith. VI. $\mathrm{p}$. 362. Anas hiffrionica, Linn. Gen. 67.35.

799. Femeile du Canard a Collier.

800. Pluvier Couronné, Burf. XV. p. 159. Briss. Ornith. o. Linn. Gen. 88. o. Charadrilts Coronatus mihi.

801. Pluvier a Aigrettes, Buff. XV. p. 156. Plurialis Senegallenfis armata, Briss. Ornitho V. p. 86. pl. VII. fig. 2. Charadrums Jpinofus, LinN. Gein. 88. 12. 
P1.

80z. Curard Garrot, Buff. ... Briss. Ornith. VI. p. 416. pl. XXVII. 2. Anins Clangula, Linn. Gell. 67.23.

803. Le millowin, BuFF... Briss. Ornith. VT. p. 384 . pl. XXXV. 1. Amas Perielope, Linn. Gen. 67. 27. voyez pl. 825.

80,. Canard fifeur de St. Domingo, Burf. . . Canard fineur de la Jamai. que, Briss. Ormith. VI. p. 403 . Anas arborea, Lins. Gen. 67.44.

\$๑5. Sarceile male de la Chine, Bufr. . . Q Querquedul a Chinenfis, Briss. Orimith. VI. p. 450. Aizas galericulata, Lins. Gen. 67. 42.

806. Femelle de la Sarcelle de la Chine.

807. Vanneau armé des Indes, Buff. "XV. p. ior. Briss. Ornith. Y. o. Tringa Indica, Lins. Gen. 17. 0.

808. Canard de Maragnan, Burf. . . Briss. Ornith. VI. o. Lisv. Gera. 67.0.

309. 1. Le organifte espece de Tungaras

2. Tangara nordoré, Buff. VIII. p. 358. Tangam fulva, Briss. Ornith. o. Tanagra, Liss. o.

810. La Poule Sultane, Buff. XV. p. 302. Porphyrio Eriss. Omitl. V. p. I22. pl. XLIl. Fulica Porplayrio, Linn. Gen. 91. 5.

811. Coucou, Burf. XI. p. 426. Briss. Ornith. III. p. 105. Cuculus Cat.. noris, Linn. Gen. 57. I. Common Cuckow, Latir. bira's I. p. 509. I.

812. Le Coucou brun varié de roux, Buff. XII. p. 77. Concou tacheté de Cayeme, Briss. Ornith. IV. p. 127. Cuculus survius, LiNs. Gen. 57. 9. - Ppotied Cuckow, Lath. birds I. p. 332. 38.

SI 3. Le petit viellard, Bufr. XII. p. 63. Coucou de la Jamaique, BRrss.

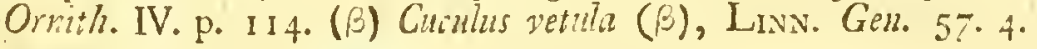

814. Petit Coucou de l'the Panay, Burf. XII. p. 37. yellow bcllied Cuckow, LATH. lirds I. p. 527. n. 22.

815. Coucou verdatre de Madagascar, Burf. XII. p. I 3. BRiss. Omith. IV. o. Cuculus gigas, Linn. Gen. 57. o. great Madagassar Cuckow, Lati. bira's I. p. 521. 11. 12.

816. Le veillard a ailes rousfes, BuFf. XII. p. 63. Concou de la Caroline, Briss. Ornith. IV. p. I 2. Cuculus Arkericunus, Linv. Gen. 57. 10. Caroline Cuckow, Lath. bivds I. p. 537. n. 35 .

8I7. Jabiru, Burf. XII. p. 408. Cicogne de la Guijase, Briss. Ornith. V. p. 373. Myateria Americama, Linn. Gell. 82. 1.

318. Courli, Buff. XV. p. 28. Numenins, Briss. Ornith. V. p. 3 I r. Scolopux arquata, Linn. Gen. 86. 3 .

319. Courli vert, Buff. XV. p. 44. Numerius risidis, Briss. Orkith. V. p. $3 \simeq 6$.

820. Courli des Bois, Buff. XV. p. 44. Briss. Ornith. V. p. 85. pl. XXVII. fig. 2. Tumtalus Fulcinellus, Linv. Gen. 85. 2:

821. Tetema de Cayenne, Buff. VIII. p. 242. Briss. Omith. o. Linn. Gell. o. Fomicorius Cayanenjis.

822. 1. Fourmillicr a oreilles blanches, Buff. VIII. p. 245 . Briss. Omith. o. LinN. 0 .

2. Sa Femelle.

823. 1. Fourmilier Befroi, Burf. VIiI. p. 23.7.

2. Fourmillier Je ne he trouve point dans l'ouvrage de M. Burfor. 
Pl.

824. Coucou des Philippines variete de 295 .

825. Cunard Sifficur, EUrF. ... Anas filtuluris, Briss. Ornith. VI. p. 39 r. pl. 55.2. Anas perielope, Linn. Gen1. 67. 27.

826. Cinnard Sintur de Caycune, Burf. . . Briss. Ornith. VI. p. 400 . pl. XXVII. I. Anas autumalis, Lisn. Gen. 67.

827. Chevalier rayć, Burf. XIV. p. 304. Totamus friatus, Bruss. Ornith. V. p. I96. pl. XVIII. I. Thinga Jiviata, Linv. Gen. 87.5 .

828. Tinamou varié, Buff. VhI. p. 294. Bniss. Ornitl. II. o. perdrix Tetrao Livin. Gekl. 103. 0.

829. Soni on petit Tinamou, Burr. VIII. p. 296. Briss. Ornit/. II. o. Tetrao pileatus, Linn. Gen. 503. o.

830. 1. Barbichon de Cayenne, Burf. VIII. p. 330. Mufcicaya Briss. Oin!th. O. Linn. o.

2. Sa Femelle.

$\$_{31}$. 1. Gobenotiche roux a poitrine Onangée, Burr. VIII. p. $3^{S} 5$. BRISs. Ormith.o. Linn. Gein. 1.13. Mutcicapal eques.

2. Petit Gobemouche tacheté, Je ne le trouve pas cité chez M. Buffon!

832. Pluvier Guighard, Buff XV.p. 1.37. Pluvialis Morinellus, Briss. Ormith. V. p. 54. Charadivis morinellus, Lisn. Gen. 88. 5.

833. Plivier armé de Cajenne, Burf. XV. p. 16 r. Brıss. Ornith. V. o. LiNv, o.

837. Pluvier Coiffé, Buff. XV. p. 158. Briss. Ornith. V. o. Linn. Gen。 88. o. Cizaradíns tecius, mihi.

835. Vanneau armé de la Louifunie, BuFF. XV.p. 103. Vanellus Ludoriciamus arnatus, Briss. Ormitio. V. p. I I5. LiNn. Gen. 87. 0. Tringa miles.

836. Vanneau armé de Cayenne, Bufr. XV. p. I05. Bruss. Ornith. V. o. Linn. Geil. o.

837. Tirica ou petite Jaseufe, Betr. XI. p. 393. Pfistacula Braflienfis, Briss. Ornith. IV. p. -38s. Pfitcacus Tiriacula, mibi. Linn. Gcn. 45o. grreen Parralicet, Lathas birds I. p. 320. 11. 129.

$8_{3}$ S. Le Perroque fuperbe, Je ne le trouve point cité chez M. Buffon.

839. Lo Crik, Burr. XI. p. 321: Pfittacus Cajanenfes, Briss. Ornith. IV. p. 337. P/ittacus agilis, Linv. Geit. 45. 20. Agile Parrot, Latri. birds I. p. 29?. n. Is.

8fo: Le Tavoua (Papegay), Bufr. XI. p. 33. Pfittacus Cyznocephalus Gujanenfis, Briss. Omith. WV. p. 247. Prattucus feftutus, Linv. Gen. 45. 35. Fellive Parrot, Latir. birds I. p. 298. n. Io?.

841. Le Courlis huppé, Bufr. XV. p. 5 t. Briss. Onuth. o. Linn. Gen. 85. 0. Tantalus crifatus mihi.

8q2. Petit Courlis, Burr. XV. p. 4 r. pl. III. Numenius minor, Briss. Or nith. V. p. 317 . Siolop 2x Pliveopus, Linn. Gen. 86. 4.

8+3. Beccasfeau, Burf. XIV. p. 329. Culblanc Briss. Ornith. V. p. 17\%. Tringa glareola ( $\beta$ ) Linn. Gen. 87. 13 .

8+4. Chevalier Commun, Burr. XIV. p. 296. Totamus Briss. Ornith. V. p. 188. pl. XVII. 1. Siolopax Calidris, Linn. Gen. 86. 1 I.

\$45. Chevalier a pieds rouges, Burf. XIV. p. 300. pl. XIV. Fotanius-ruber Briss. Ornith. Y. p. ign. pl, XXIll. 1. Siolotax totihus, Linv. G'n. S6. 12. 
乌ì.

3+6. Macama varié, Butr. XV. p. 300 . le Chirurgien varić, Briss. Ornith. V. P. I 29. Parra variablis, Liñ. Gen. 92. 4.

8.T. Perit Rale de Cayenne, BuFr. XV. p. 259 . Eriss. Ornith. V. o.Lins.

- Gen. 93. o. Rallas flasirevater mibi.

348. Le Comlan, Buff. XIV. p. 194. Briss. Ornith. V. Linn. Gen 85.0. 849. Rale a longec, Burf. XV. p. 25 I. Briss. Oimith. V. o. Linn. Gen. 93. o. Ralius longirofiris.

850. La Guignette, Buff. XiV. p. 338. Briss. Omith. V. p. I83. pl. XVI. 2. Tringa liypoleucos, Livin. Ge'm. 87. I 4.

851. Alouette de Mer, Mugf. XIV. p. 352. Briss. Onith. V. p. 2i . pl. XIX. I. Tringa Cinclus, Linn. Gen. 87. I 3.

852. Le Cincle, Butr. XIV. P. 358. Cinclus torquatus, Disss. Omith. V. p. 2i6. pl. XIX. fig. 2. Tringa alpina, Linn. Gen. 87. II.

\$53. Vanneau fuisfe, Buff. XV. p. 95. Baiss. Úmith. V. p. 207. pl. X. I. Tringa Helvetlat, Linn. Geiz. 87. I 2.

854. Vanneau Pluvier, Burs. XV.p. 107. Vanellus grifeus, Briss. Ornith. V. p. 100. pl. IX. I. Tringa Squatarola, Linv. Gen. 07.23.

855. Oye Bernache, Burf. ... Briss. Omith. VI. p. 300. Anus Bernicla, Linv. Gell. 67.13.

856. Le Tourne picre, Bufr. XV. p. 201 . pl. . . le Coulon chaud, Briss. Orinith. V. p. 132. Tringa interpres, Linn. 87.4.

857. Varieté du Tourne pierre.

858. Heron brun, Burf. XIV. p. 108. Heron gris Briss. Omith. V. p. 41=? Ardea grifea? Linn. 84. :2.

859. Ieron Agami, Bufr. XIV. Cancrophagus coernleus, BRIss. Ornith. V. p. 484. Ardea Coerulea, Linn. Gen. 84. 17.

860. Onowé rayé, Bufr. XIV. p. 18 1. Briss. Ornith. V. 0. Linn. Gen.84. o. Ardea lineata, mihi.

861. Cric meunicr, Buff. XI. p. 3I6. Briss. Ormith. IV. o. Linn. Gen. 45. o. Pfittaus furinofus, Mcally green Parrot, Lath. birds I. p. 290. 11.94 .

362. Perroquet verd a tete bleue, Burr. XI. p. 170. Briss. Ornith. IV: o. Linn. Genl. 45. o. Amboina Parrot, Lath. birds 1. p. 279. 37.

863. Pic a Cravatte noire, Buff. XIII. p. 53. Briss. Ornith. II I. o.Linn. Gen. 59. o. Plizus torquatus mili.

864. Perruche Ara, Buff. XI. p. 337. Briss. Omith. IV. o. Lins. Gen. 150. Heittacus manilatus milhi. L'arrot Maccaw. Lath. birds I. p. $=05.5$.

865. Grue a Collicr, Bufr. XIV. p. 446. Briss. Omith. V. O. Linv. Gen. 8. o. Grus Colluris mihi.

866. Cicogne, Burf. XII. p. 370. Briss. Omith. V. p. 365. pl. XXXII. Airdea Ciconia Linn. Gen. 8 +. 7 .

86\%. Courlis a tete nue, Burf. XV. p. 48. Briss. Omith. V. o. Linv, Ger. 55. o. Tantalus Cabutts mihi.

863. L. Couricaca, Burf. XiII. p. 403. Numemius Americants major, Briss. Ornith. V. P. 335. Tantalus loculator, LinN. Gens 85. I.

869. Sivacou, Burf. XIV. p. 195. Cochlearius fuschs, Briss. Omith. V.

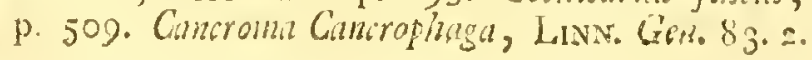


870. Bubu verd, Beff. XIII. p. I6I. Briss. Omith. III. o. Linn. Gen. 56. O. Bucio viridis, green barber, Lath. birds I. p. 504. I. voyez L.ATH. I. ¿ pl. 22 .

87. Le grand Barbu, Buff. XIit. p. 159. Briss. Omith. III. o. LinN. Gen. 56. o. Ducco virens, grand Barbet, Lath. birds 1. p. 503. Iо.

872. Jacobin huppé, Buff. NII, p. 35. Briss. Omith. IV. o. Linn. Gen. 5\%. o. Cuculus Jacobinus.

873. Calno de Malabrr, Bufr. XII p. 22 i. Briss. Omith. IV. Linn. Gen. 47. o. Buceros Coronutals, mihi. Fied I Iornbill. Latit. birds I. p. 349. pl. XI. Low. 231 . D. In figure de Larn. fimble une varieté, ou difference de fixe.

874. Barge Commume, Bufr. XIV. p. 278. Limo Ja, Briss. Ornith. V. p. 276. Siolopux Limofar, Liñ. Ċen. 86. I 3 .

875. Barge brune, Burr. XIV. p. 291. Linnofa fusca, Briss Ornith. V. p. 276. Scolopax fusca mihi. Linn. Gen. 36. o.

8 7 6. Barge aboyeufe, Burf. XIV. p. zSo. Linofat grifea, Briss. Oruith. V. p. 267 pl. XXIIr. 1. Scolopax Totanus, Linv. Gen. 86. 12.

87\%. La Poule d'Eau, Burf. XV. p. $26 \hat{3}$. Gallinula Briss. Ornith. VI. p. 3. pl. I. fig. I. 2. Fulica Chloropus, Linv. Gen. 9 1. 6. 4

878. I'Echasle, Burf. XV. p. 177. Huemantopus, Briss. Ornith. V. p. 33. pl. III. I. Charadrius Hatemantopis, Linn. Ge». 88.11.

879. Le Pic verd rayé voyez le jcune planche, 37 I.

SSo. Pluvier a lambcaux, Buff. XV. p. 160 . Briss. Ornith. V. o. Linn. o. Charadrius mulaburitus mihi.

88ュ. Becastine de la Chine, Bupr. XIV. p. i7ะ. Gallinago Madrespatana, Briss. Ornith. V. p. 308. Linn. Gen. 86. o. Scolopax Chinenfis, milhi.

SSz. Perdhix de NTer, Buff. XIV, p. 312. Glutreola Briss. Ornith. V. p. I 4 I. pl. XII. I. Hurudo Prutincola Linv. Gen. I I 7 . I2.

$38_{3}$. La Beccasfine, Burf. XIV.p. 252. Gallinago Briss, Oruith. V. p. 298. Scolopax Gallinago, Linn. Gen. 86.7.

83 4. Petite Beccasfine, Bufr. XIV. p. 263. Gallinago minor, Briss Ornith. V.p. 3०+. pl. X.VVI. 2. Scolopax Gallinala, Linn. Gen. \$6. 8.

885. Bccasfe, Burf. XIV. p. 223. pl. XI. Scolopax Briss. Ornith. V. p. 292. Siolopax rufticoli, Linv. Gen. 86. 6.

836. Heron blanc, Burf. XIV. p. 83. Ardea Candida, Briss. Omith. V. p. 4.28. Aidea alla, Linn. Gen. 84. 24 .

857. Grande Perriche a longs brins, Buff. XI. p. 2I7. Briss. Oinith. IV. o. Pfittacus longacalidul, Linv. Ger. 45. o. Malacca Parrakeet, Larir. lirds p. 241. 1. 39. C.

388. Perite Perruche a rete Couleur de rofe; a longs brins, Burf. XI. p. 215. Pfitucial Bengalenfis, Briss. Oimith. IV. p. 348. Pjittaicus rofa mihi. Linn. Gen. 45. o. Rofe headed ring Parmalicer, Evw. glean. 233. Latir. birds I. p. 239. n. 39. A.

889. Gue blanche, Bufr. XIV. I. Grus Americana, Briss. Ornith. V. P. 382. Aidea Americana, Lann. 84.5.

890. Calia Toc. Bufr. XiII. p, 2 г o. Lytrocorax fenegaienfis melanoryuchus BRiss. Ornith. IV. p. 573. pl. XLVT. I. Buceros Nafutus, Linv. Gern. 47. 4. le même que celui de la Planche 26ra. mais plus agé Black billed IIornbill. Lath. birds I. p. 35t. 10. 
11.

891. Calao des Manilles, Burf. XIII. p. 21 g. Briss. Omith. IV. o. Linn. Gen. 47. o. Buceros Mubill. Manilla Hornbill, LaTn. birás I. p. $354 \cdot 9 \cdot$

892. Le Courvite, Buffon XV. p. 199. genre nouveau approchant du Pluvier.

893. Grebe faulque, Burf. XV. p. 385. Briss. Oinith. o. Linn. Gen. 75. O. Colyntus fulica mihi.

894. Natuitui (Marcin pecheur), Ispida brafilienhis nevia, Briss. Ornith. IV. p. 524. Aícedo fusca mihi. Linn. Gen. 62. o.

895. Becasfe des Sivanes, Buff. XIV. p. 249. Briss. Ornith. V. o. Scolopax undulatu mihi. Livin. Gen.36. o.

\$96. Poule fuitane brune, Burf. XV. p. 3is. Porphyrionis yarietas Briss. Ormith. V. o. Fullia chmenfis mihi. Lins. Gen. gr. o.

897. La Favorite, Butr. XV. p. 323. Briss. Omith. V. p. 526. Linn. Gen. 91. Fulica parya, celle ci elt peue cerre la femelle.

898. Petir Crabicr, Burr. XIV. p. 126. Camorofagus Plitippenfer, Briss. Omith. V. p. 474. Linn. Gen. 34. o. Ardea pumila mili.

892. Bihorenu de Cayenne, Buff. XIV. P. I91. Briss. Omith. V. o. Linv. Gen: 84. o. Lirder Nyticorax Cayanenfis.

200. Barge rousfe, Buff. XIV. p. 28 +. Limofa rufa Briss. Omith. V. p. 28I. pl. XXVI. Scolopux lapponica, Linv. Gen. 86. 15.

901. Aigrette (Heron), BuFr. XIV. p. 95. pl. VI. Egretta Briss. Ornitho

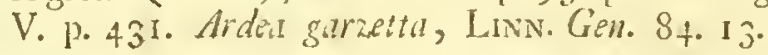

902. Aigrette rousfe, Bufr. XIV.p. I03. Briss. Omith. V. o. Linv. Gen. 84. o. Ardea ralfin mihi.

903. Guiltemot, BufF.... Uria Briss. Omith. VI. p. 70. Colymbus Truile, Iann. Gen. $75 \cdot 2$.

904. Pluvier dori , Bevf. XV. p. 12- pl. V. Pluvialis aurea, Briss. Ormili. V. P. 43. Challodruts plutiatis, Linn. Gen. 88.7.

905. Caftagneux, Bur. XV. p. 3-9. pl. XX. Grebe de Rivicre, Briss. Ormith. VI. p. 59. Colymtus stuithis, Linn. Gen. 75. 8?

906. Heron violet, Burf. XIV. p. 92. Briss. Omath. V. o. Linv. Gell. $8_{4}$. o. Arda Epissopus mihi.

207. Heron blanc a Calote noire, Burf. XIV. p. 107. Br.1ss. Cmith. V. o. Linn. Gen. 84. o. Aidel pileata mihi.

908. Cmbier gris, Bumt. XiV. p. 147. Briss. Ornith. V. o. Linn. Gen. 84. o. Cuncroma grifer, milhi.

909. Crabier roux a tête et qucue vertes, Burf. XTY. p. J f6. Triss. Or. with. V. o. Linv. Gen. 84. o. Aidea Chleropatura mibi.

910. Crabier de Coromaniel, Buff XIV. p. I 2 . Brass. Orinth. V. o Cancroma Coromanda mihi. Lisn. Gen. 83. o.

911. Cubier binne et brun, Burf. XIV. p. 125. Briss. Omith. V. o. Cuncromu learcopter an mihi. Lins. Gen. 84. 0 .

912. Crabier verd tacheté, Buff. XIV. p. 143 . Briss. Ornith. V. o Cuncro. ma maildat milni, Linn. Gen. 8 3. 0.

913. Cygne, Burr... Bruss. Ornith. VI. p. 292. pl. XXvill. Linn, Gers. 67. 1. Stizus Cygmus.

914. Le grand Plongeon, Buff. XV. p. 390. Mergas miljor, Eriss. Or-

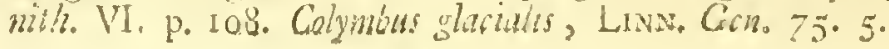


P1.

915. Le Courlis blane, Butr. XV. p. 62. Numenius Braftienfss candidus, Briss. Omith. V. p. 339. Scolopax alba, Linn. Gein. 86. 17.

916. Grande Barge rouge, Eurf. XIV. p. 266. Limoja rufa major, Briss. Ormth. V. p. 284. Scolopax aegocephalu, Linv. 86. I6.

917. Petit Guillemot (femcile), Burr... Uria minor, Briss. Omith. VT. p. 73. Allow Alle Linn. Gen. 69. 5.

918. Le Pluvian, Burf. XV.p. 163. Buss. Omith. V. o. Linn。Gen. 88. o. Churadruis niger.

919. Courlis de Terre, Buwr. XV. p. I 64. pl. VII. grand Pluvier, Briss. Ormti. V. p. -G. Cindiadruis oedicnemas, LivN. Gen. 38.10.

220. Pluvier a Collier, BuFf. XV. P. 142. Pluvialis torquata minor, Briss. Oinith. V. p. 62. pl. V. fig. 2. Chatratrius liatucald, Lisn. Gene 88. 1.

927. Le niême peti.

922. Beccasfine de Madagascar, Buff. XIV. p. 27r. Numenius Madugasa carrienfis, Briss. Ornith. V. p. 321. pl. XXVIII. Scolopux Madagascarienfis, Linn. Gerr. 86. 2.

923. Vanneau armé, Buff. XV. p. i I2. Briss. Ornith. V. p. 103. Tringa varia, Linn. 87. $2 \mathrm{I}$.

924. La Guiferte (Hirondelle de Mer), Buff..XVI. p. 87. Hirondelle de Mer tachetée, Brtss. Ornith. VI. p. a IG. pl. XX. fig. 2. Sterna nac yil, Linv. Gen. $77 \cdot 5$.

925. La grande Aigretre, Burf. XIV. p. ior. Briss. Orinth. VI. o. Linv. Gej. 8+. o. Aidea Garzette milor mihi.

926. Crabier noir, Buff. NIV. p. I 2 G. Briss. Ornith. V. O. Linn. Gen. 8.

927. I.e Cormoran, Buff. XVI. p. 43. Plaalacrocorax Briss. Ornith. VI. 1.511. Peliomus Carbo, LinN. Gen. 22. 3.

928. Canard, Buff. . . Canard fiflent huppé, Briss. Ornith. VI. p. 400.

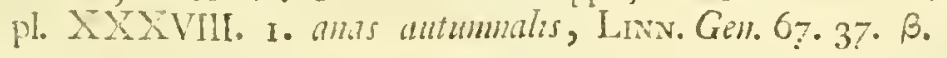

929. Iluicrier ou pic de Mer, Buff. XV. p. 185. pl. 1X. oftralegra Briss. Ormtit. V. p. $3^{8}$. pl. [iI. fij. 2. Hematopus oftralega, LiNn. Gen. 90. 1.

930. Canard, Burf. ... Briss. Omith. VI. o. Linv. Gen. 67. O. Anas

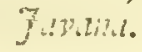

931. Le jongris, Bufr. XV. p. 314. Briss. Ornith. o. Colymbus grifegena, mihi. Liviv. o.

232. Le Bec ouvert, Burf. Xiv. p. 147. Briss. Omith. o. Linn. Gen. 8\%. o. Ardea ofitans mihi.

933. Calao a Casque ronk, Buff. XII. p. 236 . Betss. Ornitle. IV. o. LInn.

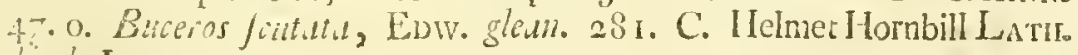
birds I. p. $3 \div 3 \cdot 2$.

934. Calso Rhinoceros, Bufr. XII. p. 208. Hydrocorux Indicus, Briss. Ormith. IV. p. 57 I. Buteros Rhinoceros, Linn. Gen. 47. 3. EDw. gleun. 281 . B. le bec. Latil birds I. p. 342. 2.

935. Harle Couronné, Bufr. XV. p. 437. Merganer Virginianias Crifta1us, Briss. Oinitis. VI. p. 25ü. Mergus chithlutus, Lann. Geno 63. I.

336. Sa Fenelle. 
P1.

937. Oje de la cote de Coromandel, Burf. . . Briss. Ornith. V1. o. Linv. Ger. 67. 0. Ánas tricolor mihi.

938. Girenier, Buffon. XII. p. 173. Briss. Omilh. V. p. 532. Mesops apiafier, Lins. Gen. 63. 1.

939. Poncre, Burfon. XIll. p. 1?3. Botaurus mavius, Briss. Ornith. V. p. 462. Aider mitrit, LinN. Gen. 84. o.

940. Merle dieau, Butr. p. 207. Briss. Omith. V. p. 262. Stumus Cinclus, Linn. Ge\%. 106. 5 .

94I. Le Grỉbe, Burf. XV. p. 30I. Briss. Omith. VI. p. 34. Colymbess Urinator, Liwn. 75. 9?

942. Varieté du même, (pectic Grêbc).

943. Grêbe de la Louilanie, Burf. XY. p. 373. Briss. Omith. o. Colymhus Ludoricianus, mihi. Livis. Gen. 75 . o.

944. Grêbe huppé, Burf. XV. p. 361. Colymlus criftatus Briss. Omint. VI. p. 38. Linn. Gen. 71. o.

945. Caftagneux des Philippines, Buff. XV. p. 38 I. Briss. Omith. VI. p. 59. Linn. Gen. 71. 8.9. Colymbus aurtus?

946. Canard Sarcelle, Buff. . . Briss. Ornith. VI. p. 329. pl. XXXII. i. Amas clypeali, Linn. Gen. 67. 19. (E).

947. Canard Sircelle, Bumf. . Pecire Sarcelle, Briss, Omith. VI. p. 436. pl. XL. I. Amls Gresca, Linn. Gen. 67. 33 .

948. Camard Sarcelie de la Louifanie, Burr. . . . . BRrss. Ornith. VI. p. 461.pl. XLI. fig. 1. Anas albeoh, Lisv. Gen. 67. 11. I8.

949. Canard, Bufr. .

950. Sa Femelle.

951. Harle, Burf. XY p. 414. pl. XXIII. Morg.mer Cinereus, BRIss Or mith. VI. p. 201. Mergus Merganerer, Linn. Geil 63. 2.

952. MImbrian, Burr. XV. p. 401 pl XXII. Mergus mujor uxums, Bruss. Oinith. V. p. 120. pl. XI. 2. Colyuluns glucidis, Lixw. Cen.7 $7 \cdot 3 \cdot$

95.3. Femeile de linrle planche $25 \mathrm{I}$.

954. Canard . . . Buff. .... Cannrd a longue queue, Bruss. Ormith. V. p. 360. 1!. XXTV. 1. Anus acuta Linv. Gen 67. 28.

955. Canard Jenfin, Burr. . . . Anus Caterntefcens, (3!. Lins. Gen. 67. I 2 ? Oye fauvage de la Baye d'ludfon, Briss. Ornithe Vl. F. 275? (i).

956. Canard, granic macreufe, Burf. . . Anus fusca Linn. Gen. 67. 6. Ainls mure major, Briss. Omith. VI. p. 423 .

957. Le Pelican brum, Buff. XVI. p. 37. Oizoctetulus fluscuts, Briss. Oimith. VI. p 524. Pelecrnus onocrotulus occidentules, Linv. Gen. 72. I. (i).

958. Camad Chipeau, Burr. . Bkiss. Ornith. VI. p. 3.39 pl. XXY.IIf. I. Anus firepera, Liw. Gen. 67. 20 .

957. Anhinga, Burf. XVI. p. 253 pl. XI. Bruss. Ormith。 VI. p. 476. I\%otus anhinigu, Live. Gin. 73 .

960. Le même.

96r. Fregatte, Burf. XVI. p I 52. pl. VI. Fiegat Briss. Ornith. VI.p. 506. Pelcianus Aquilus, Liñ. Ger. 72.2. 
Pl.

\section{$(5 \ddot{z})$}

962. Petrel, Burf...... Puffumus Capitis bone Jpei, Briss. Ornith. VI. p. 137. Procellaria acquanoctialis, Linn. Gen. 70. 4 .

963. Albatros de la Chine, Buff. . . Briss. Ornith. VI. Linn. Gen. 70. o. 964. Petrel, Burr. .. Danicr Briss. Ornith. VI. p. 146. Procellaria Cas penfis, Linv. Gen. 70. 5 .

965. Varieté du Pelican je ne le trouve point cité chez M. Bufron.

966. Canard Soncroucou, Buff. . . Sarcelle d'Amerique, Briss. Ornith. VI. p. 453. Anuts discors Linn. Gen. 67. 37.

967. Cunard farcelle d'Amerique, Buff. .. Sarcelle d'Amerique, $\beta$. Briss. Ormeth. VI. p. 458 ? Anas discors. Linn. Gen. 67-37. $\beta$ ?

968. Camard (Sarcelle), Buff.... Sarcelle de St. Domingo, Briss. Or mith. VI. p. 472. fig. 2. Anas Dominica, Linn. Gen.67.22.

969. Petite Mouette cendrée, Burf. XVI. p. 227. Gavia cmerea major, BRIss. Ornith. VI. p. 178. pl. XVII. I. Larus cinerarius, LinN. 76.4 .

970. Mouette rieufe, Buff. XVI. p. 232. Gavia ridibunda, BRIss. Or. nith. VI. p. I92. pl. XVIII. I. Larus atricilla, Linv. Gen. 76. 8.

971. Canard Souchet, Buff. . . Briss. Ornith. VI. p. 329. 6. pl. XXXII. I. Anas clypeata, Linn. Gerı. 6. I9.

972. Camard fouchet femclle.

973. Petit Fou, Butf. XVI. p. I 42. Briss. Ornith. VI. O. Linn. Gen. 72. O. Pelecanus Leucogafter, mili.

974. Perit Foll brun, Burf. XVI. p. I 43. le fou brim, Briss. Ornith. p. 499. pl. Xlill. 1. Pelicanus filer, Linn. Gen. 72. 8.

975. Pingoin, Burf.... Stheniscus Briss. Ornith. VI. Diomedea demerfa, Lin. Gen. 7 I. .

976. Grand Courlis de Cayenne, Burr. XV. p. $75^{\circ}$ Briss. Graith. V. o. Scolopur caudatus mihi. Linn. Gen. 87. 0.

977. Moutte a pieds blens, Buff. XVI. p. Il 6. Guvia cinerea major, Briss. Ornith. VI. p. isz. Linn. Gen. 76. o.

978. Canmed Mncreufe, BurF. . . BRIss. Ornith. VI. pl. XXXVIII. 2. Alats nigra, Linn. Gen. 67.7.

979. Paille en queue a brins rouges, Buff. XVI. p. I16. Lepturus Briss. Omith.Vl. p. 409. Phaeton rubricauda, mihi. Lins. Gen. 74. o.

980. Camad (beau) hupé, Buff. ... Anas aefíva, Briss. Ornith. VI. p. 351. pl. XXXII. 2. Anas Jponja, Linn. Gen. 67. 43 .

981. Sa Femelle.

982. Oye ....... Buff. .... Oye d'Egypte, Briss. Ornith. VI. p. . 284 . pl. XVí. Ailis Egyptiaca, Linv. Gen. 67. IG.

98.3 . Sa Femelle.

984. Pingoin Manchot, Buff. . . . . . Briss. Ornith. VI. O. LinN. Gell. o.

985. Cannd, Oye funvage, Buff. ... Briss. Ornith. VI. p. 265. Anas anfer, Linv. Gell. $6 \%$.

986. Le fou racheré, Butr. XVI. p. I 44. Briss. Omith. VI. p. 497. Lins. Gcir.73.0. Pelecanus Sula.

987 . Picite Garin, Burf. XVI. p. 75. pl. III. Sterna major, Briss. Ornith. VI. 1. 203 . pl. XIX. I. Steria Hirmindo, LiNn. 77. 2. 
Pl

988. Grande Hirondelle de Mer de Caysenne, Bufr. XVI. p. Ico. BrIss. Oruth. o. Liñvi: Gètr. 77. o. Sterina maxima; royez Linn. n. 4. Steria minita.

989. Cahard musqué, BefF. V. Briss. Orinith. VI. p. 312. Anas mofclua. ta, Linn. Gen. 67. I6.

990. Goëland a mantèu noir; BUFr. XVI: p. I 87. pl. VII. Lairus Briss. Ornth. VI. p. 258. Larus marinus, Lins. 76. 6.

991. Labbe, Bufr. XVI. p. 243. pl. X. le ftercoraire, Bisss. Omith. VI. p. 150. Lamus parafiticus, Linn. Gem. 76. 10.

992. Petit Plongeon, Bur. XV. p. 394. Mergus minor, Briss. Omitit. VI. p. 108. Colymlats lmmer, Linn. Gen. 75.6.

993. Petrel. .... Burr.... Procellaria, Raiss. Omith. VI. p. i s.o. pl. XIII. x. Pracellaria pelagica, Linn. Gen: тo. J.

994. Mottette blanclic, Burf. XVI. p. 215: Briss. Ornith. VI. o. Larus nivens, mihi. Linn. Gen. 77.0.

995. Canard . . . Burr. . . . B Briss. Omith. VI. 0. Linn. Gen. 67. o. Anas latitoftris milhi.

996. Petire Hirondelle de Mer, Burr. XVI. p. 84. Sterna winor, Briss. Oimiti. VI. p. 106. Sterna migra, Livv. Gen. $7 \%$. 3.

997. Noddi, Buff. XVI. p. 272. pl. XIlI. Gavia fusca, Briss. Ornith. VI. p. 199. Sterna Jiolida, LinN. Gen. 77. 1.

998. Le Grand Paille en querce, Burr. XVI. p. III. Leptarus Briss. Ornit/. VI. p. 489. pl XXIV. I: Phaton athereus, Linn. Gen. 74. 1.

999. Canard Oedel, Burf. ... Sarcelle de l'Ine de Ferroé, Briss. Omith. VI. p. 40. pl. XL. 2. Lins. Gen.67.0.

1000. Canard ..... Burf. .... Sarcelle a tête rouge, Briss. Ornitt. VI. p. 435. A: Linv. Gen. 67. 0.

I001. Canard ..... Bufr..... petit MTorillon, Briss. Ornith. VI. p. IlI. pl. XXVI. 1. Lann. Gen. 67. 26. Anas Glaucion, (B).

1002. Canard ..... Buff. . . . . Millouin ( $\beta$ ). Briss. Ornith. VI. p. 384. pl. XXXV. 1. Anas ferima, Linn. Gen. 67. 3r.

1003. Le Pingoin, Burf. .... Uria minor, BRIss. Ornith. VI. p. 73. Alia Alle, Linn. Gen. 69. 5.

1004. Femelle du Pinǵoin, Buff. . . BRiss. Ornitr. VI. o. Lunv. Gen。 69. 0.

I005. Manchot des Hottentots, Burf. . . Briss. Ornith. VI. o. Linn. o. 1006. Oye des Terres Mugellaniques, Buff. . . . Briss. Ornith. VI. o. Lins. Gen. 67. 0.

100\%. Canard brun, Burf. ... Sarcelle de la Caroline. Briss. Ornith. VI. 39. Larns. Gell. 67. o. Anas notata, mihi.

1003. Canard de Miclon, Buff. . . B Buss. Ornuth. VI. o. Linn. Gen. 67. o. Anas miclonia, mihi.

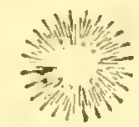




\title{
$\mathbb{T}$ A $\mathbb{B}$ \\ $\mathbb{L}$ \\ E
}

\section{A L P H A E T I Q UE}

\author{
DES OISEAUX REPRESENTÉS
}

D A N S L E S

PLANCHES ENLUMINÉES; SELON

LE S DENOMINATIONS.

D E

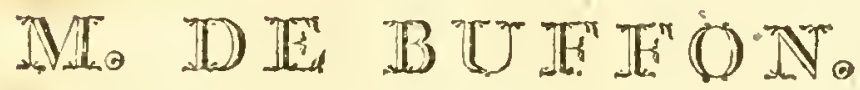

A.

$\Lambda$ gami.

Agripenne Or:olun.

Aigle Commun.

- de Pontichery.

- (grand)

- petit d'anerique.

Aigrette (Herost)

- rousfe.

Alatli (Marimpecheur)

Aibatros.

- - de la Chine.

Alcyon (Marbispech.)

Alipi.

Alouette.

- $\ldots$ noire.

- ado have. 738.1\%

Amazone Jaune (Perroq.) 13.

Auhinga. $\quad 10 ?^{\circ}$

Ani des Paletuviers. 102. 2.

- des Savannes. $\quad 102.1$.

Aonirou conarou (Perrog.) 547:

Apulé Juba (Perrog.) 528.

Ara rouge. $\quad 12.6+1$.

Ara verd. $\quad 383$.

- bleu.

- perruche.

36.

Arada.

864.

- 706

Autour (petit) de Cayenne. 473.

Autour. $\quad 413.461$.

- - blanc. $\quad 428$.

Autruche. $\quad 455^{\circ}$

Avolette. $\quad 35.5$

Azur petit. .060.

Azurin.

3.5.
B.

Balbufard. ' $\quad 414$.

Balicasfe. $\quad 603$.

Baltimote.

Bambe - 5 505.2.

Barbicall. 703 .

602.

430: 1. 2:

- $33^{1 .}$

- (grand) -371 .

- petit $\quad \therefore \quad 7+2.2$.

- a plaftron noir. 308.2 .

Barbll vert. $\quad 370$.

Barge commune: : 874 .

— iboyeufe. $\quad 876$.

— brune. $\quad 875$.

bartavelle. 900.

231.

Bec d'arcent: : : $\quad$ I50.

- en cifeall. : $\quad 218$.

Bícardé. - 296.

- inale: $304.3 \mathbf{I}$.

Becardé vanga. $\quad 304 \cdot 31 \%$

Bec figie. 668.1

Bec ouvert. $\quad 932$.

Becasfe. 885.

- des Sarannes. 895.

Becasfine du Cap. - 270.

- pe:ite. $\quad 883$

de madagascar. $\quad 022$.

Befroi (gland). 706.1.

Bengali. $\quad 258.115 .1$.

\begin{tabular}{ll}
- brun. & 115.2. \\
\hline piquet. & 115.3 .3$.
\end{tabular} 
Bentaveo.

Bergeroninette Janne.

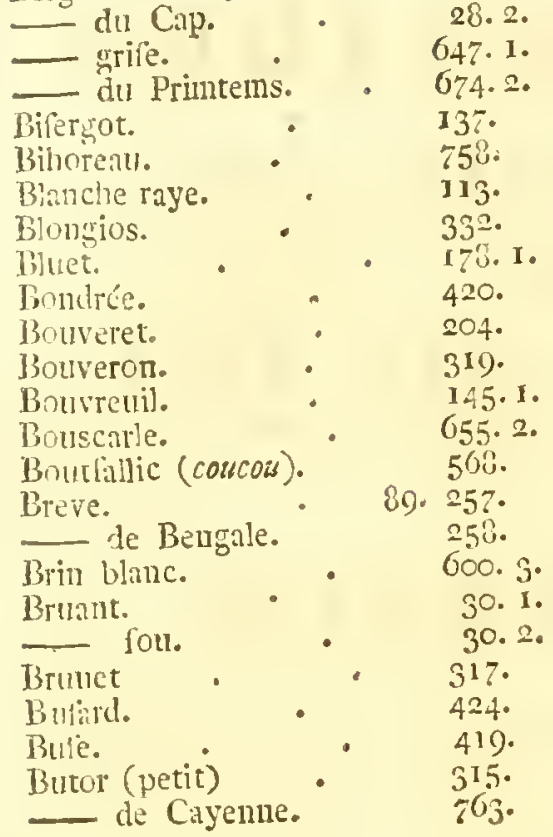

C.

Cabaret, a bec couleur de

$$
\text { Chair. }
$$

_- a huppe blanche.

191.

- a buppe rouge.

Caica (perruche).

Caille. des Isles inaloüues.

493.

749.

170.

C2]andre.

222.

302.2.

Cili crilic.

293.

Calno des Molucces. 283.

Calabystinie. $\quad 750$.

- de Malabar. . 8.3.

_ des Mlanilles. 891.

- Rhinoceros. - 934.

___ de l'ifle de Panay male. 780.

— Toc. - temelle. $73 \mathbf{1}$

__ 2 easque rond. $953^{\circ}$

Canard cravant. - 342.

_... Sarcelle de Louifanie. 403.

- de Marngnan. $80 \%$.

- farrage (inale). $\quad 7: 6$.

- de Milon. femelle.

- brun. $\quad 100 \%$

- (beau) huppé. 930.

— bec large" $\quad 995$.

Cannepetiere male. 25.

- n... femelle. 10.

Capmore. de St. Domingo 375.

(lein.) .5 .2 .
- - 535. 1. - olire de la Louifanie. 60\%. 2. Cardinal huppé.

Can dominicain huppé. 103.

Catrillouneur. $\quad$ 702.2.

Casfenoix. 50.

Casfenoilette. - 302.1.303.1.

Castican. 628.

Castique. 64.

\begin{tabular}{ll} 
- \\
- huppe. & 344. \\
\hline & 323.
\end{tabular}

Casque noir. $\quad 392$.

Cafoar. $\quad 313$.

Castingueur des Philippines. 945 .

Catraguenx. $\quad 505$.

Caudec Gobemouche. $\quad 453.2$.

Calurale. $\quad z v_{4}$

Ceinture de pretre. . 650.2.

Chalybé de la N. Guinće. 634.

Charbonmiere. $\quad 3 . \mathrm{I}$.

Chardonneret. $\quad 4.1$.

- - - blanc. 4.2.

Chardonneret Jaume. $\quad 202.2$.

Chathuant. $\quad 43 ?^{\circ}$

- - - de Cayenne. 442.

Cheveclie. $\quad 4.39$.

Chevalier varié. $\quad .300$.

- - raye. 484.

- - - commun.

- - a pieds rouges. 845.

Chonette. $\quad 43^{3}$.

- - - a longue queure. 463 .

Cholicas. 52.30

- - chauve. $\quad 521$.

- - monfache. 226.

- - de la Now. Guin. 629.

Choucari. $\quad 630$.

Chouk. . . 522 .

Clioguard. $\quad 53$.

Cicoone. $\quad 566$.

Cincle. noire. $\quad 399$.

Coalic. $\quad 577 \cdot 729$.

Cocheris. 500.1

Locq. die bruiere.

—_ huppé. $\quad$ 75. 74.

— de roche. 39.40.

Con de Perl. 745 .

Cocotzin (Pigeon). $24.5^{\circ}$

( oilfe noire. 720.2 .736 .

Coifie blanche. - 35.3 .

Col nud. $\quad 609$.

Colibri topaze. $\quad 599.1$.

- violet. $\quad-\quad$.

- - petit. $\quad 600 . \mathbf{1 .}$

- a collier rouse. $\quad(100.4$.

- a cravatte verte. 671.4.

- amethylte. 672. I.

- crivarte dorée. - 5.

Coliou a quelle violete. 1 .

-

Colma. $\quad 703.1$. 


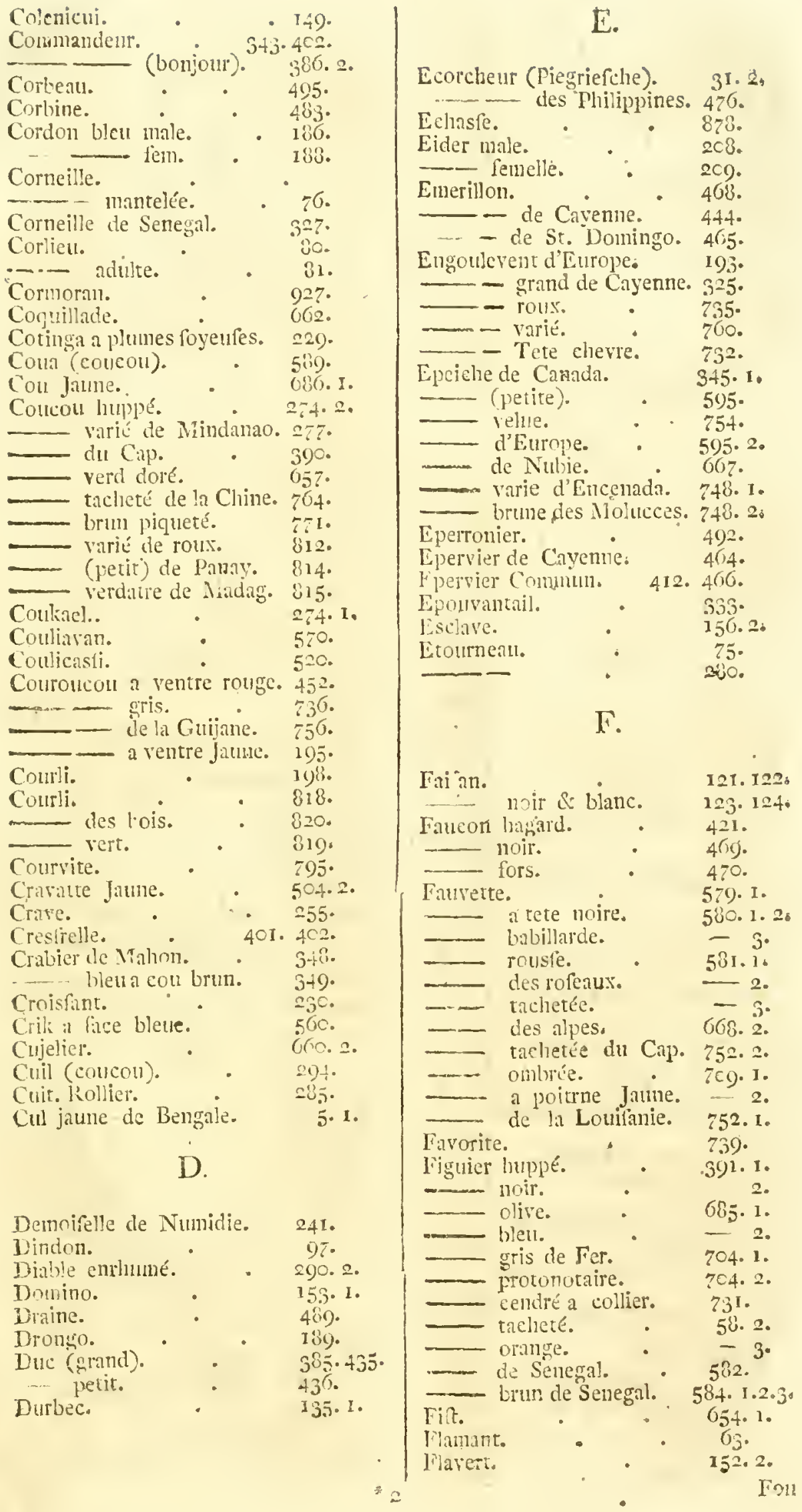




\begin{tabular}{|c|c|c|}
\hline Troul (petit). & & \\
\hline $\begin{array}{l}\text { lou (petic). } \\
\text { - brun. }\end{array}$ & & $\begin{array}{l}030 \\
0.4\end{array}$ \\
\hline Fou de basfan. & - & 978. \\
\hline Fou taclietée. & - & 936. \\
\hline Foudi (lriquet). & - & 6. I. \\
\hline Foulque. & • & $\begin{array}{l}134.10 \\
197 .\end{array}$ \\
\hline Foulque a crete. & • & 797. \\
\hline Founingo (Pigeon). & & 11. \\
\hline $\begin{array}{l}\text { blell. } \\
\text { Fourinilier a oreille }\end{array}$ & - & III. \\
\hline $\begin{array}{l}\text { blanches. } \\
\text { bintion }\end{array}$ & & 322.1 .2$. \\
\hline & - & $823 \cdot 2$ \\
\hline $\begin{array}{l}\text { Frefaye. } \\
\text { Treux. }\end{array}$ & - & 140. \\
\hline $\begin{array}{l}\text { lreux. } \\
\text { Iriquet. }\end{array}$ & • & \\
\hline - huppé. & - & 131.1. \\
\hline
\end{tabular}

\section{G.}

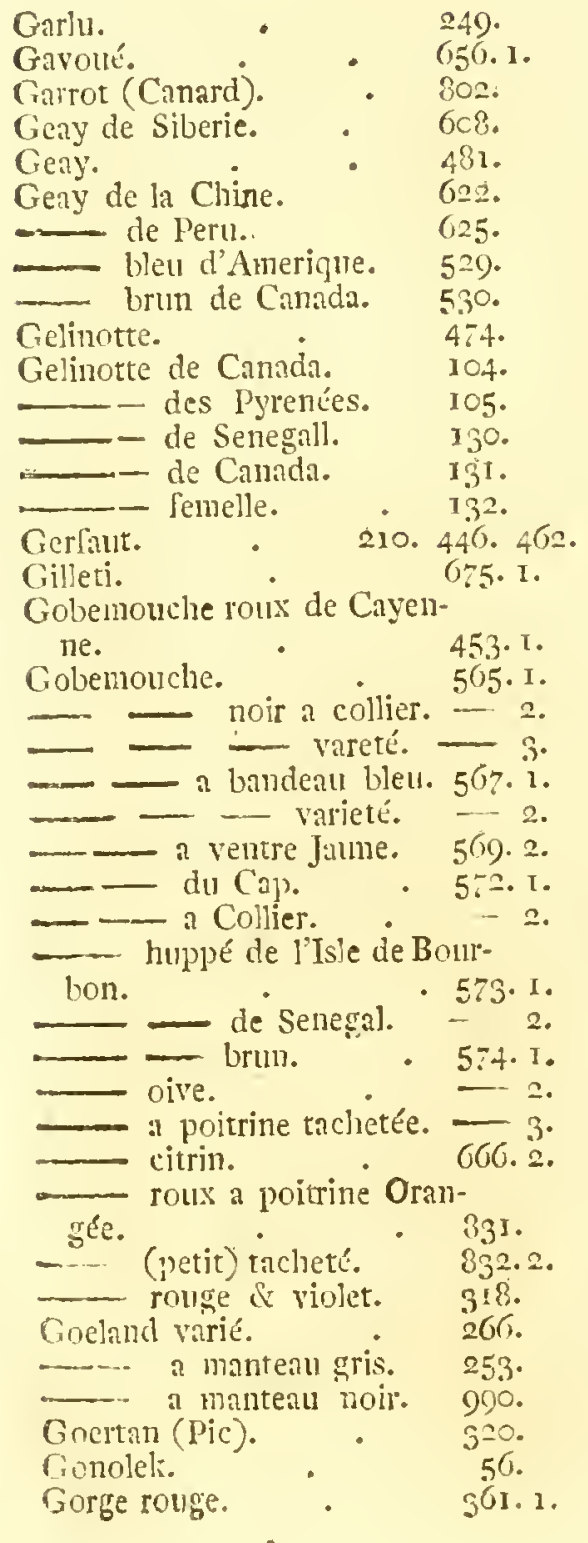

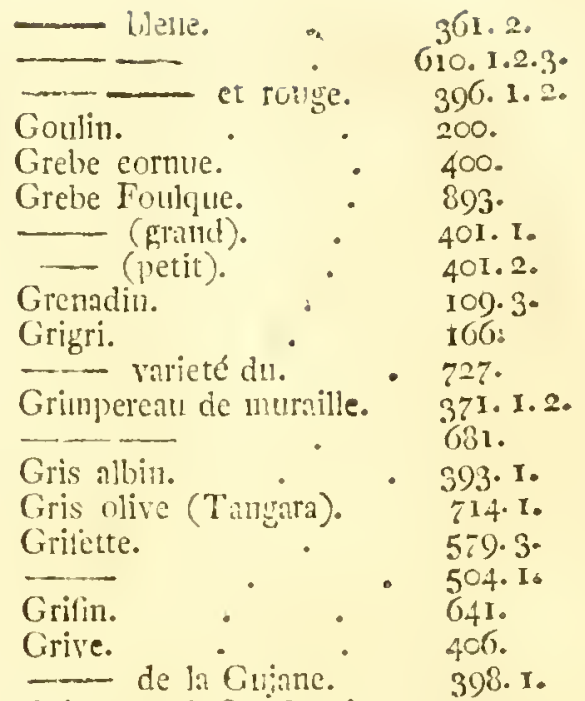

Grivelette de St. Domingo. 398. 2.

Grivelin. $\quad$ - $\$ 09$.

Givert. • (316.

Grosber tacheté du Cap. 659. I.

- d'Angola. . - 2.

- 99. I00.

-

- bleu d'Ainerique. $\quad \mathbf{5} 4$.

Give. $\quad$ - 760.

Guariuba.
Guepier verd a queue d'azur. 525 .

- marron et b?eu. $\quad 252$.

- verd a gorge bleue. 740 .

Guerarou. • 609.

Guillemot. $\quad 850$.

Guipanga.

Guinguette. $\quad$ - 350:

Guernegat. $\quad 321.1$.

Guitguit vert \& bleu a tete bleue.

-.... _ a tete noire. $\quad-\quad 2$.

- 682. I.

- - - Ealieté. - 2 .

\section{H.}

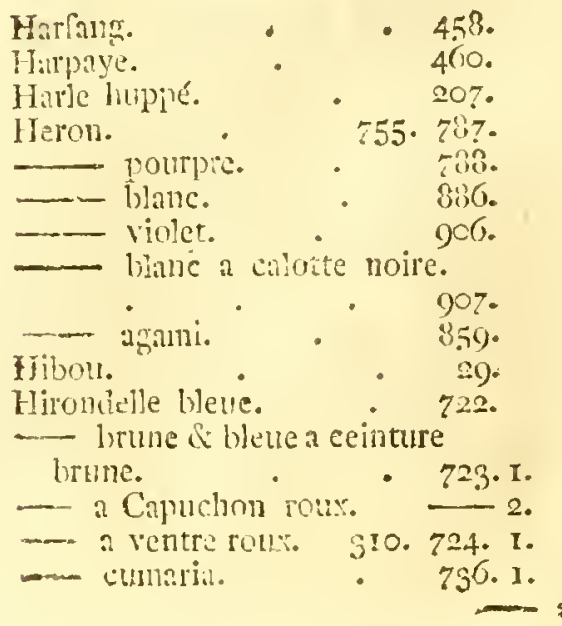




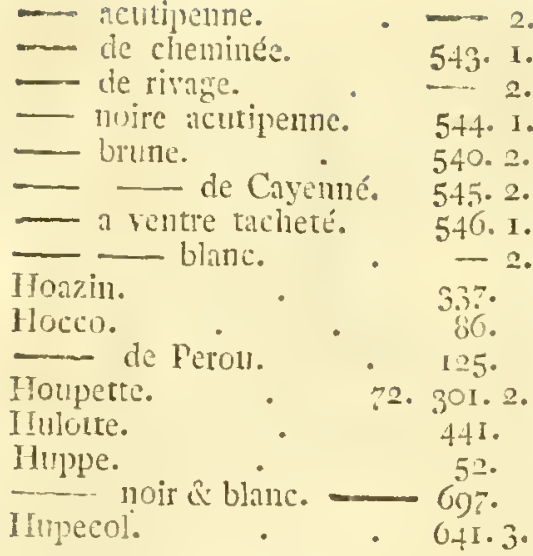

\section{J.}

Tabiru.

iacarini.

•

- le male. $\quad$ - 2.

- varieté. $\quad$ femclle. $\quad 3$

lacamare. $\quad$ - 2.53.

a longue quene. $27 \tau$.

acarili. $\quad 22$.

lacana. $\quad 316$.

facou. 133.1

fiaco (Pertoquet). 31t.

lasuacati. 503 .

Jinsent. $\quad 261$.

lasmoir (Merle). $\quad 199$.

fbitiu.

lean le blanc. $\quad 41 \mathrm{ij}$

fetis. $\quad 380$.

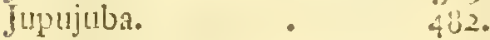

. $\mathrm{k}$.

Katraca (Rifin). $\quad 146$

Kanichy. $45 \mathrm{I}$.

Kinki manof (Gobenf. 54 .

Kiolo (rale) . 368.

Kiolo. . . 753

inutgechef. $\quad 30 \%$

\section{L.}

Talue.

Isangrayien.

Lagopede.

Lavandiere.

Linotte.

Liturne.

.... de Canada.

J ori a collier.

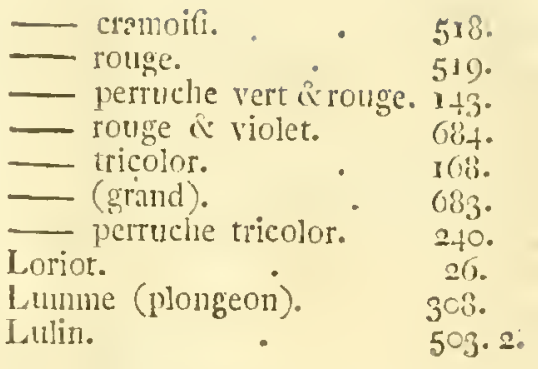

\section{M.}

Mainate. $\quad 2 \quad 263$

Majan. $\quad$ ICj. 1.

\begin{tabular}{l} 
Nacarcux. \\
Manaquin a tete d'or. \\
\hline
\end{tabular}

- -... blanche. - 2 .

- rotse. 3 - 3 .

- a grorge blanclis. 324. I.

- varić. -2

Vanicor. 0 302.2.

lanchot pingoin. 707.2.

- - des Hottentots. $\quad 984$.

Manncode a bouquet. $\quad 6_{3} \mathrm{I}$.

- noir. 632.

Marail. $\quad 496$.

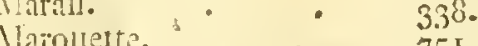

Martin pecheur a tete bleue $75 \mathrm{I}$.

350.

- - blen \& roux: 232.

- - vert 2 blanc. $59 i$.

- vert \& roux. 592.2,

- - a tete grie. 594.

- - (le pius grand). 683.

- - pic. 716.

- - a tete \& con couleur

de paille. . $\quad 757^{\circ}$

- - Orange. $\quad 757.2$.

- - roux. 782.

- - de liadigazcar. 7z8. 1.

- a tete verte. $\quad-33,2$.

- huppé. .679.

- a coilie noire. 673 .

- 62.

Iinain. : 219.

Martinet noil a ventie b]anc. 545 . 1 .

- a collier blanc. 725.2 .

$\begin{array}{ll}\text { petit. } & 725.1 \\ \text { Maubeclie tachetée. } & 365 .\end{array}$

Mascarin. grile. $\quad 260$.

Nifatuitui. : 884.

Merle. 2.

- hujppé de la Chine. 50 .

- a plaftron blanc. $\quad 516$.

- violet. 540.

- de Montagne. $\quad 182$.

- couleur de Role. $25 \mathrm{I}$.

- blell..$\quad 250$.

- olí 


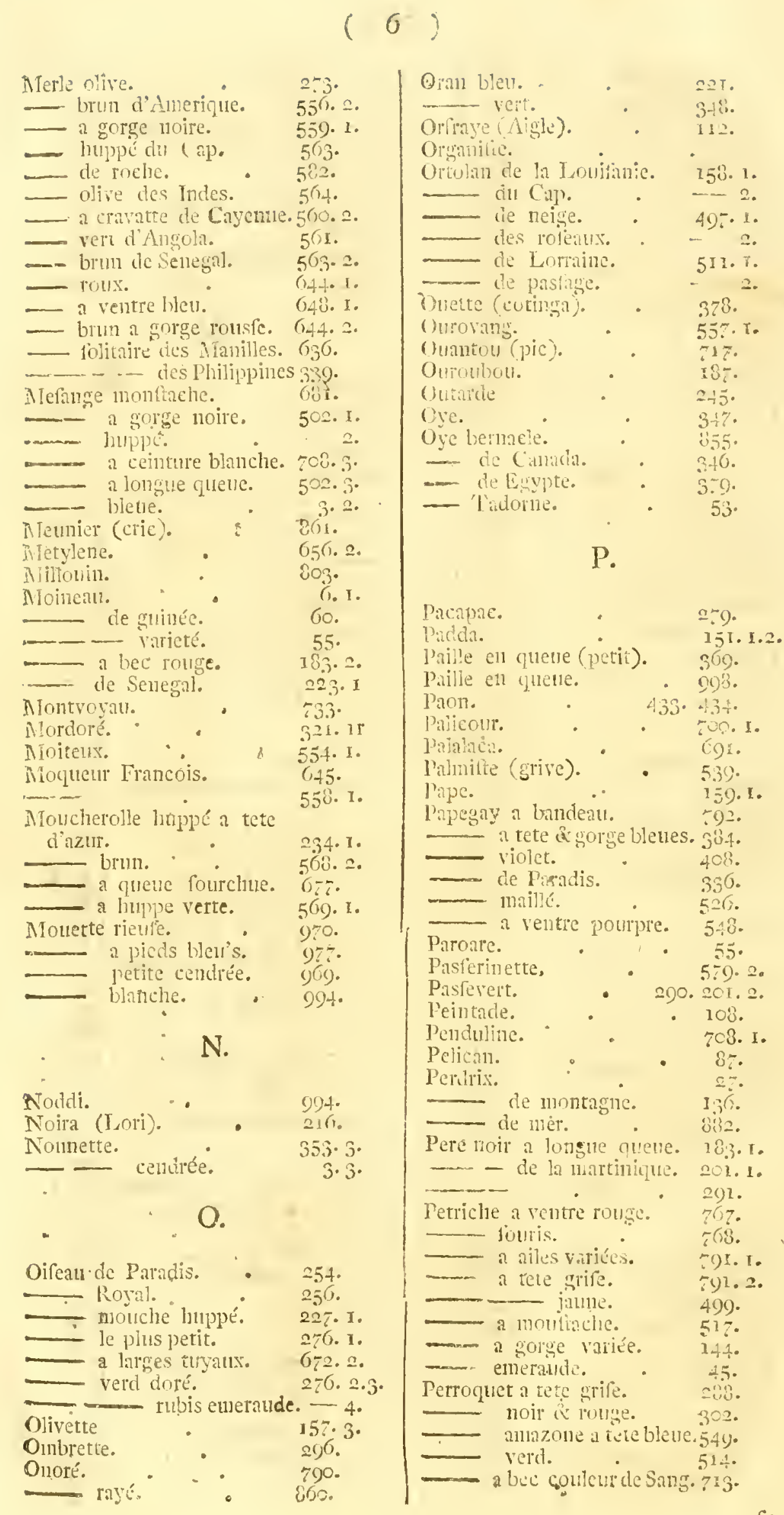




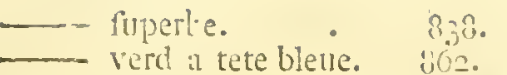
tapiri. 100 . Pernclie a tate blene. IDo. 2.61. -... a gorge verte. 144. Pcituche a tete bleve cinnene longth. 192. - in double Collier.

- a aiks ronges. $\quad 2.3 y$.

- a tete romge. 264.

- - a ailes chamarrées. 207.

-ma a collier rouge vif. 6.t2.

_ a tete couleur de rofe

a longs brins. $\quad 808$.

- (grande) a longs brins

Phaifan bicolor.

$88 \%$

Jhalarepe.

l'ic noir a domino rouge. i1..

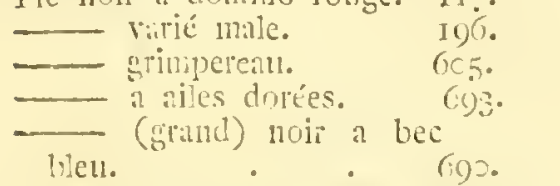

- roux $\quad 00 \div 1$.

man- (petit) noir. - 2

- no noir a huppe rouge. 713.

-... (grand) ralyé de Ciyen-

ne. rerd de Bengale. To. dis Goo $095^{\circ}$

- Virié de la Carolinc. zúj.

ne. (tres petit) de Cayer-

ne. a tete grife. $\quad 586.2$

- a cravate noire. 863.

- (petio) rayé de sene-

תia

n- vert. $\quad 3$.

-

_- varié de Jamaique. 59:-

-..... Jaune di Cayenne. 5cy.

- raye de St. Noningo. $28 \mathrm{I}$

- rordoré. • 524.

_... virić ondé. (1) 553.

mingo. 6 It

ne. de Cayen 6r.s.

- verd rayé. $\quad 379$

- a col rouge.

Pic. ... 43̈4.

- de Senegal. 538 .

Piegriefithe grite. 445

- - variée. $\quad$ - $2.47 \%$. I.

_- rousfe du Cap. - 2.

___ bleue de Madigascar.s93. I.

_- de Sellegal. $29 \% .1$.

Dierre gincúc.

yietre mile- . 4 479.

pigeon komain. . 110. rertales Mhilipyines. 198 .

- commum. $407^{\circ}$

- de Martinique. IfI.

- ne Nicobar. $49 \mathrm{I}$.

$\longrightarrow$ de Banclia. 118.

- ramier des Nolucces. I64.

Pingoin. 3\% 30. 975. 1003.

Pipi. . 66r. 2.

- bleu. 669.1 .20

pique boenl. $=3$.

Pitchou. 655.

Pivotte Ortolane. . 652. .

Plafiton noir. . 277.

- blanc. de Cayeme. 630. 1.

Pinvier dorć.

- guignard. . 332.

-

- coiftè. $\quad 334$.

omme a lambenux. $\quad 380$

- colronné. $\quad 300$.

- - a aistettes. 801

- - a collier. . $\quad 286$.

Plongeon (grand). $\quad 914$.

- (petit) . 992.

Pjumet blanc (manalin). 70\%. I.

podobé. . 354.

pouilyt. : 65I.

Poule de Inpan. $\quad 98$.

-.. duliane. 0.00.

- - brune. 396.

$\ldots$ ben (srande). 352.

- 877.

Proyer.

1'romerojs a ventre tacheté

a parements 638.

prefirgule. . 411.

Q.

Quadricolor. -101.2

Qucreibi. . . 6 64

Quene en Eventiil. 380.

H.

Lille d'cat.

749.

- de genet. 550.

- tacheté de Cayenne. 775 .

- (petir) de Cayeune. 847.

- a long bec. 849.

Ramier. $\quad 316$.

Ramiret. 213 .

Remiz. $\quad \therefore \quad 618$.

Rochier . $\quad 447$ 。

Jioi des Gobenonches. 293.

Roirelet, . $\quad 708.2$.

730.1.2.

Rol- 


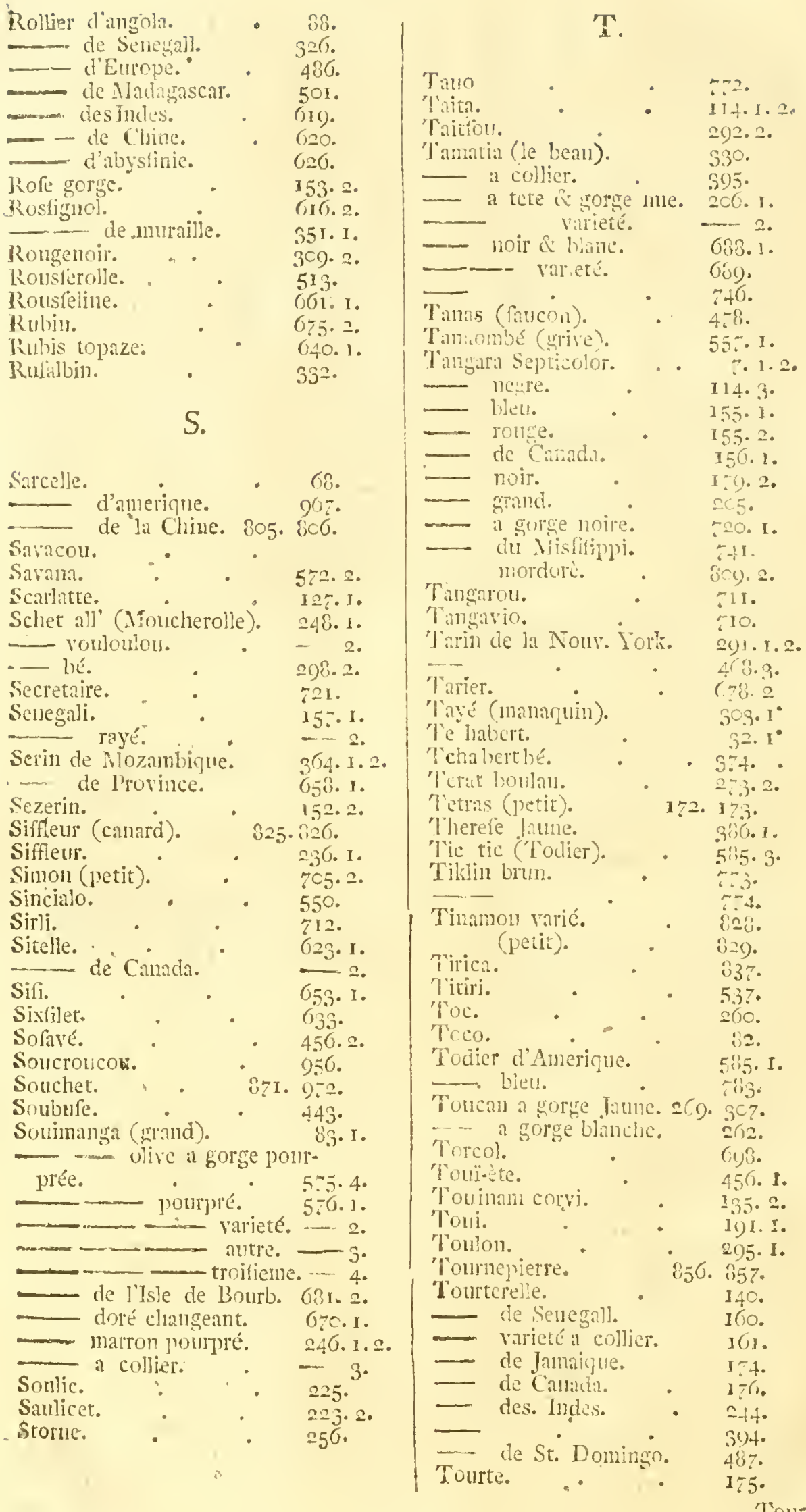




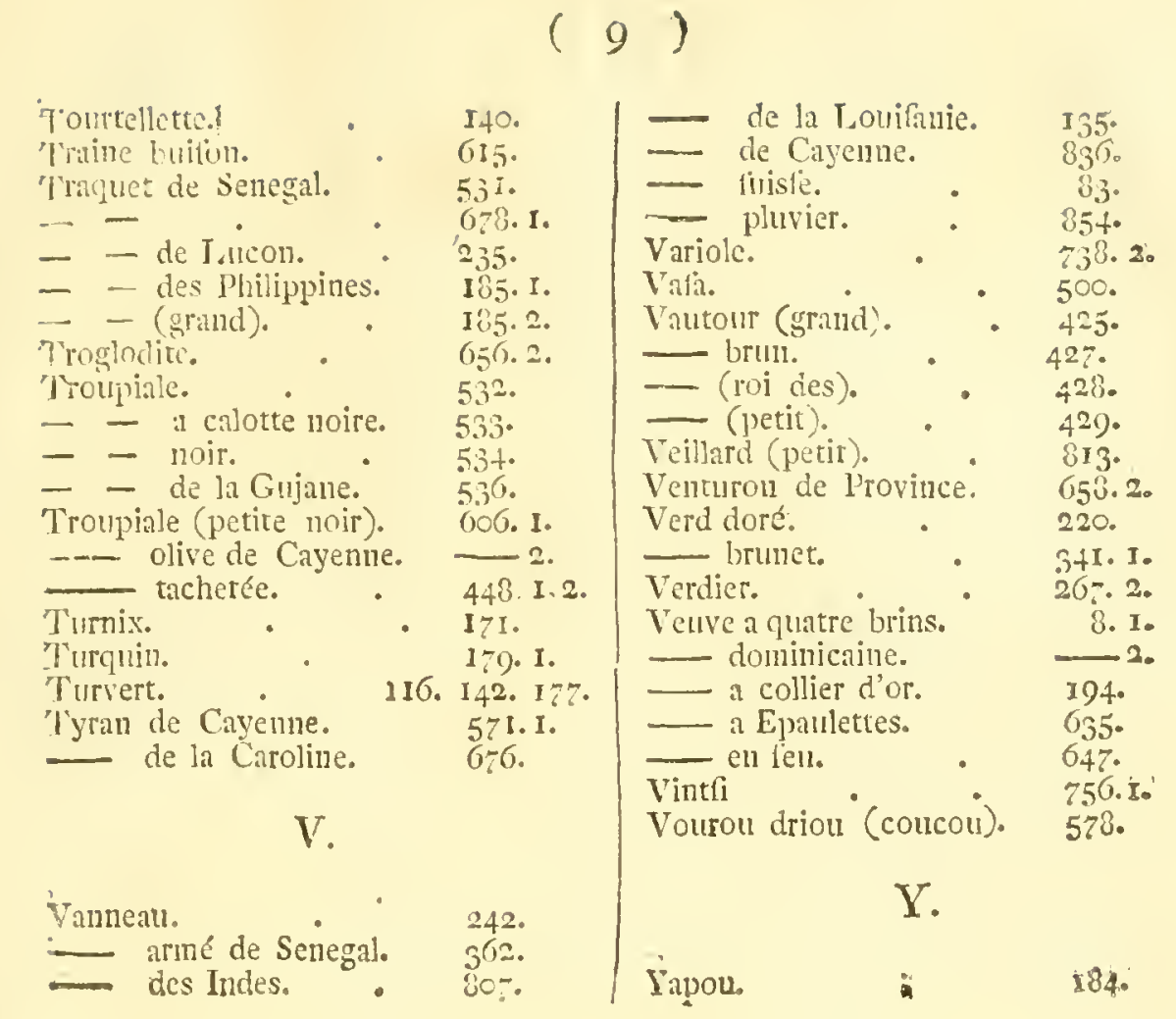

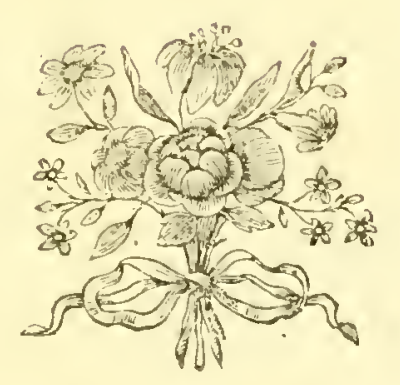








